\title{
A search for the standard model Higgs boson decaying to charm quarks
}

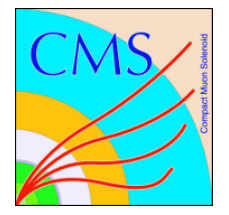

\section{The CMS collaboration}

E-mail: cms-publication-committee-chair@cern.ch

ABSTRACT: A direct search for the standard model Higgs boson, H, produced in association with a vector boson, $\mathrm{V}$ ( $\mathrm{W}$ or $\mathrm{Z}$ ), and decaying to a charm quark pair is presented. The search uses a data set of proton-proton collisions corresponding to an integrated luminosity of $35.9 \mathrm{fb}^{-1}$, collected by the CMS experiment at the LHC in 2016, at a centre-of-mass energy of $13 \mathrm{TeV}$. The search is carried out in mutually exclusive channels targeting specific decays of the vector bosons: $\mathrm{W} \rightarrow \ell \nu, \mathrm{Z} \rightarrow \ell \ell$, and $\mathrm{Z} \rightarrow \nu \nu$, where $\ell$ is an electron or a muon. To fully exploit the topology of the $\mathrm{H}$ boson decay, two strategies are followed. In the first one, targeting lower vector boson transverse momentum, the $\mathrm{H}$ boson candidate is reconstructed via two resolved jets arising from the two charm quarks from the $\mathrm{H}$ boson decay. A second strategy identifies the case where the two charm quark jets from the $\mathrm{H}$ boson decay merge to form a single jet, which generally only occurs when the vector boson has higher transverse momentum. Both strategies make use of novel methods for charm jet identification, while jet substructure techniques are also exploited to suppress the background in the merged-jet topology. The two analyses are combined to yield a $95 \%$ confidence level observed (expected) upper limit on the cross section $\sigma(\mathrm{VH}) \mathcal{B}(\mathrm{H} \rightarrow \mathrm{c} \overline{\mathrm{c}})$ of $4.5\left(2.4_{-0.7}^{+1.0}\right) \mathrm{pb}$, corresponding to 70 (37) times the standard model prediction.

KEYwords: Hadron-Hadron scattering (experiments), Higgs physics, Charm physics

ARXIV EPRINT: 1912.01662 


\section{Contents}

1 Introduction 1

2 The CMS detector 3

3 Simulated event samples $\quad 4$

4 Event reconstruction and selection $\quad 5$

$\begin{array}{ll}4.1 \text { Baseline selection } & 6\end{array}$

5 Resolved-jet topology analysis $\quad 8$

5.1 Higgs boson reconstruction 8

$\begin{array}{lll}5.2 \text { Signal extraction } & 10\end{array}$

6 Merged-jet topology analysis $\quad 12$

$\begin{array}{lll}6.1 & \text { Higgs boson reconstruction } & 12\end{array}$

$\begin{array}{ll}6.2 \text { Signal extraction } & 14\end{array}$

$\begin{array}{llr}7 & \text { Systematic uncertainties } & 18\end{array}$

$\begin{array}{llr}8 & \text { Results } & 19\end{array}$

8.1 Resolved-jet topology 21

8.2 Merged-jet topology 21

$\begin{array}{ll}8.3 \text { Combination } & 24\end{array}$

$\begin{array}{lll}9 & \text { Summary } & 25\end{array}$

$\begin{array}{ll}\text { The CMS collaboration } & 34\end{array}$

\section{Introduction}

The discovery of a Higgs boson, H, with the CERN LHC data collected in 2010-2012 by both the ATLAS [1] and CMS [2,3] experiments in 2012 represented a major step toward the characterisation of the electroweak symmetry breaking mechanism [4-6]. The mass of this particle is measured to be $m_{\mathrm{H}} \sim 125 \mathrm{GeV}[7-9]$ and its decays in the $\gamma \gamma, \mathrm{ZZ}, \mathrm{WW}$, and $\tau \tau$ modes have been observed [10-20]. All measured properties so far [7, 8, 8, 21-29] indicate that, within the measurement uncertainties, this new particle is consistent with the expectations of the standard model (SM). Nevertheless, there remains much to be learned about the properties of this new particle. One of the highest priorities of the LHC physics program is the measurement of the couplings of the $\mathrm{H}$ boson to other SM particles. Recently both ATLAS and CMS Collaborations reported the first direct measurements of 
the $\mathrm{H}$ boson couplings to third-generation quarks ( $\mathrm{t}$ and $\mathrm{b}$ ) [30-33] and found them to be also compatible with the SM prediction. A measurement of the couplings of the $\mathrm{H}$ boson to second generation leptons $[34,35]$ and quarks is the next target.

In this paper, we focus on the search for $\mathrm{H}$ bosons decaying to $c \overline{\mathrm{c}}$, a charm quarkantiquark pair. The $\mathrm{H}$ boson to charm quark Yukawa coupling $y_{\mathrm{c}}$ can be significantly modified by physics beyond the SM [36-40]. In the absence of an observation of Higgs decays to charm quarks, one can place a bound on the charm quark Yukawa coupling. The first direct bound on $\kappa_{\mathrm{c}} \equiv y_{\mathrm{c}} / y_{\mathrm{c}}^{\mathrm{SM}}$ of 234 at $95 \%$ confidence level (CL) has been obtained by recasting the ATLAS and CMS $8 \mathrm{TeV} \mathrm{H} \rightarrow \mathrm{b} \overline{\mathrm{b}}$ searches [41] in a model independent way. Indirect constraints on $y_{\mathrm{c}}$ obtained from a global fit to existing $\mathrm{H}$ boson data result in an upper bound on $\kappa_{\mathrm{c}} \equiv y_{\mathrm{c}} / y_{\mathrm{c}}^{\mathrm{SM}}$ of 6.2 [41] at $95 \%$ confidence level (CL), assuming the absence of non-SM production mechanisms. A direct measurement of this process is extremely challenging at a hadron collider. The branching fraction of this process according to $\mathrm{SM}$ computations, $\mathcal{B}(\mathrm{H} \rightarrow \mathrm{c} \overline{\mathrm{c}})=0.0288_{-0.0006}^{+0.0016}$ [42], is a factor 20 smaller than that of $\mathrm{H} \rightarrow \mathrm{b} \overline{\mathrm{b}}$, and there is a very large background from SM processes comprised uniquely of jets produced through the strong interaction, referred to as quantum chromodynamics (QCD) multijet events. Results from direct searches for $\mathrm{H} \rightarrow c \overline{\mathrm{c}}$ at the $\mathrm{LHC}$ in the $\mathrm{ZH} \quad(\mathrm{Z} \rightarrow \ell \ell$, $\ell=\mathrm{e}$ or $\mu$ ) channel were previously reported by the ATLAS Collaboration using a data sample of proton-proton (pp) collisions at a centre-of-mass energy of $13 \mathrm{TeV}$, corresponding to an integrated luminosity of $36.1 \mathrm{fb}^{-1}$ [43]. The observed (expected) exclusion limit on the signal strength $\mu$ (defined as the product of the measured $\mathrm{H}$ boson production cross section and the $\mathrm{H} \rightarrow \mathrm{c} \overline{\mathrm{c}}$ branching fraction divided by the same quantity as predicted by the SM) at 95\% CL was found to be 110 (150).

This paper presents the first direct search for the $\mathrm{H} \rightarrow \mathrm{c} \overline{\mathrm{c}}$ decay carried out by the CMS Collaboration. It uses pp collision data corresponding to an integrated luminosity of $35.9 \mathrm{fb}^{-1}$, collected with the CMS experiment at the LHC in 2016 at a centre-of-mass energy of $13 \mathrm{TeV}$. The search targets $\mathrm{H}$ bosons produced in association with a $\mathrm{W}$ or $\mathrm{Z}$ boson, which we collectively refer to as vector $(\mathrm{V})$ bosons. The presence of a $\mathrm{V}$ boson greatly suppresses backgrounds stemming from otherwise overwhelming QCD multijet processes, and its leptonic decays provide a crucial handle to collect the events efficiently. The most significant remaining backgrounds arise from $\mathrm{V}+$ jets (processes that account for one or more jets recoiling against a vector boson), $\mathrm{t} \overline{\mathrm{t}}$, and $\mathrm{VH}(\mathrm{H} \rightarrow \mathrm{b} \overline{\mathrm{b}})$ processes. To fully explore the $\mathrm{H} \rightarrow \mathrm{c} \overline{\mathrm{c}}$ decay mode, the analysis is split into two separate searches involving different topologies: the "resolved-jet" topology, in which the $\mathrm{H}$ boson candidate is reconstructed from two well-separated and individually resolved charm quark jets, and the "merged-jet" topology, in which the hadronisation products of the two charm quarks are reconstructed as a single jet. The former focuses on $\mathrm{H}$ boson candidates with lower transverse momentum, $p_{\mathrm{T}}$, while the latter performs better for $\mathrm{H}$ boson candidates with high $p_{\mathrm{T}}$. In practice, the two topologies can have significant overlap and so, for the final result, the two are made distinct by defining them in reference to whether the $\mathrm{V}$ boson in the event has $p_{\mathrm{T}}(\mathrm{V})$ below or above a single threshold chosen to maximise the sensitivity to the $\mathrm{VH}(\mathrm{H} \rightarrow \mathrm{c} \overline{\mathrm{c}})$ process. 
The central feature of this search is the identification of charm quark jets. In both topologies, novel tools based upon advanced machine learning (ML) techniques are used for charm quark jet identification [44, 45]. In addition, the merged-jet topology makes use of jet substructure information to further suppress the backgrounds.

\section{The CMS detector}

The central feature of the CMS apparatus is a superconducting solenoid of $6 \mathrm{~m}$ internal diameter, providing a magnetic field of $3.8 \mathrm{~T}$. Within the solenoid volume are a silicon pixel and strip tracker, a lead tungstate crystal electromagnetic calorimeter (ECAL), and a brass and scintillator hadron calorimeter (HCAL), each composed of a barrel and two endcap sections. Forward calorimeters extend the pseudorapidity $(\eta)$ coverage provided by the barrel and endcap detectors. Muons are detected in gas-ionisation chambers embedded in the steel flux-return yoke outside the solenoid.

In the barrel section of the ECAL, an energy resolution of about $1 \%$ is achieved for unconverted or late-converting photons that have energies in the range of tens of $\mathrm{GeV}$. The remaining barrel photons have a resolution of about $1.3 \%$ up to $|\eta|=1$, rising to about $2.5 \%$ at $|\eta|=1.4$. In the endcaps, the resolution of unconverted or late-converting photons is about $2.5 \%$, while other endcap photons have a resolution between 3 and $4 \%$ [46].

In the region $|\eta|<1.74$, the HCAL cells have widths of 0.087 in $\eta$ and $0.087 \mathrm{rad}$ in azimuth $(\phi)$. In the $\eta$ - $\phi$ plane, and for $|\eta|<1.48$, the HCAL cells map on to $5 \times 5$ arrays of ECAL crystals to form calorimeter towers projecting radially outwards from close to the nominal interaction point. For $|\eta|>1.74$, the coverage of the towers increases progressively to a maximum of 0.174 in $\Delta \eta$ and $\Delta \phi$. Within each tower, the energy deposits in ECAL and HCAL cells are summed to define the calorimeter tower energies.

Muons are measured in the range $|\eta|<2.4$, with detection planes made using three technologies: drift tubes, cathode strip chambers, and resistive-plate chambers. The efficiency to reconstruct and identify muons is greater than $96 \%$. Matching muons to tracks measured in the silicon tracker results in a relative $p_{\mathrm{T}}$ resolution, for muons with $p_{\mathrm{T}}$ up to $100 \mathrm{GeV}$, of $1 \%$ in the barrel and $3 \%$ in the endcaps. The $p_{\mathrm{T}}$ resolution in the barrel is better than $7 \%$ for muons with $p_{\mathrm{T}}$ up to $1 \mathrm{TeV}$ [47].

Events of interest are selected using a two-tiered trigger system [48]. The first level, composed of custom hardware processors, uses information from the calorimeters and muon detectors to select events at a rate of around $100 \mathrm{kHz}$ within a fixed time interval of less than $4 \mu \mathrm{s}$. The second level, known as the high-level trigger (HLT), consists of a farm of processors running a version of the full event reconstruction software optimised for fast processing, and reduces the event rate to around $1 \mathrm{kHz}$ before data storage.

A more detailed description of the CMS detector, together with a definition of the coordinate system used and the relevant kinematic variables, can be found in ref. [49]. 


\section{Simulated event samples}

Signal and background processes are simulated using various event generators, while the CMS detector response is modelled with GEant4 [50]. The quark-induced ZH and WH signal processes are generated at next-to-leading order (NLO) accuracy in QCD using the POWHEG v2 [51-53] event generator extended with the Multi-scale improved NLO (MiNLO) procedure [54, 55], while the gluon-induced $\mathrm{ZH}$ process is generated at leading order (LO) accuracy with POWHEG v2. The $\mathrm{H}$ boson mass is set to $125 \mathrm{GeV}$ for all signal samples. The production cross sections of the signal processes [42] are corrected as a function of $p_{\mathrm{T}}(\mathrm{V})$ to next-to-next-to-leading order (NNLO) QCD + NLO electroweak (EW) accuracy combining the vHNNLO [56-59], vH@NNLO [60, 61], and HAWK v2.0 [62] generators as described in ref. [42].

The V+jets events are generated with MADGRAPH5_aMC@NLO v2.4.2 [63] at NLO with up to two additional partons, and at LO accuracy with up to four additional partons. The production cross sections for the $\mathrm{V}+$ jets samples are scaled to the NNLO cross sections obtained using FEWZ 3.1 [64]. Events in both LO and NLO samples are reweighted to account for NLO EW corrections to $p_{\mathrm{T}}(\mathrm{V})$, which reach up to $10 \%$ for $p_{\mathrm{T}}(\mathrm{V}) \approx 400 \mathrm{GeV}$. In addition, a LO-to-NLO correction is applied to LO samples as a function of the separation in $\eta$ between the two leading jets in the event [65]. The $p_{\mathrm{T}}(\mathrm{V})$ spectrum in simulation after the aforementioned corrections is observed to be harder than in data, as expected due to missing higher-order EW and QCD contributions to the $\mathrm{V}+$ jets processes [66]. A residual reweighting of $p_{\mathrm{T}}(\mathrm{V})$, that is obtained via a fit to the data-to-simulation ratio in the control regions (detailed in section 5) of the $\mathrm{W}(\ell v) \mathrm{H}(\mathrm{c} \overline{\mathrm{c}})$ and $\mathrm{Z}(\ell \ell) \mathrm{H}(\mathrm{c} \overline{\mathrm{c}})$ channels in the resolved analysis, is applied.

Diboson (WW, WZ and ZZ) background events are generated with MADGrAPH5_aMC@NLO v2.4.2 [63] at NLO with up to two additional partons in the matrix element calculations. The same generator is used at LO accuracy to generate a sample of QCD multijet events. The $t \bar{t}[67]$ and single top production processes in the tW- and $t$ channels $[68,69]$ are generated to NLO accuracy with POWHEG v2, while the $s$-channel [70] single top process is generated with MADGRAPH5_aMC@NLO v2.4.2. The production cross sections for the $t \overline{\mathrm{t}}$ samples are scaled to the NNLO prediction with the next-to-next-to-

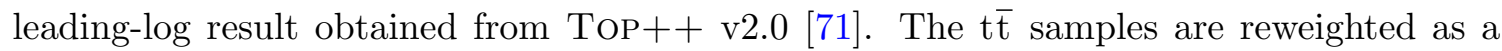
function of top quark $p_{\mathrm{T}}$ to account for the known differences between data and simulation [72].

The parton distribution functions (PDF) used to produce all samples are the NNLO NNPDF3.1 set [73]. For parton showering and hadronisation, including the $\mathrm{H} \rightarrow \mathrm{c} \overline{\mathrm{c}}$ decay, the matrix element generators are interfaced with PYTHIA v8.230 [74] with the CUETP8M1 [75] underlying event tune. The matching of jets from matrix element calculations and those from parton shower is done with the FxFx [76] (MLM [77]) prescription for NLO (LO) samples. For all samples, simulated additional pp interactions in the same or adjacent bunch crossings (pileup) are added to the hard-scattering process. The events are then reweighted to match the pileup profile observed in the collected data. 


\section{Event reconstruction and selection}

Events are reconstructed using the CMS particle-flow (PF) algorithm [78], which seeks to reconstruct and identify the individual particles in the event via an optimal combination of all information in the CMS detector. The reconstructed particles are identified as charged or neutral hadrons, electrons, muons, or photons, and constitute a list of PF candidate physics objects. At least one reconstructed vertex is required. In the case of multiple collision vertices from pileup interactions, the candidate vertex with the largest value of summed physics-object $p_{\mathrm{T}}^{2}$ is taken to be the primary pp interaction vertex. The physics objects are the jets, clustered using the jet finding algorithm $[79,80]$ with the tracks assigned to candidate vertices as inputs, and the associated missing transverse momentum, taken as the negative vector sum of the $p_{\mathrm{T}}$ of those jets. Events affected by reconstruction failures, detector malfunctions, or noncollision backgrounds, are identified and rejected by dedicated filters [81].

Electrons are reconstructed by combining information from the tracker and energy deposits in the ECAL [82]. Muons are reconstructed by combining information from the tracker and the muon system [47]. Only tracks originating from the PV can be associated with the electrons or muons, and quality criteria [47, 82] are further imposed that obtain more pure identification without substantial loss of efficiency. To suppress leptons stemming from $\mathrm{b}$ and $\mathrm{c}$ decays, while retaining leptons from $\mathrm{V}$ decays, isolation is required from jet activity within a cone of radius $\Delta R=\sqrt{(\Delta \eta)^{2}+(\Delta \phi)^{2}}=0.3$. The isolation is defined as the scalar $p_{\mathrm{T}}$ sum of the PF candidates within the cone divided by the lepton $p_{\mathrm{T}}$. The upper threshold applied on the relative isolation is 0.06 for electrons and muons in the $\mathrm{W}(\ell v) \mathrm{H}(\mathrm{c} \overline{\mathrm{c}})$ channel and 0.15 and 0.25 for electrons and muons respectively in the $\mathrm{Z}(\ell \ell) \mathrm{H}(\mathrm{c} \overline{\mathrm{c}})$ channel. Charged $\mathrm{PF}$ candidates not originating from the $\mathrm{PV}$, as well as $\mathrm{PF}$ candidates identified as electrons or muons, are not considered in the sum [83]. The isolation of electrons and muons is also corrected for the estimated energy that is contributed to the isolation region by neutral particles originating from pileup. In the case of electrons, the latter is estimated by an effective jet area from the measured neutral energy density [82], while for muons, the $\Delta \beta$-correction method [47] is applied.

Jets are reconstructed by clustering the PF candidates with the anti- $k_{\mathrm{T}}$ algorithm $[79$, 80] using a distance parameter $R$. The jet momentum is determined as the vectorial sum of all PF candidate momenta in the jet, and is found in simulation to be within 5 to $10 \%$ of the true momentum over the full detector acceptance and range of $p_{\mathrm{T}}$ considered in this analysis. The raw jet energies are then corrected to establish a uniform response of the calorimeter in $\eta$ and a calibrated absolute response in $p_{\mathrm{T}}$. Additional corrections to account for any residual differences between the jet energy scale in data and simulation are extracted and applied based on comparison of data and simulated samples in relevant control regions [84]. The jet energy resolution typically amounts to $15-20 \%$ at $30 \mathrm{GeV}$, about $10 \%$ at $100 \mathrm{GeV}$, and $5 \%$ at $1 \mathrm{TeV}$ [84]. Corrections extracted from data control regions are applied to account for the difference between the jet energy resolution in data and simulation. Additional selection criteria are applied to each jet to remove those that are potentially dominated by instrumental or reconstruction failures [85]. 
Two collections of jets reconstructed with the anti- $k_{\mathrm{T}}$ algorithm are used in the search. The first consists of jets clustered with $R=0.4$, and will be referred to as "small- $R$ jets". The charged hadron subtraction algorithm $[86]$ is used to eliminate PF candidates from the jet constituents associated with vertices from pileup interactions. The neutral component of the energy arising from pileup interactions is estimated with the effective area method [85]. The small- $R$ jets are required to have $p_{\mathrm{T}}>20 \mathrm{GeV}$ and to be within the tracker acceptance, $|\eta|<2.4$. Any small- $R$ jets that overlap with preselected electrons and muons, as defined by $\Delta R(\mathrm{j}, \ell)<0.4$, are discarded.

The second jet collection is based on jets reconstructed using $R=1.5$. This collection will be referred to as "large- $R$ jets" in what follows. In this case, the PUPPI algorithm [87] is used to correct the jet energy for contributions coming from pileup. Additional information on jet substructure is obtained by reclustering the constituents of these jets via the Cambridge-Aachen algorithm [88]. The "modified mass drop tagger" algorithm [89, 90], also known as the "soft-drop" (SD) algorithm, with angular exponent $\beta=0$, soft cutoff threshold $z_{\text {cut }}=0.1$, and characteristic radius $R_{0}=1.5$ [91], is applied to remove soft, wide-angle radiation from the jet. In the default configuration, the SD algorithm identifies two hard subjets within the large- $R$ jet by reversing the Cambridge-Aachen clustering history. The kinematic variables of the two subjets are used to calculate the 4-momentum of the large- $R$ jet. The large- $R$ jets are required to have $|\eta|<2.4$ and a soft drop mass of $50<m_{\mathrm{SD}}<200 \mathrm{GeV}$. Large- $R$ jets that overlap with preselected electrons and muons, as defined by $\Delta R(\mathrm{j}, \ell)<1.5$, are discarded.

The missing transverse momentum vector $\vec{p}_{\mathrm{T}}^{\text {miss }}$ is computed as the negative vector $p_{\mathrm{T}}$ sum of all the PF candidates in an event, and its magnitude is denoted $p_{\mathrm{T}}^{\text {miss }}[81]$. The magnitude and direction of $\vec{p}_{\mathrm{T}}^{\text {miss }}$ are modified to account for corrections to the energy scale of the reconstructed jets in the event.

One of the most challenging tasks of this analysis is the discrimination of jets that are the result of the hadronisation of $\mathrm{c}$ quarks from all other jet flavours. Tagging $\mathrm{c}$ jets is more difficult than tagging $\mathrm{b}$ jets because they are less distinct from light-flavour quark or gluon jets (udsg) in regard to mass, decay length of charmed hadrons produced in the hadronisation process, and multiplicity of tracks inside the jet. The resolved- and mergedjet topology analyses use different strategies for tagging c jets. More details on c tagging are presented below in sections 5 and 6 .

\subsection{Baseline selection}

The search uses the leptonic decays of the vector bosons to define three mutually exclusive channels based on the charged-lepton multiplicity in the final state, namely: "OL" channel as referring to the $\mathrm{Z}(v v) \mathrm{H}(\mathrm{c} \overline{\mathrm{c}})$ signal process, " $1 \mathrm{~L}$ " channel as referring to the $\mathrm{W}(\ell v) \mathrm{H}(\mathrm{c} \overline{\mathrm{c}})$ signal process, and " $2 \mathrm{~L}$ " channel as referring to the $\mathrm{Z}(\ell \ell) \mathrm{H}(\mathrm{c} \overline{\mathrm{c}})$ signal process. The $1 \mathrm{~L}$ and $2 \mathrm{~L}$ channels are further subdivided based on lepton flavour. Only electrons and muons are considered in this search.

Events in the $0 \mathrm{~L}$ channel are collected with a trigger requiring the presence of $p_{\mathrm{T}}^{\text {miss }}$ above $170 \mathrm{GeV}$ or $110 \mathrm{GeV}$ and an additional threshold on the missing hadronic transverse energy of $110 \mathrm{GeV}$. Events in the $1 \mathrm{~L}$ channel are obtained with a trigger requiring the 
presence of an isolated electron or muon with $p_{\mathrm{T}}$ above 27 and $24 \mathrm{GeV}$, respectively. Events in the $2 \mathrm{~L}$ channel of the resolved-jet topology analysis are selected by triggers that require the presence of a pair of leptons with $p_{\mathrm{T}}$ larger than 23 and $12 \mathrm{GeV}$ for electrons, and 17 and $8 \mathrm{GeV}$ for muons. The same dielectron trigger has been used in the $2 \mathrm{~L} \mathrm{Z}$ (ee) channel of the merged-jet topology analysis, while events in the $\mathrm{Z}(\mu \mu)$ channel are selected by the above single-muon trigger, which provides high efficiency for muons produced in the decays of high- $p_{\mathrm{T}}$ bosons.

The collected events are required to pass additional offline criteria. In the 0L channel corresponding to $\mathrm{Z}$ boson decays to neutrinos, $p_{\mathrm{T}}^{\text {miss }}>170 \mathrm{GeV}$ is required and events with identified isolated leptons are rejected. The $\vec{p}_{\mathrm{T}}^{\text {miss }}$ is taken to correspond to $\vec{p}_{\mathrm{T}}(\mathrm{V})$ in this case. Events with a single electron (muon) with $p_{\mathrm{T}}>30(25) \mathrm{GeV}$ pass the $1 \mathrm{~L}$ selection. The leptonically decaying $\mathrm{W}$ boson is approximately reconstructed as the vectorial sum of the lepton momentum and $\vec{p}_{\mathrm{T}}^{\text {miss }}$. The event topology is required to be compatible with the leptonic decay of a Lorentz-boosted $\mathrm{W}$ boson by requiring $\Delta \phi\left(p_{\mathrm{T}}^{\mathrm{miss}}, \ell\right)<2.0(1.5)$ in the resolved-jet (merged-jet) topology analysis. Finally, for the $2 \mathrm{~L}$ selection, the two highest $p_{\mathrm{T}}$ leptons are required to be of the same flavour, opposite electric charge, and to have a $p_{\mathrm{T}}$ above $20 \mathrm{GeV}$. The $\mathrm{Z}$ boson candidates are then reconstructed as the sum of the four-momenta of these two leptons, and the invariant mass of the candidates is required to be compatible with the $\mathrm{Z}$ boson mass $\left(75<m_{\ell \ell}<105 \mathrm{GeV}\right)$.

A typical $\mathrm{VH}(\mathrm{H} \rightarrow \mathrm{c} \overline{\mathrm{c}})$ event has the signature of a vector boson recoiling against a $\mathrm{H}$ boson with little additional activity. The event selection is designed to retain such events while suppressing background processes as much as possible. In addition to the requirement of a high $-p_{\mathrm{T}}$ vector boson, the QCD multijet background is reduced to negligible levels by demanding the $\vec{p}_{\mathrm{T}}^{\text {miss }}$ to not be aligned with any jet in the event and requiring the azimuthal angular separation $\Delta \phi\left(\vec{p}_{\mathrm{T}}^{\text {miss }}\right.$ trk,$\left.\vec{p}_{\mathrm{T}}^{\text {miss }}\right)<0.5$ for which $\vec{p}_{\mathrm{T}}^{\text {miss }}$ trk is calculated solely from charged particles. This latter selection reduces the contribution of QCD multijet events that arise from the presence of "fake" $\vec{p}_{\mathrm{T}}^{\text {miss }}$ coming from jet energy mismeasurement in the calorimeters. A significant fraction of the t $\bar{t}$ background is suppressed by rejecting events with $\mathrm{N}_{\mathrm{small}-R}^{\mathrm{aj}}>1$ in the $0 \mathrm{~L}$ and $1 \mathrm{~L}$ channels, and $\mathrm{N}_{\mathrm{small}-R}^{\mathrm{aj}}>2$ in the $2 \mathrm{~L}$ channel of the merged-jet topology analysis, where $\mathrm{N}_{\text {small- } R}^{\text {aj }}$ represents the additional small- $R$ jet multiplicity. This requirement is not needed in the $2 \mathrm{~L}$ channel of the resolved-jet topology analysis where the top quark background is negligible.

The dominant background that remains after the application of the event selection described above is $\mathrm{V}+$ jets. The contribution from this background is suppressed by requiring the dijet invariant mass $m_{\mathrm{jj}}$ (calculated using two small- $R$ jets) in the resolved-jet topology analysis and $m_{\mathrm{SD}}$ in the merged-jet topology analysis to satisfy $50<m_{\mathrm{jj}}\left(m_{\mathrm{SD}}\right)<200 \mathrm{GeV}$. Contributions from $t \overline{\mathrm{t}}$ and single top processes remain significant in the $0 \mathrm{~L}$ and $1 \mathrm{~L}$ channels because of the presence of at least one $\mathrm{W}$ boson and because $\mathrm{b}$ quarks are often misidentified as c quarks by the c tagging algorithms. Contributions from diboson processes are typically small as a result of their small production cross sections. The background originating from $\mathrm{H}$ bosons decaying into $\mathrm{b}$ quarks presents kinematic properties similar to those of the signal, with the exception of a higher average energy of neutrinos in b jets than in $c$ jets. This background is reduced by exploiting dedicated jet flavour taggers, as described in sections 5 and 6. 
The details of the resolved-jet topology and merged-jet topology analyses are described in sections 5 and 6 , respectively. Section 7 is dedicated to the treatment of the systematic uncertainties and section 8 presents the results of the two analyses and of their combination. Section 8 presents also the strategy that is used to make the two analyses mutually exclusive in order to facilitate their combination for the final results.

\section{$5 \quad$ Resolved-jet topology analysis}

Approximately $95 \%$ of the VH events produced at $\sqrt{s}=13 \mathrm{TeV}$ have a vector boson with $p_{\mathrm{T}}$ lower than $200 \mathrm{GeV}$, corresponding to the phase space region where the $\mathrm{H}$ boson decay products generally give rise to two distinctly reconstructed small- $R$ jets in the CMS detector. The resolved-jet analysis aims to exploit a large fraction of this phase space which, however, contains a sizeable background contamination. The requirement of a moderate boost of the vector boson is then found to be crucial to the reduction of $\mathrm{V}+$ jets and $\mathrm{t} \overline{\mathrm{t}}$ backgrounds. Dedicated charm taggers based on ML are used to order the jets in the event by their likelihood to be $\mathrm{c}$ jets that are considered for use in reconstructing the $\mathrm{H}$ boson candidate.

Backgrounds arise from the production of $\mathrm{W}$ and $\mathrm{Z}$ bosons in association with one or more jets, single and pair-produced top quarks, and diboson events. A small residual QCD background is present in the $0 \mathrm{~L}$ and $1 \mathrm{~L}$ channels. High-purity control regions for the $\mathrm{V}+\mathrm{udsg}$ and $\mathrm{t} \overline{\mathrm{t}}$ backgrounds are identified in data and used to estimate expected yields of these backgrounds in the signal region. Samples of events in regions that are disjoint from the signal region in c tagging probability and dijet mass but which are enhanced in $\mathrm{V}+\mathrm{b} \overline{\mathrm{b}}$ and $\mathrm{V}+\mathrm{c} \overline{\mathrm{c}}$ production are used to provide data-driven constraints on the $\mathrm{V}+\mathrm{b} \overline{\mathrm{b}}$ and $\mathrm{V}+\mathrm{c} \overline{\mathrm{c}}$ backgrounds, respectively. Finally, a binned maximum likelihood fit is carried out simultaneously in the signal region and in the control regions for all channels.

\subsection{Higgs boson reconstruction}

The $\mathrm{H}$ candidate is reconstructed as two distinct small- $R$ jets. The identification of $\mathrm{c}$ jets among those arising from other flavours of quarks or gluons is achieved with the Deep Combined Secondary Vertex (DeepCSV) algorithm [44]. This algorithm encodes a multiclassifier based on advanced ML techniques and provides three output weights $p(\mathrm{~b}), p(\mathrm{c})$, and $p(\mathrm{udsg})$ which can be interpreted as the probabilities for a given jet to have originated from a bottom quark, a charm quark, or a gluon or light-flavour quark, respectively. By combining the various DeepCSV outputs, it is possible to define two discriminators for c tagging. The inputs to the DeepCSV algorithm are variables constructed from observables associated with the reconstructed primary and secondary vertices, tracks, and jets. The discrimination between c jets and light-flavour quark or gluon jets is achieved via the probability ratio defined as $C v s L=p(\mathrm{c}) /[p(\mathrm{c})+p(\mathrm{udsg})]$. In the same way, discrimination between $\mathrm{c}$ jets and $\mathrm{b}$ jets makes use of the probability ratio defined as $C v s B=p(\mathrm{c}) /[p(\mathrm{c})+p(\mathrm{~b})]$. The two discriminator ratio values for each jet define a two-dimensional distribution. The resulting $\mathrm{c}$ tagging efficiency as a function of the $\mathrm{b}$ jet and light-flavour quark or gluon jet efficiencies is shown in figure 1 . To account for residual $\mathrm{O}(10 \%)$ differences in the distributions of $C v s L$ and $C v s B$ found in the comparison of data and simulation, reshaping scale 


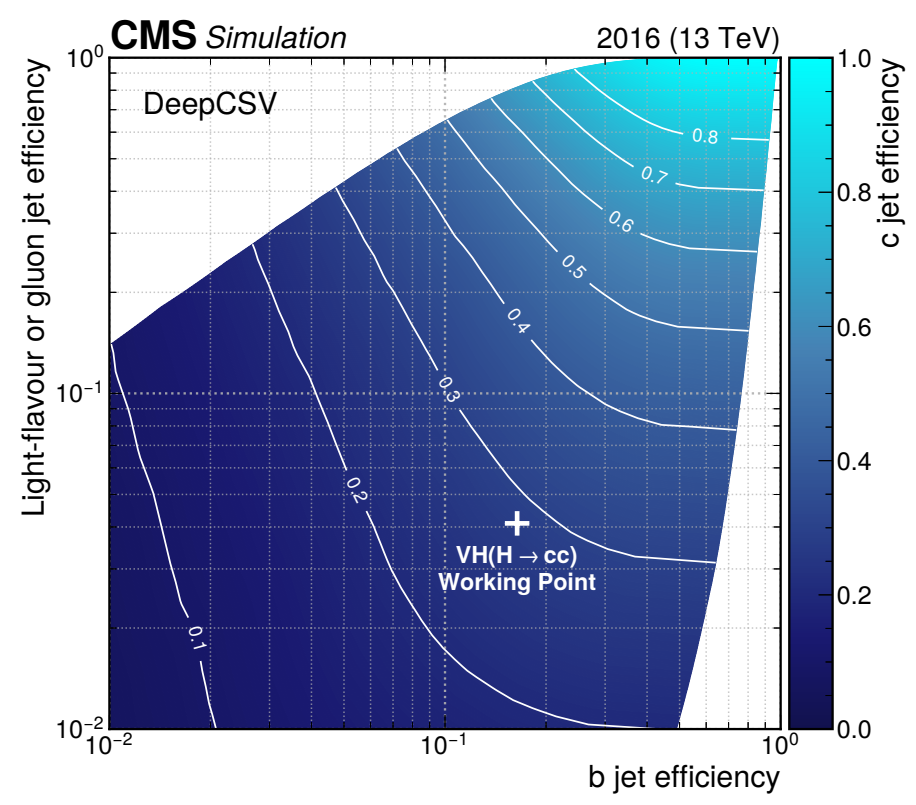

Figure 1. Efficiency to tag a $\mathrm{c}$ jet as a function of the b jet and light-flavour quark or gluon jet mistag rates. The working point adopted in the resolved-jet topology analysis to select the leading CvsL jets is shown with a white cross. The white lines correspond to $\mathrm{c}$ jet iso-efficiency curves. The plot makes use of jets with $p_{\mathrm{T}}>20 \mathrm{GeV}$ that have been clustered with AK4 algorithm in a simulated $t \bar{t}+$ jets sample before application of data-to-simulation reshaping scale factors.

factors have been extracted using an iterative fit to distributions in control regions enriched in Drell-Yan+jets, semileptonic $t \overline{\mathrm{t}}+$ jets, and $\mathrm{W}+\mathrm{c}$ events that provide data samples with large fractions of light-flavour quark or gluon jets, b jets, and c jets, respectively. The corresponding uncertainties, evaluated on a per jet basis as a function of the jet flavour, range from $2 \%$ for bottom, gluon, and light-flavoured quark jets to $5 \%$ for c jets.

The probability ratios $C v s L$ and $C v s B$ are used to discriminate candidates that are consistent with the $\mathrm{c}$ jet hypothesis from jets originating from light-flavour quarks or gluons, and b quarks, respectively. The two jets with the highest score of CvsL in the event are chosen to build the $\mathrm{H}$ candidate four-vector. Events are required to have the leading jet in $C v s L$ score passing the c tagger working point requirements $(C v s L>0.4, C v s B>$ 0.2 ). This working point has been chosen such that the efficiency to identify a $\mathrm{c}$ jet is approximately $28 \%$, while the misidentification rate is $4 \%$ for light-flavour quark or gluon jets and $15 \%$ for b jets. The misidentification rate of $\tau$ leptons as $\mathrm{c}$ jets is larger than the misidentification rate of $\mathrm{b}$ jets as $\mathrm{c}$ jets. However, the kinematic properties of the $\tau$ decays are exploited by the BDTs, as described in section 5.2, to discriminate signal c jet pairs from misidentified jet pairs coming from $\tau$ leptons. The contribution of the $\mathrm{VH}(\mathrm{H} \rightarrow \tau \tau)$ process in the high signal purity bins of the BDT distributions has a negligible impact on the final results. To account for jets originating from final-state radiation (FSR), additional jets with $p_{\mathrm{T}}>30 \mathrm{GeV}$ and $|\eta|<3.0$ are included in the calculation of the components of 
the $\mathrm{H}$ candidate four-vector if they lie in a cone of $\Delta R<0.8$ centred on the direction of one of the two leading jets.

\subsection{Signal extraction}

In addition to the selections reported in sections 4.1 and 5.1, in the $1 \mathrm{~L}$ and $0 \mathrm{~L}$ channels of the resolved-jet topology analysis, where larger backgrounds are expected, the $\mathrm{V}$ candidates are required to have $p_{\mathrm{T}}$ of at least 100 and $170 \mathrm{GeV}$ respectively, while, for the same channels, the $\mathrm{H}$ candidates are required to have $p_{\mathrm{T}}$ of at least 100 and $120 \mathrm{GeV}$. In the $2 \mathrm{~L}$ channel, where the background from $t \overline{\mathrm{t}}$ production is much smaller and the effective signal cross section is also lower, two regions are considered: a low- $p_{\mathrm{T}}(\mathrm{V})$ region defined by $50<p_{\mathrm{T}}(\mathrm{V})<150 \mathrm{GeV}$ and a high- $p_{\mathrm{T}}(\mathrm{V})$ region with $p_{\mathrm{T}}(\mathrm{V})>150 \mathrm{GeV}$ (no upper cut is applied on $\left.p_{\mathrm{T}}(\mathrm{V})\right)$. In $\mathrm{VH}(\mathrm{H} \rightarrow \mathrm{c} \overline{\mathrm{c}})$ signal events, the vector boson is typically produced in the azimuthal direction opposite to that of the $\mathrm{H}$ boson. Therefore, an additional requirement on the difference in azimuthal angle between the reconstructed $\mathrm{V}$ and $\mathrm{H}$ candidate, $\Delta \phi(\mathrm{V}, \mathrm{H})>2.5(>2.0$ in the $0 \mathrm{~L}$ channel), is applied.

In the signal regions defined by the application of the selection criteria mentioned above, a boosted decision tree (BDT) with gradient boost [92] has been trained to enhance the signal separation from background. The same simulated samples, each normalised to the cross section of the relevant physics process, have been split into two independent subsets used for training and testing the BDTs. All the data-to-simulation scale factors relative to trigger efficiency, lepton identification and isolaton efficiency, and c-tagging have been applied to the simulated samples. Separate BDTs have been trained for $0 \mathrm{~L}, 1 \mathrm{~L}$, and $2 \mathrm{~L}$ $\left(\right.$ low- $p_{\mathrm{T}}(\mathrm{V})$ and high- $\left.p_{\mathrm{T}}(\mathrm{V})\right)$ channels. The muon and electron samples were combined to train the BDTs to benefit from a higher number of simulated events. The distributions of all variables used to construct the BDT discriminator and hence the BDT distribution itself are taken from simulation after the application of the corrections detailed in section 3. Table 1 lists the input variables considered in each channel. As expected, the most discriminating variables are found to be the $\mathrm{H}$ candidate invariant mass and the $C v s L_{\max }$.

The remaining background contribution is estimated from a combination of simulated events and data. While the normalisations of QCD, single-top, diboson, and $\mathrm{VH}(\mathrm{H} \rightarrow$ $\mathrm{b} \overline{\mathrm{b}})$ processes are estimated via simulation, the normalisations of the $\mathrm{V}+$ jets and $t \bar{t}+\mathrm{jets}$ backgrounds are determined from fits to data in dedicated control regions in order to avoid potential mismodelling of the flavour composition of these samples. Four control regions per channel are designed to constrain the most important background processes: a region dominated by $t \bar{t}+$ jets events $(\mathrm{TT})$, a region targeting $\mathrm{V}+$ jets with at least one jet originating from light-flavour quarks or gluons (LF), a region enriched in $\mathrm{V}+$ jets events with one $b$ jet and one $b$ or $c$ jet $(\mathrm{HF})$, and a region enriched with $\mathrm{V}+\mathrm{c} \overline{\mathrm{c}}$ events $(\mathrm{CC})$. The definitions of the different control regions are based mainly on the inversion of the criteria on the charm tagger discriminators values of the CvsL-leading jet applied to define the signal regions. To define the LF control region the selection $(C v s L<0.4, C v s B>$ 0.2 ) is used while both the HF and TT control regions are defined applying the selection $(C v s L>0.4, C v s B<0.2)$. In order to differentiate the TT from HF control regions, further requirements are applied such as a veto on the reconstructed $\mathrm{Z}$ boson mass in the 


\begin{tabular}{|c|c|c|c|c|}
\hline Variable & Description & $0 \mathrm{~L}$ & $1 \mathrm{~L}$ & $2 \mathrm{~L}$ \\
\hline$m(\mathrm{H})$ & $\mathrm{H}$ mass & $\checkmark$ & $\checkmark$ & $\checkmark$ \\
\hline$p_{\mathrm{T}}(\mathrm{H})$ & $\mathrm{H}$ transverse momentum & $\checkmark$ & $\checkmark$ & $\checkmark$ \\
\hline$p_{\mathrm{T}}(\mathrm{V})$ & vector boson transverse momentum & $\checkmark$ & $\checkmark$ & $\checkmark$ \\
\hline$m(\mathrm{~V})$ & vector boson mass & - & - & $\checkmark$ \\
\hline$m_{\mathrm{T}}(\mathrm{V})$ & vector boson transverse mass & - & $\checkmark$ & - \\
\hline$p_{\mathrm{T}}^{\mathrm{miss}}$ & missing transverse momentum & $\checkmark$ & $\checkmark$ & - \\
\hline$p_{\mathrm{T}}(\mathrm{V}) / p_{\mathrm{T}}(\mathrm{H})$ & ratio between vector boson and $\mathrm{H}$ transverse momenta & $\checkmark$ & $\checkmark$ & $\checkmark$ \\
\hline$C v s L_{\max }$ & $C v s L$ value of the leading $C v s L$ jet & $\checkmark$ & $\checkmark$ & $\checkmark$ \\
\hline$C v s B_{\max }$ & $C v s B$ value of the leading $C v s L$ jet & $\checkmark$ & $\checkmark$ & $\checkmark$ \\
\hline$C v s L_{\min }$ & $C v s L$ value of the subleading $C v s L$ jet & $\checkmark$ & $\checkmark$ & $\checkmark$ \\
\hline$C v s B_{\min }$ & $C v s B$ value of the subleading $C v s L$ jet & $\checkmark$ & $\checkmark$ & $\checkmark$ \\
\hline$p_{\text {Tmax }}$ & $p_{\mathrm{T}}$ of the leading $C v s L$ jet & $\checkmark$ & $\checkmark$ & $\checkmark$ \\
\hline$p_{\text {Tmin }}$ & $p_{\mathrm{T}}$ of the subleading $C v s L$ jet & $\checkmark$ & $\checkmark$ & $\checkmark$ \\
\hline$\Delta \phi(\mathrm{V}, \mathrm{H})$ & azimuthal angle between vector boson and $\mathrm{H}$ & $\checkmark$ & $\checkmark$ & $\checkmark$ \\
\hline$\Delta R\left(\mathrm{j}_{1}, \mathrm{j}_{2}\right)$ & $\Delta R$ between leading and subleading $C v s L$ jets & - & $\checkmark$ & $\checkmark$ \\
\hline$\Delta \phi\left(\mathrm{j}_{1}, \mathrm{j}_{2}\right)$ & azimuthal angle between leading and subleading $C v s L$ jets & $\checkmark$ & $\checkmark$ & - \\
\hline$\Delta \eta\left(\mathrm{j}_{1}, \mathrm{j}_{2}\right)$ & difference in pseudorapidity between leading and subleading $C v s L$ jets & $\checkmark$ & $\checkmark$ & $\checkmark$ \\
\hline$\Delta \phi\left(\ell_{1}, \ell_{2}\right)$ & azimuthal angle between leading and subleading $p_{\mathrm{T}}$ leptons & - & - & $\checkmark$ \\
\hline$\Delta \eta\left(\ell_{1}, \ell_{2}\right)$ & difference in pseudorapidity between leading and subleading $p_{\mathrm{T}}$ leptons & - & - & $\checkmark$ \\
\hline$\Delta \phi\left(\ell_{1}, \mathrm{j}_{1}\right)$ & azimuthal angle between leading $p_{\mathrm{T}}$ lepton and leading $C v s L$ jet & - & $\checkmark$ & - \\
\hline$\Delta \phi\left(\ell_{2}, \mathrm{j}_{1}\right)$ & azimuthal angle between subleading $p_{\mathrm{T}}$ lepton and leading $C v s L$ jet & - & - & $\checkmark$ \\
\hline$\Delta \phi\left(\ell_{2}, \mathrm{j}_{2}\right)$ & azimuthal angle between subleading $p_{\mathrm{T}}$ lepton and subleading $C v s L$ jet & - & - & $\checkmark$ \\
\hline$\Delta \phi\left(\ell_{1}, p_{\mathrm{T}}^{\mathrm{miss}}\right)$ & azimuthal angle between leading $p_{\mathrm{T}}$ lepton and missing transverse momentum & - & $\checkmark$ & \\
\hline $\mathrm{N}_{\mathrm{small}-R}^{\mathrm{aj}}$ & number of small- $R$ jets minus the number of FSR jets & $\checkmark$ & $\checkmark$ & \\
\hline$N_{5}^{\text {soft }}$ & multiplicity of soft track-based jets with $p_{\mathrm{T}}>5 \mathrm{GeV}$ & $\checkmark$ & $\checkmark$ & \\
\hline
\end{tabular}

Table 1. Variables employed in the training of the BDT used for each channel of the resolved-jet topology analysis. The $2 \mathrm{~L}$ case has separate training for the low- and high- $p_{\mathrm{T}}(\mathrm{V})$ channels, but exploits the same input variables.

$2 \mathrm{~L}$ channel, $m_{\ell \ell} \notin[75,120] \mathrm{GeV}$, and the requirement $\mathrm{N}_{\text {small- } R}^{\mathrm{aj}} \geq 2$. The $\mathrm{CC}$ control region is defined identically to the signal region, except for inverting the requirement on the $\mathrm{H}$ mass. The simulated $\mathrm{V}+$ jets backgrounds are similarly split into four classes depending on the flavour(s) of the additional jet(s) present in the processes: $\mathrm{V}+2$ light-flavour quark or gluon jets, $\mathrm{V}+\mathrm{udsg}$ and $1 \mathrm{~b}$ or $1 \mathrm{c}, \mathrm{V}+\mathrm{bb}$ or $\mathrm{bc}$, and $\mathrm{V}+\mathrm{cc}$ jets.

Separate normalisation scale factors are used to constrain $\mathrm{Z}+$ jets processes in the $\mathrm{OL}$ and $2 \mathrm{~L}$ channels, while the normalisation scale factors related to $\mathrm{W}+$ jets processes are shared between the $0 \mathrm{~L}$ and $1 \mathrm{~L}$ analysis channels. To constrain the $t \overline{\mathrm{t}}+$ jets process, on the other hand, each channel relies on its own independent normalisation scale factors. The normalisation scale factors are measured together with the signal strength modifier through a simultaneous fit to data in all control and signal regions for all of the analysis channels. The simulated diboson background is split according to the presence or absence 
of a $\mathrm{Z}$ boson decaying to a pair of charm quarks, labelling them as $\mathrm{VZ}(\mathrm{Z} \rightarrow \mathrm{c} \overline{\mathrm{c}})$ if such a $\mathrm{Z}$ boson decay is present and $\mathrm{VV}+$ other otherwise. Whereas in the signal regions the BDT discriminator is used for the final signal extraction, in the control regions the shape of the CvsB distribution is used in the TT, HF, and $\mathrm{CC}$ regions, while that of $C v s L$ is used in the $\mathrm{LF}$ region. The reason of this choice lies in the fact that $C v s B$ provides the best discriminant between $\mathrm{b}$ and $\mathrm{c}$ jets and thus it is used in the control regions where there is an enhanced presence of $\mathrm{b}$ jets, while $C v s L$ is more efficient in separating light-flavour quark or gluon jets from c jets and hence it is preferred in the LF control region.

Figure 2 shows the distributions of the $C v s B$ discriminant for the subleading $C v s L$ jet for the $\mathrm{HF}$ and $\mathrm{CC}$ control regions in the $2 \mathrm{~L}(\mathrm{Z}(\mu \mu))$ low- $p_{\mathrm{T}}(\mathrm{V}), 2 \mathrm{~L}(\mathrm{Z}(\mathrm{ee}))$ high- $p_{\mathrm{T}}(\mathrm{V})$, $1 \mathrm{~L}(\mathrm{~W}(\mu \nu))$, and $0 \mathrm{~L}$ channels. The post-fit distributions (for fit details, see section 8 ) in figure 2 show good agreement between the data and the simulation in these two most significant control regions. Moreover, the employment of the full distribution of the $C v s B$ score provides a good separation between the $\mathrm{V}+\mathrm{bb}$ and $\mathrm{V}+\mathrm{cc}$ processes that makes it possible to constrain these two backgrounds. The corresponding distributions for the other channels are not shown but are similar in their behaviour.

\section{Merged-jet topology analysis}

For the case of a Lorentz-boosted $\mathrm{H}$ boson as flagged by a $\mathrm{V}$ boson with $p_{\mathrm{T}}(\mathrm{V}) \gtrsim 200 \mathrm{GeV}$, a merged-jet topology is considered. The dominant backgrounds after the baseline selection presented in section 4.1 come from $\mathrm{V}+$ jets and $\mathrm{t} \overline{\mathrm{t}}$ processes. The $\mathrm{V}$ bosons in the signal process have on average larger $p_{\mathrm{T}}$ than those from the $\mathrm{V}+$ jets background. The analysis focuses on the reconstruction of moderately to highly Lorentz-boosted $\mathrm{H}$ bosons where the decay products are contained in a single large- $R$ jet. Dedicated object reconstruction tools based on large- $R$ jets and advanced ML techniques were developed to identify and reconstruct Lorentz-boosted $\mathrm{H}$ bosons decaying to charm quarks.

\subsection{Higgs boson reconstruction}

The cornerstone of the merged-jet topology analysis is the reconstruction of the $\mathrm{H} \rightarrow \mathrm{c} \overline{\mathrm{c}}$ candidate in a single, large- $R$ jet, which has the potential to provide a better signal purity because the signal has a tendency to be more boosted than the dominant $V+$ jets and $t \overline{\mathrm{t}}$ backgrounds, as noted above. In view of this, the high- $p_{\mathrm{T}}$ regime with $p_{\mathrm{T}}(\mathrm{V}) \gtrsim 200 \mathrm{GeV}$, though representing no more than approximately $5 \%$ of the total phase space, can provide a significant contribution to the search. Moreover, the merged-jet approach has important advantages over the resolved-jet approach at high $p_{\mathrm{T}}$. The possibility for both c quarks to reside in a single large- $R$ jet enhances the signal acceptance, improves the identification of the correct pair of jets to use in reconstructing the $\mathrm{H}$ boson, and similarly facilitates the task of taking into account any FSR that may have been emitted by the quarks. A more detailed discussion of the potential advantages of this approach can be found in refs. [90, 93]. Given the small fraction of signal events that survive a selection with $p_{\mathrm{T}}(\mathrm{V}) \gtrsim 200 \mathrm{GeV}$, it is critical to carefully choose the $R$ parameter of the jet clustering algorithm. In general, the angular separation between the decay products of a Lorentz-boosted particle such as the 

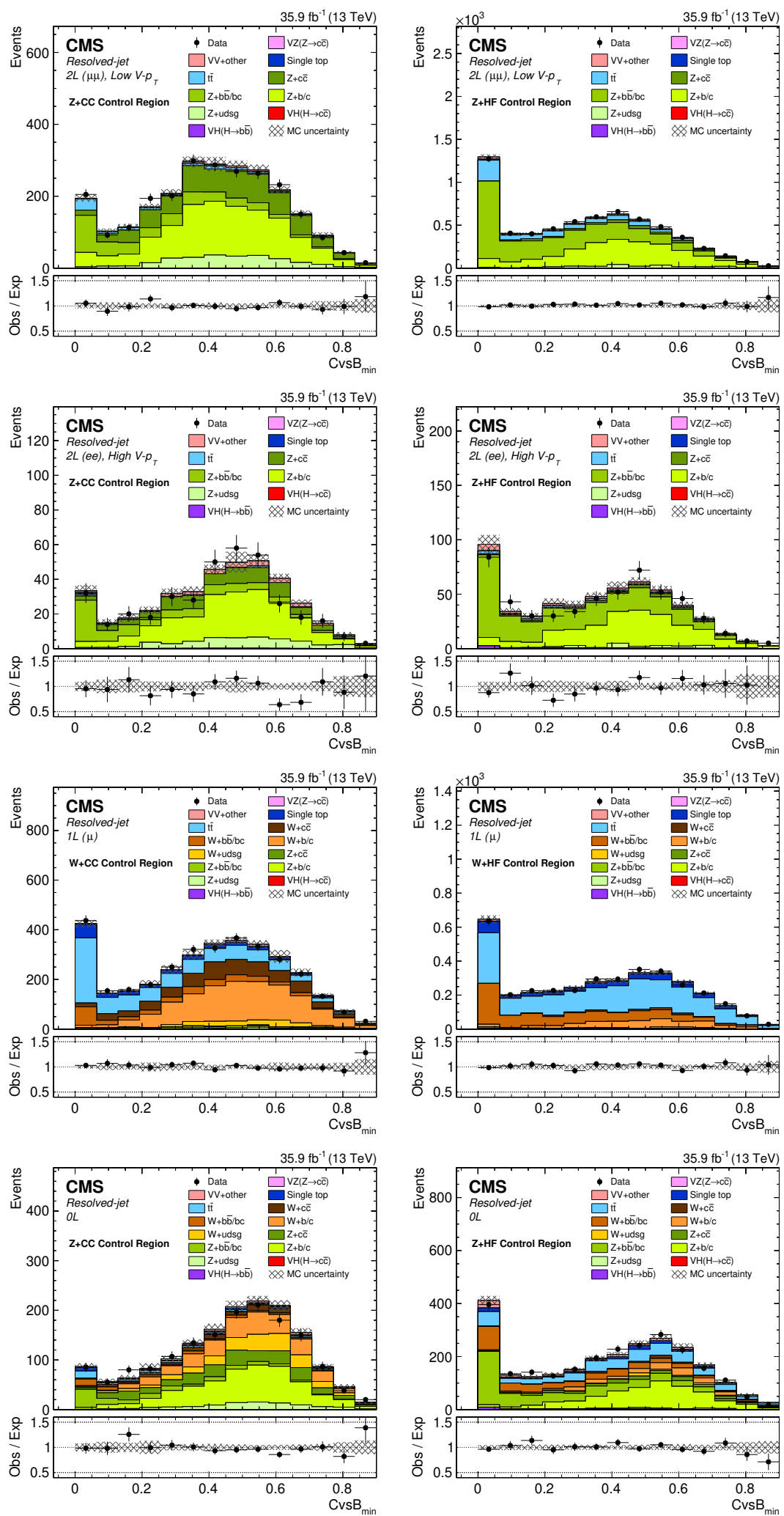

Figure 2. Post-fit $C v s B_{\min }$ distributions in the $\mathrm{CC}$ (left panel) and HF (right panel) control regions for the $2 \mathrm{~L}(\mathrm{Z}(\mu \mu))$ low- $p_{\mathrm{T}}(\mathrm{V}), 2 \mathrm{~L}(\mathrm{Z}(\mathrm{ee}))$ high- $p_{\mathrm{T}}(\mathrm{V}), 1 \mathrm{~L}(\mathrm{~W}(\mu \nu))$, and $0 \mathrm{~L}$ channels. 
$\mathrm{H}$ boson is approximately given by $\Delta R \sim 2 m_{\mathrm{H}} / p_{\mathrm{T}}(\mathrm{H})$. For a $p_{\mathrm{T}}(\mathrm{H}) \sim p_{\mathrm{T}}(\mathrm{V})$ of $200 \mathrm{GeV}$, this gives $\Delta R \approx 1.25$. For good signal purity and acceptance, we have thus chosen to use large- $R$ jets clustered by the anti- $k_{\mathrm{T}}$ algorithm with a distance parameter of $R=1.5$.

As for the resolved-jet topology analysis, one of the biggest challenges is the efficient reconstruction of the pair of c quarks from the $\mathrm{H}$ boson decay, while also achieving significant rejection of both light-flavour quarks and gluons, as well as b quarks that contribute backgrounds to this search. To this end, a novel algorithm, DeepAK15 [45], has been used for the identification of jet substructure to tag $\mathrm{W}, \mathrm{Z}$, and $\mathrm{H}$ bosons, as well as top quarks. In addition, DeepAK15 is designed to discriminate between decay modes with different flavour content (e.g. $\mathrm{H} \rightarrow \mathrm{b} \overline{\mathrm{b}}, \mathrm{H} \rightarrow \mathrm{c} \overline{\mathrm{c}}, \mathrm{H} \rightarrow \mathrm{q} \overline{\mathrm{q}} \mathrm{q} \overline{\mathrm{q}}$ ). The algorithm deploys ML methods on the PF candidates and secondary vertices, which are used as inputs. DeepAK15 is designed to exploit information related to jet substructure, flavour, and pileup simultaneously, yielding substantial gains with respect to other approaches [45]. With the use of the adversarial training procedure [94], the algorithm is largely decorrelated from the jet mass, while preserving most of the method's discriminating power.

The performance in terms of the receiver operating characteristic (ROC) curve of the $\mathrm{c} \overline{\mathrm{c}}$ discriminant for identifying a pair of $\mathrm{c}$ quarks from $\mathrm{H}$ boson decay versus quarks from the $\mathrm{V}+$ jets process for large- $R$ jets with $p_{\mathrm{T}}>200 \mathrm{GeV}$ is shown in figure 3 (left). Three working points (WPs) are defined on the $c \overline{\mathrm{c}}$ tagging discriminant distribution with approximately $1,2.5$, and $5 \%$ misidentification rates, and the corresponding efficiencies for identifying a c $\overline{\mathrm{c}}$ pair are approximately 23,35 , and $46 \%$. Another important parameter is the misidentification of $\mathrm{b}$ jets as signal $\mathrm{c}$ jets. The corresponding ROC curve is displayed in figure 3 (right). For the three WPs defined above, the corresponding $\mathrm{b}$ jet misidentification rates are approximately 9,17 , and $27 \%$. To improve the sensitivity of the analysis, three mutually exclusive c $\overline{\mathrm{c}}$-enriched categories, the "low-purity" (LP), "medium-purity" (MP), and "high-purity" (HP) categories, are defined based on the three WPs. The misidentification rate of $\tau$ leptons as signal c jets is larger than the misidentification rate of b jets, but typically a factor of two smaller than the c jet efficiency. However, due to kinematic and mass selection requirements that will be detailed in section 6.2 , the $\mathrm{VH}(\mathrm{H} \rightarrow \tau \tau)$ contribution is much smaller than the $\mathrm{SM} \mathrm{VH}(\mathrm{H} \rightarrow \mathrm{c} \overline{\mathrm{c}})$ signal and hence has a negligible impact on the final result. The merged-jet algorithm is calibrated using data and MC simulated samples. The $p_{\mathrm{T}}$-dependent data-to-simulation scale factors typically range from 0.85 to 1.30, and the corresponding uncertainties range between 20 and $40 \%$.

\subsection{Signal extraction}

In the merged-jet topology analysis, events are required to have at least one large- $R$ jet with $p_{\mathrm{T}}>200 \mathrm{GeV}$, with the highest $p_{\mathrm{T}}$ large- $R$ jet selected as the $\mathrm{H}$ boson candidate. In $\mathrm{VH}(\mathrm{H} \rightarrow \mathrm{c} \overline{\mathrm{c}})$ signal events, the vector boson and the $\mathrm{H}$ boson are typically emitted back-to-back in $\phi$. Therefore, the difference in azimuthal angle between the reconstructed vector boson and $\mathrm{H}$ candidate, $\Delta \phi(\mathrm{V}, \mathrm{H})$, is required to be at least $2.5 \mathrm{rad}$. To avoid double-counting, small- $R$ jets are removed from the event if they overlap the $\mathrm{H}$ candidate with $\Delta R$ (small- $R$ jet, $\mathrm{H})<1.5$. 

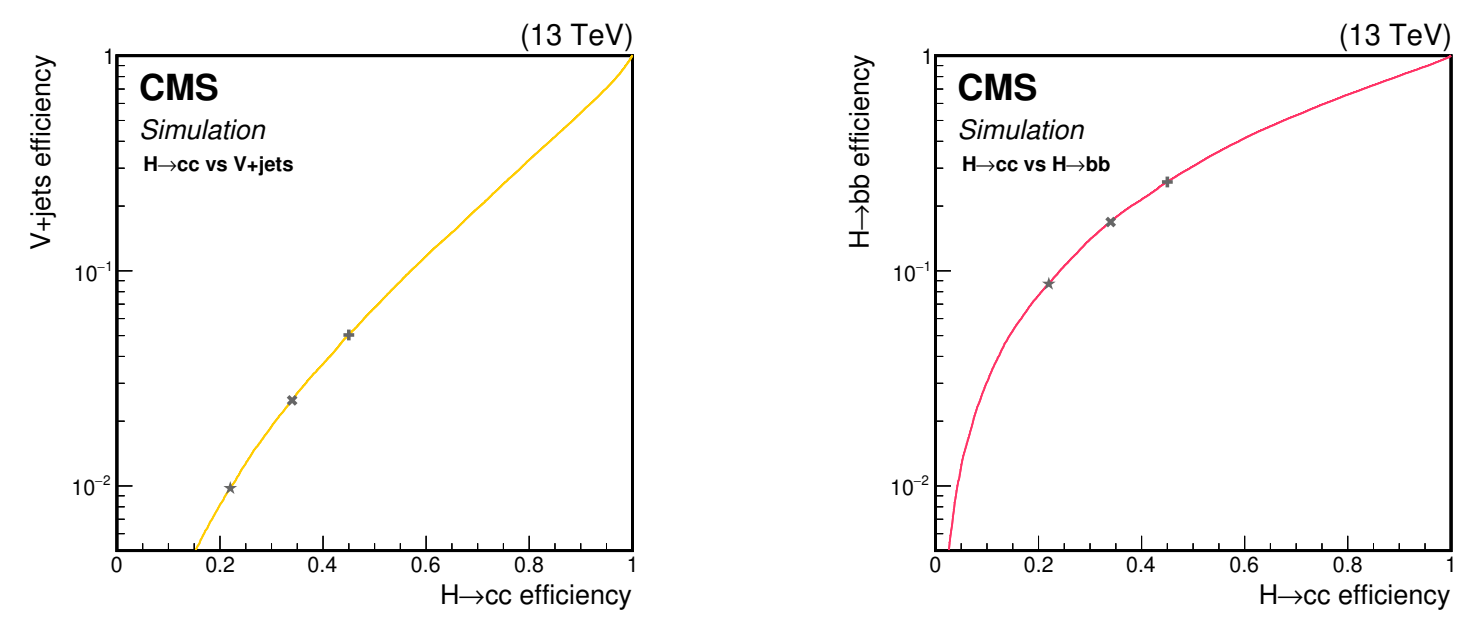

Figure 3. The performance of the $c \bar{c}$ discriminant to identify a $c \bar{c}$ pair in terms of receiver operating characteristic curves, for large- $R$ jets with $p_{\mathrm{T}}>200 \mathrm{GeV}$, before the application of datato-simulation scale factors. Left: the efficiency to correctly identify a pair of c quarks from $\mathrm{H}$ boson decay vs. the efficiency of misidentifying quarks from the $\mathrm{V}+$ jets process. Right: the efficiency to correctly identify a pair of $\mathrm{c}$ quarks from $\mathrm{H}$ boson decay vs. the efficiency of misidentifying a pair of $\mathrm{b}$ quarks from $\mathrm{H}$ boson decay. The gray stars and crosses on the ROC curves represent the three working points used in the merged-jet topology analysis.

To further distinguish the VH signal process from the main backgrounds, a separate BDT is developed for each channel. The goal is to define a discriminant that improves the separation between $\mathrm{VH}$ signal and the main backgrounds, while remaining largely independent of the $c \bar{c}$ tagging discriminant and the $\mathrm{H}$ mass. The BDT only makes use of kinematic information from the event, without including intrinsic properties of $\mathrm{H}$ such as the flavour content and the mass of the large- $R$ jet, which will be used in a fit to the data for the signal extraction. For the signal process, the $\mathrm{VH}(\mathrm{H} \rightarrow \mathrm{b} \overline{\mathrm{b}})$ sample was used instead of the $\mathrm{VH}(\mathrm{H} \rightarrow \mathrm{c} \overline{\mathrm{c}})$ sample, and only events with even event numbers were used to train the BDT while those with odd event numbers were used for the main analysis. As the BDT is designed to be insensitive to the flavour content of the Higgs candidate, training with the $\mathrm{VH}(\mathrm{H} \rightarrow \mathrm{b} \overline{\mathrm{b}})$ signal sample results in no loss of performance. For the background process, only the main backgrounds, e.g., $\mathrm{Z}+$ jets $(\mathrm{V}+$ jets and $\mathrm{t} \overline{\mathrm{t}}+$ jets $)$ in the case of the $2 \mathrm{~L}(0 \mathrm{~L}$ and $1 \mathrm{~L})$ channel are used. Table 2 summarises the kinematic variables used as input to the BDT for each of the three channels.

The BDT distributions of the three channels for events passing the above selection are shown in figure 4 (left) for the $\mathrm{VH}(\mathrm{H} \rightarrow \mathrm{c} \overline{\mathrm{c}}$ ) signal and the background processes. The discrimination power of the BDT depends on the channel. An improved discrimination power is obtained in the $2 \mathrm{~L}$ and $1 \mathrm{~L}$ channels compared to the $0 \mathrm{~L}$ channel. In particular, in the $1 \mathrm{~L}$ channel, improvement is achieved thanks to the presence of the charged lepton and $p_{\mathrm{T}}^{\text {miss }}$, which are then used for the training of the BDT to provide additional handles to suppress the background. For all channels, events with BDT values greater than 0.5 define the signal region. The value of 0.5 was obtained in an optimisation for the $1 \mathrm{~L}$ 


\begin{tabular}{|c|c|c|c|c|}
\hline Variable & Description & $0 \mathrm{~L}$ & $1 \mathrm{~L}$ & $2 \mathrm{~L}$ \\
\hline$p_{\mathrm{T}}(\mathrm{V})$ & vector boson transverse momentum & $\checkmark$ & $\checkmark$ & $\checkmark$ \\
\hline$p_{\mathrm{T}}(\mathrm{H})$ & $\mathrm{H}$ transverse momentum & $\checkmark$ & $\checkmark$ & $\checkmark$ \\
\hline$|\eta(\mathrm{H})|$ & absolute value of the $\mathrm{H}$ pseudorapidity & $\checkmark$ & - & - \\
\hline$\Delta \phi(\mathrm{V}, \mathrm{H})$ & azimuthal angle between vector boson and $\mathrm{H}$ & $\checkmark$ & $\checkmark$ & $\checkmark$ \\
\hline$p_{\mathrm{T}}^{\text {miss }}$ & missing transverse momentum & - & $\checkmark$ & - \\
\hline$\Delta \eta(\mathrm{H}, \ell)$ & difference in pseudorapidity between $\mathrm{H}$ and the lepton & - & $\checkmark$ & - \\
\hline$\Delta \eta(\mathrm{V}, \mathrm{H})$ & difference in pseudorapidity between the vector boson and $\mathrm{H}$ & - & - & $\checkmark$ \\
\hline$\Delta \eta(\mathrm{H}, \mathrm{j})$ & min. difference in pseudorapidity between $\mathrm{H}$ and small- $R$ jets & $\checkmark$ & $\checkmark$ & $\checkmark$ \\
\hline$\Delta \eta(\ell, \mathrm{j})$ & min. difference in pseudorapidity between the lepton and small- $R$ jets & - & $\checkmark$ & - \\
\hline$\Delta \eta(\mathrm{V}, \mathrm{j})$ & min. difference in pseudorapidity between vector boson and small- $R$ jets & - & - & $\checkmark$ \\
\hline$\Delta \phi\left(\vec{p}_{\mathrm{T}}^{\mathrm{miss}}, \mathrm{j}\right)$ & azimuthal angle between $\vec{p}_{\mathrm{T}}^{\text {miss }}$ and closest small- $R$ jet & $\checkmark$ & - & - \\
\hline$\Delta \phi\left(\vec{p}_{\mathrm{T}}^{\mathrm{miss}}, \ell\right)$ & azimuthal angle between $\vec{p}_{\mathrm{T}}^{\text {miss }}$ and lepton & - & $\checkmark$ & - \\
\hline$m_{\mathrm{T}}$ & transverse mass of lepton $\vec{p}_{\mathrm{T}}+\vec{p}_{\mathrm{T}}^{\text {miss }}$ & - & $\checkmark$ & - \\
\hline $\mathrm{N}_{\mathrm{small}-R}^{\mathrm{aj}}$ & number of additional small- $R$ jets & $\checkmark$ & $\checkmark$ & $\checkmark$ \\
\hline
\end{tabular}

Table 2. Variables used in the kinematic BDT training for each channel of the merged-jet topology analysis.

channel, however further tuning of this value for the $0 \mathrm{~L}$ and $2 \mathrm{~L}$ channels has a small impact on the sensitivity. Figure 4 (right) shows the distributions of the $c \bar{c}$ discriminant in the three channels in the signal region for the VH signal and the background processes. Good separation is observed between signal and background. The performance of the $c \bar{c}$ discriminant degrades with the presence of $b$ quarks, as is the case for $t \bar{t}$ events, for instance. The signal regions of the merged-jet topology analysis are finally defined requiring the large- $R$ jet to pass one of the three working points of the $c \bar{c}$ discriminant mentioned above.

Dedicated control regions, each enriched in a specific background process, are defined to aid the background estimation in each channel. Two types of control regions are defined: the "low-BDT" control region consisting of events with BDT value $<0.5$, which is enriched in $\mathrm{V}+$ jets background, and the high $-\mathrm{N}_{\text {small }-R}^{\text {aj }}$ control region, defined by inverting the selection on the number of small- $R$ jets to yield a high-purity t $\bar{t}$ sample. The latter is not used for the 2-lepton channel since the t$\overline{\mathrm{t}}$ contribution is small in this channel. In both control regions, events are required to satisfy the same $c \bar{c}$ tagging discriminant criteria as applied in the signal regions in order to probe events with a similar flavour composition. This allows the efficiency of the above mentioned selection to be estimated directly from the data without further corrections being required, as verified in studies with simulated events and events in data validation regions orthogonal to the control and signal regions.

The low-BDT and the high- $\mathrm{N}_{\text {small- } R}^{\mathrm{aj}}$ control regions, together with the signal regions, are included in the maximum likelihood fit to correct for any difference between data and simulation in the production rate of the $\mathrm{V}+$ jets and $\mathrm{t} \overline{\mathrm{t}}$ processes in the phase space selected by this analysis. Parameters used to separately scale the overall normalisation of the $\mathrm{W}$ + jets, $\mathrm{Z}+$ jets, and $\mathrm{t} \overline{\mathrm{t}}$ background processes are allowed to float freely in the fit. These parameters scale the background rate in the same way in both the control and the signal 

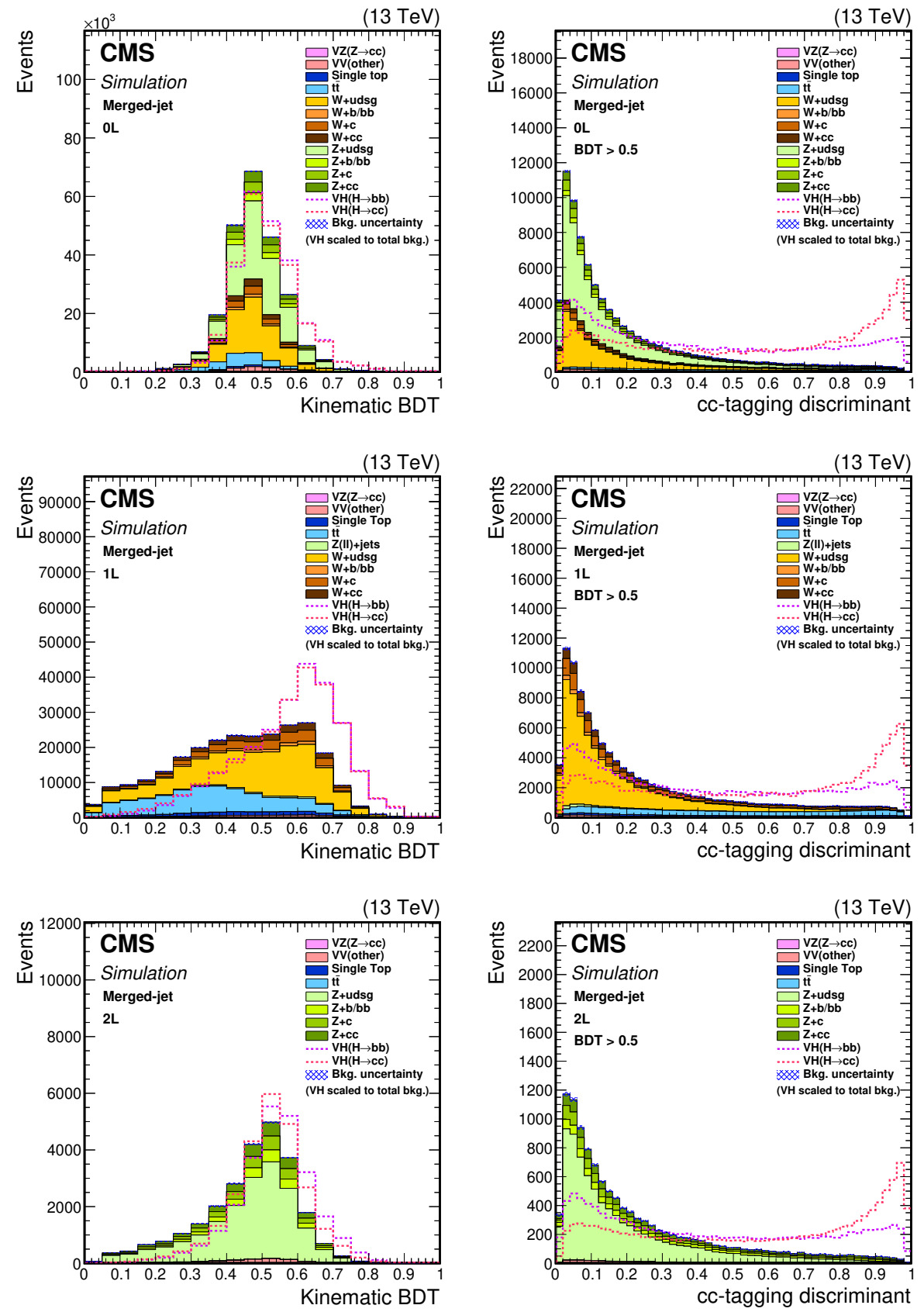

Figure 4. The $\mathrm{VH}(\mathrm{H} \rightarrow \mathrm{c} \overline{\mathrm{c}})$ signal and background distributions of the kinematic BDT output (left), and the $c \bar{c}$ discriminant in events with $\mathrm{BDT}$ values greater than 0.5 (right), in the $0 \mathrm{~L}$ (upper), $1 \mathrm{~L}$ (middle) and $2 \mathrm{~L}$ (lower) channels. The $\mathrm{VH}(\mathrm{H} \rightarrow \mathrm{c} \overline{\mathrm{c}}$ ) signal is normalised to the sum of all backgrounds. The $\mathrm{VH}(\mathrm{H} \rightarrow \mathrm{b} \overline{\mathrm{b}})$ contribution, similarly normalised, is also shown. 
regions. The parameters are defined separately for each channel, with the exception that the same scale factor is assumed for the $\mathrm{W}$ +jets process in the $0 \mathrm{~L}$ and $1 \mathrm{~L}$ channels. Any potential difference in the c $\bar{c}$ tagging efficiency between data and simulation is also taken into account in the measured simulation-to-data scale factors.

The merged-jet topology analysis has been validated in two data samples that are completely independent of the control and signal regions. The first validation sample consists of events with $c \bar{c}$ tagging discriminant values below those used in the control and signal regions, and the second validation sample consists of events with lower $p_{\mathrm{T}}(\mathrm{H})$. Both validation regions count much more data than the signal regions and hence can be helpful in identifying potential issues in the analysis. The outcome of these studies is in good agreement with the SM expectation.

\section{Systematic uncertainties}

Systematic effects can impact the shape of the BDT discriminant distribution for both analysis topologies, as well as the $\mathrm{H}$ candidate mass in the merged-jet topology and the distributions of the charm tagger variables in the resolved-jet topology analysis. The dominant uncertainties are associated with the normalisation of the background from the data control regions and the limited size of the dataset. The values of the rate parameters associated with the background normalisations range from $\sim 0.65$ to $\sim 2.4$ with uncertainties in the range of $10 \%$ to $35 \%$. Additional systematic effects are related to the jet energy scale and resolution, which are treated as correlated between the large- and small- $R$ jets. The efficiency to reconstruct and identify electrons and muons is measured in events with $\mathrm{Z}$ bosons decaying to electrons and muons via the tag and probe method [95]. The uncertainties on these efficiencies range from 2 to $4 \%$. The efficiency for events to pass the combination of triggers based on the presence of one or two leptons is measured using $\mathrm{Z} / \gamma^{*} \rightarrow$ ee and $\mathrm{Z} / \gamma^{*} \rightarrow \mu \mu$ events via the tag-and-probe method [95]. The uncertainties associated with this measurement typically range between 1 and $3 \%$. The efficiency of the $p_{\mathrm{T}}^{\text {miss }}$ trigger is parametrised using a single-muon dataset and the uncertainties are typically below $2 \%$. Theoretical uncertainties related to the cross sections and the $p_{\mathrm{T}}$ spectra of the signal and backgrounds are also considered. In the resolved-jet analysis the systematic uncertainties in PDFs, and in the renormalisation and factorisation scales are treated as uncorrelated among the four flavour classes considered in the $\mathrm{V}+$ jets processes, as described in section 5. The cross section uncertainties are set to their theoretical predictions, while the systematics associated to the renormalisation and factorisation scales are obtained varying the parameters relative to these scales to 0.5 and 2.0 of their nominal values and estimating the effect. Lastly, the uncertainties in the c quark identification are also considered. The full list of systematic uncertainties is provided in table 3 .

In table 4 the uncertainty sources are grouped into categories and their impact on the fitted signal strength resulting from the combination of the resolved-jet and merged-jet topology analyses is provided (see section 8 for more details). The uncertainty breakdown shows that the search for the $\mathrm{VH}(\mathrm{H} \rightarrow \mathrm{c} \overline{\mathrm{c}})$ process is mainly limited by the size of the available dataset: the related uncertainty accounts for more than $85 \%$ of the total uncertainty 


\begin{tabular}{|c|c|c|c|c|}
\hline Source & Type & 0-lepton & 1-lepton & 2-lepton \\
\hline Simulated sample event count & shape & $\checkmark$ & $\checkmark$ & $\checkmark$ \\
\hline c tagging efficiency & shape & $\checkmark$ & $\checkmark$ & $\checkmark$ \\
\hline Top quark $p_{\mathrm{T}}$ reweighting & shape & $\checkmark$ & $\checkmark$ & $\checkmark$ \\
\hline$p_{\mathrm{T}}(\mathrm{V})$ reweighting & shape & $\checkmark$ & $\checkmark$ & $\checkmark$ \\
\hline $\mathrm{VH}: p_{\mathrm{T}}(\mathrm{V})$ NLO EW correction & shape & $\checkmark$ & $\checkmark$ & $\checkmark$ \\
\hline Jet energy scale & shape & $\checkmark$ & $\checkmark$ & $\checkmark$ \\
\hline Jet energy resolution & shape & $\checkmark$ & $\checkmark$ & $\checkmark$ \\
\hline$p_{\mathrm{T}}^{\text {miss }}$ trigger efficiency & rate & $2 \%$ & - & - \\
\hline Lepton trigger efficiency & shape (rate) & - & $\checkmark$ & $\checkmark$ \\
\hline Lepton identification efficiency & shape (rate) & - & $\checkmark$ & $\checkmark$ \\
\hline$p_{\mathrm{T}}^{\text {miss }}$ unclustered energy & shape & $\checkmark$ & $\checkmark$ & - \\
\hline Pileup reweighting & shape & $\checkmark$ & $\checkmark$ & $\checkmark$ \\
\hline Integrated luminosity & rate & $2.5 \%$ & $2.5 \%$ & $2.5 \%$ \\
\hline PDF & shape & $\checkmark$ & $\checkmark$ & $\checkmark$ \\
\hline Renormalisation and factorisation scales & shape & $\checkmark$ & $\checkmark$ & $\checkmark$ \\
\hline Single top cross section & rate & $15 \%$ & $15 \%$ & $15 \%$ \\
\hline Diboson cross section & rate & $10 \%$ & $10 \%$ & $10 \%$ \\
\hline VH: cross section (PDF) & rate & $1.6 \%-2.4 \%$ & $1.9 \%$ & $1.6 \%-2.4 \%$ \\
\hline VH: cross section (scale) & rate & $19 \%-25 \%$ & $0.5 \%-0.7 \%$ & $19 \%-25 \%$ \\
\hline
\end{tabular}

Table 3. Summary of the systematic uncertainties for each channel. The shape uncertainties refer to systematic uncertainties that affect both the shape of the distribution being fitted as well as their normalisation. Uncertainties in the lepton identification and trigger efficiencies are treated as a normalisation uncertainty in the resolved-jet topology analysis and as a shape uncertainty in the merged-jet topology analysis.

in the fitted $\mu$. The statistical uncertainties include contributions from the limited number of events in the available dataset and the background normalisations extracted from the control regions. The main sources of systematic uncertainties come from the charm tagging efficiencies and the modelling of the simulated physics processes, representing $\sim 28 \%$ and $\sim 25 \%$ of the total uncertainty, respectively. The uncertainties in the theory prediction, which include uncertainties in the cross sections, $p_{\mathrm{T}}$ spectra, PDFs, renormalisation and factorisation scales, also play a considerable role and represent approximately $30 \%$ of the total uncertainty in $\mu$.

\section{Results}

The signal extraction strategy is based on a binned likelihood fit to the data, with the signal and control regions fitted simultaneously. Separately fitting electron and muon events in the $1 \mathrm{~L}$ and $2 \mathrm{~L}$ channels is driven by the fact that muons have a significantly smaller misreconstruction rate and greater signal sensitivity. In addition, the trigger, identification and isolation scale factors are different because electrons and muons are reconstructed differently with the CMS detector. The upper limit (UL) on the signal strength $\mu$ for 


\begin{tabular}{|lrr|}
\hline Uncertainty source & $\Delta \mu \mid \mu=37$ \\
\hline Statistical & +17.3 & -17.1 \\
Background normalisations & +10.1 & -10.2 \\
Experimental & +7.6 & -8.2 \\
Charm tagging efficiencies & +5.6 & -4.8 \\
Simulation modeling & +4.2 & -5.1 \\
Jet energy scale and resolution & +2.4 & -2.8 \\
Lepton identification efficiencies & +0.4 & -1.8 \\
Luminosity & +1.6 & -1.7 \\
Statistics of the simulated samples & +0.5 & -1.9 \\
Theory & +6.5 & -4.6 \\
Signal & +5.0 & -2.5 \\
Backgrounds & +4.3 & -3.9 \\
Total & +20.0 & -19.5 \\
\hline
\end{tabular}

Table 4. Summary of the impact of the statistical and systematic uncertainties on the signal strength modifier for combined analysis of the resolved-jet and merged-jet topologies.

$\mathrm{SM}$ production of $\mathrm{VH}(\mathrm{H} \rightarrow c \overline{\mathrm{c}})$ is extracted at 95\% CL based on a modified frequentist approach $[96,97]$ under the asymptotic approximation for the profile likelihood test statistic $[98,99]$. Both analyses are validated by measuring the products of the VZ production cross section and the branching fraction of $\mathrm{Z}$ to charm quark-antiquark pair, $\mathcal{B}(\mathrm{Z} \rightarrow \mathrm{c} \overline{\mathrm{c}})$. The systematic uncertainties are incorporated in the fit as constrained parameters of the likelihood function. The cross section of the $\mathrm{VH}(\mathrm{H} \rightarrow \mathrm{b} \overline{\mathrm{b}})$ process is set to its SM prediction for the $\mathrm{H}$ boson mass of $125 \mathrm{GeV}$. The result has only a weak dependence on the assumed $\mathrm{VH}(\mathrm{H} \rightarrow \mathrm{b} \overline{\mathrm{b}})$ rate. Indeed, on average, the energy carried by neutrinos is higher for the $\mathrm{VH}(\mathrm{H} \rightarrow \mathrm{b} \overline{\mathrm{b}})$ than for the $\mathrm{VH}(\mathrm{H} \rightarrow \mathrm{c} \overline{\mathrm{c}})$ process. This leads to distinguishably different contributions to the final fitted distribution. Varying the $\mathrm{VH}(\mathrm{H} \rightarrow \mathrm{b} \overline{\mathrm{b}})$ rate by $100 \%$ results in less than a $5 \%$ change in the expected sensitivity.

The results obtained in the resolved-jet and merged-jet topology analyses independently, i.e., exploiting larger regions of the full phase space prior to defining disjoint data samples for the combination of results, are described in sections 8.1 and 8.2. As described in sections 5 and 6 , in the merged-jet topology analysis the phase-space considered is bounded from below by $p_{\mathrm{T}}(\mathrm{V})>200 \mathrm{GeV}$, while for the resolved-jet topology analysis the lower bound is set by the $p_{\mathrm{T}}(\mathrm{V})$ thresholds of 50,100 , and $170 \mathrm{GeV}$ in the $2 \mathrm{~L}, 1 \mathrm{~L}$ and 0L channels, respectively. Neither of the analyses has an upper limit on $p_{\mathrm{T}}(\mathrm{V})$. The two analyses are then combined for the final result, presented in section 8.3, after making them statistically independent via a selection on $p_{\mathrm{T}}(\mathrm{V})$ to set an upper bound for the resolved-jet topology analysis that is also the lower bound on the merged-jet topology analysis. 


\subsection{Resolved-jet topology}

In the resolved-jet topology analysis, the $\mathrm{VH}(\mathrm{H} \rightarrow \mathrm{c} \overline{\mathrm{c}})$ signal is extracted via a binned likelihood fit to the BDT output distributions, that is carried out simultaneously with fits to the backgrounds in control regions. In the LF control regions the fits are for the $C v s L_{\text {min }}$ distributions, while in the TT, $\mathrm{HF}$, and $\mathrm{CC}$ control regions they are for the $C v s B_{\text {min }}$ distributions, as detailed in section 5.2.

The analysis is first validated by measuring the product of the VZ production cross section and $\mathcal{B}(\mathrm{Z} \rightarrow \mathrm{c} \overline{\mathrm{c}})$ normalised to the SM prediction. A separate BDT is trained for each channel, using $\mathrm{VZ}(\mathrm{Z} \rightarrow \mathrm{c} \overline{\mathrm{c}})$ as signal and $\mathrm{VH}(\mathrm{H} \rightarrow \mathrm{c} \overline{\mathrm{c}})$ as contribution to background with cross section fixed to the SM prediction. The measured signal strength for the $\mathrm{VZ}(\mathrm{Z} \rightarrow$ $\mathrm{c} \overline{\mathrm{c}})$ process is $\mu_{\mathrm{VZ}(\mathrm{Z} \rightarrow \mathrm{c} \overline{\mathrm{c}})}=1.35_{-0.95}^{+0.94}$ with an observed (expected) significance of 1.5 (1.2) standard deviations $(\sigma)$, respectively. The results are consistent within uncertainties with the SM expectation.

A dedicated BDT is trained for each channel to distinguish the $\mathrm{VH}(\mathrm{H} \rightarrow \mathrm{c} \overline{\mathrm{c}})$ signal from the backgrounds. Figure 5 displays the BDT distributions in all search channels after the fit to the data. In all plots, the value of each nuisance parameter has been fixed to its best fit value. In general, the BDT distributions in data agree well with the background predictions. The largest excess in the data occurs at large BDT values in the high- $p_{\mathrm{T}}(\mathrm{V})$ $2 \mathrm{~L}(\mathrm{Z}(\mathrm{ee}))$ channel with an observed local signal significance of $2.1 \sigma$.

The observed (expected) UL at $95 \% \mathrm{CL}$ on $\mu$ for $\mathrm{SM} \mathrm{VH}(\mathrm{H} \rightarrow \mathrm{c} \overline{\mathrm{c}})$ production is 75 $\left(38_{-11}^{+16}\right)$, and the measured signal strength is $\mu_{\mathrm{VH}(\mathrm{H} \rightarrow \mathrm{c} \overline{\mathrm{c}})}=41_{-20}^{+20}$. The uncertainties in the expected UL correspond to a variation of $\pm 1 \sigma$ in the expected event yields under the background-only hypothesis. The results are consistent with the SM expectations within two standard deviations. This modest deviation is mostly due to the small excess mentioned above. The results for each channel and their combination are shown in table 5 . The most sensitive channel is $2 \mathrm{~L}$, whereas the $0 \mathrm{~L}$ and $1 \mathrm{~L}$ channels have similar sensitivity.

\subsection{Merged-jet topology}

In the merged-jet topology analysis, the $\mathrm{VH}(\mathrm{H} \rightarrow \mathrm{c} \overline{\mathrm{c}})$ signal is extracted via a binned maximum likelihood fit to the soft-drop mass $m_{\mathrm{SD}}$ of $\mathrm{H}$, with the signal regions and the control regions from all three purity categories included in the fit simultaneously. In total, 15 bins are used in the fit for each region, with a bin width of $10 \mathrm{GeV}$ corresponding roughly to the $m_{\mathrm{SD}}$ resolution. The $m_{\mathrm{SD}}$ distributions of the $\mathrm{VH}(\mathrm{H} \rightarrow c \overline{\mathrm{c}})$ and background processes in all three channels in the high-purity category are shown in figure 6 . The background prediction is in good agreement with the observed data, within uncertainties.

Similar to the resolved-jet topology analysis, the full procedure of the merged-jet topology analysis is validated by measuring the product of the VZ production cross section and $\mathcal{B}(\mathrm{Z} \rightarrow \mathrm{c} \overline{\mathrm{c}})$ normalised to the SM prediction. The event selection, including the kinematic $\mathrm{BDT}$, the $c \overline{\mathrm{c}}$ tagging discriminant criteria, and the signal extraction procedure, remain unchanged. In place of $\mathrm{VH}(\mathrm{H} \rightarrow c \overline{\mathrm{c}})$, the $\mathrm{VZ}(\mathrm{Z} \rightarrow \mathrm{c} \overline{\mathrm{c}})$ process is considered to be the signal and $\mathrm{VH}(\mathrm{H} \rightarrow c \bar{c})$ contributes to the background with cross section fixed to the $\mathrm{SM}$ prediction. The measured signal strength is $\mu_{\mathrm{VZ}(\mathrm{Z} \rightarrow \mathrm{c} \overline{\mathrm{c}})}=0.69_{-0.75}^{+0.89}$ with an observed 

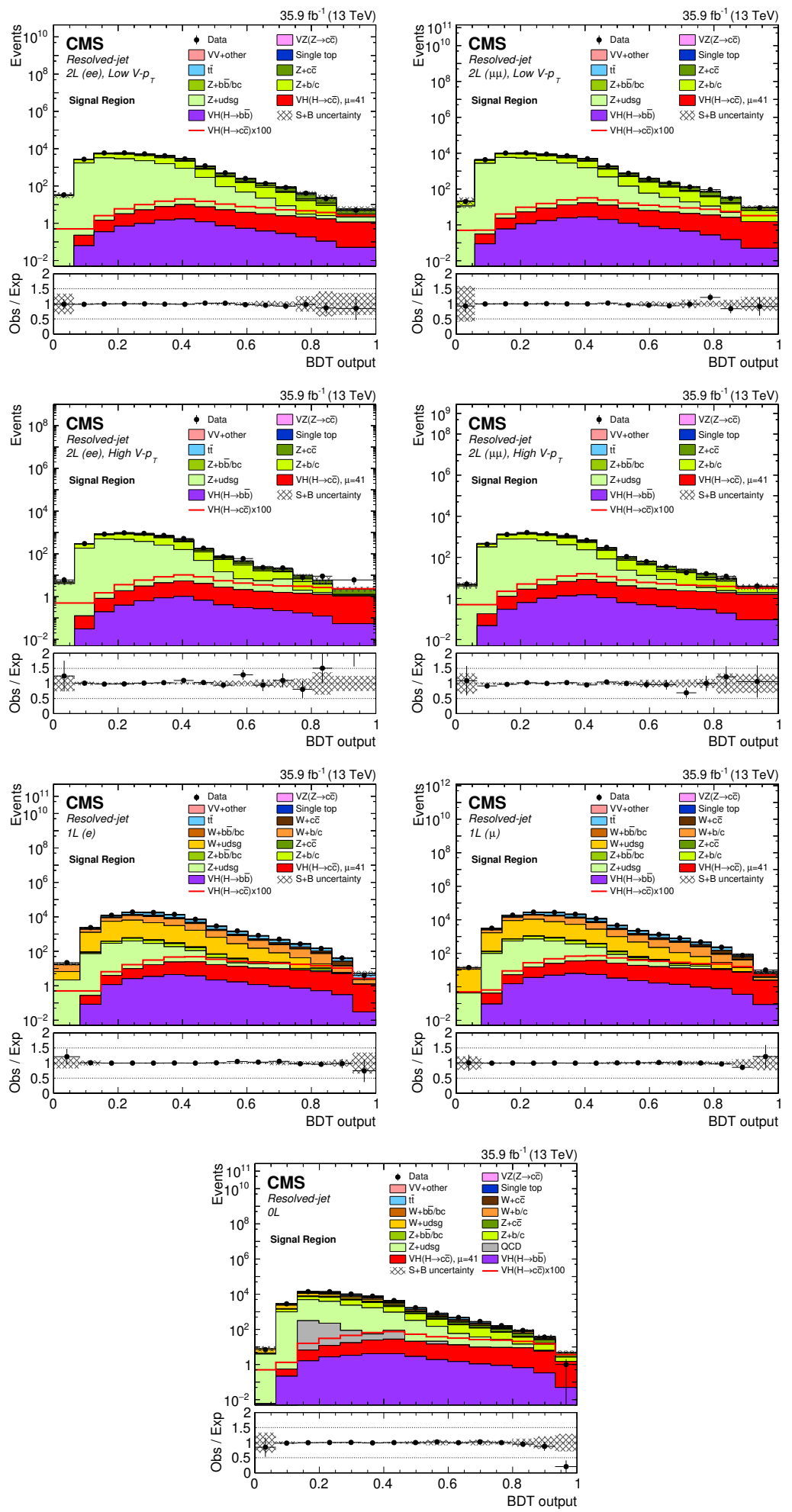

Figure 5. Post-fit distributions of the BDT score in the signal region of the resolved-jet topology analysis for the $2 \mathrm{~L}$ low- $p_{\mathrm{T}}(\mathrm{V}), 2 \mathrm{~L}$ high- $p_{\mathrm{T}}(\mathrm{V}), 1 \mathrm{~L}$, and $0 \mathrm{~L}$ channels. The plain red histograms represent the signal contribution normalized by the post-fit value of $\mu_{\mathrm{VH}(\mathrm{H} \rightarrow \mathrm{c} \overline{\mathrm{c}})}$, while the red line histograms show the expected signal contribution multiplied by a factor 100 . 

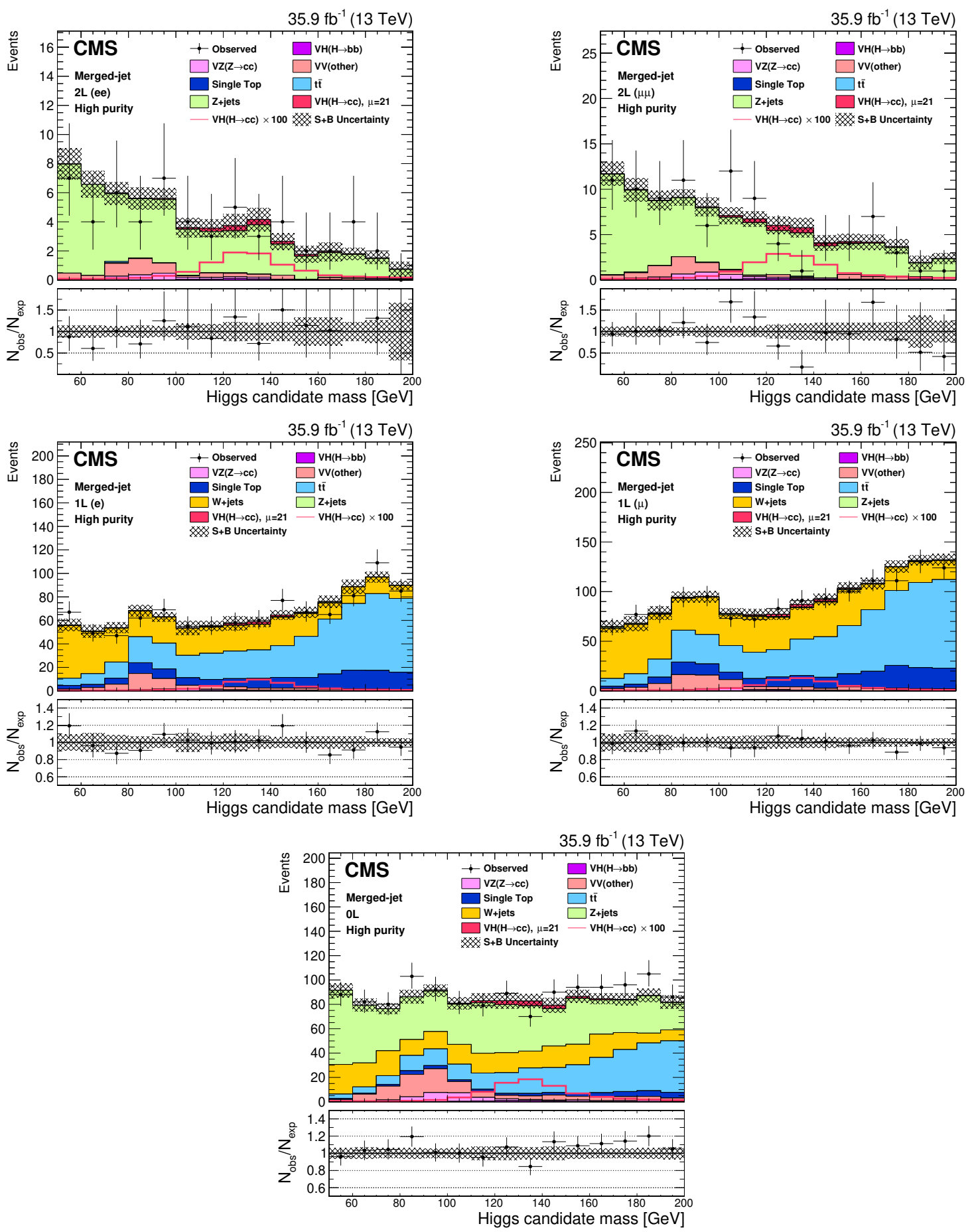

Figure 6. The $m_{\mathrm{SD}}$ distribution of $\mathrm{H}$ in data and simulation in the merged-jet topology analysis signal regions after the maximum likelihood fit, for events in the high purity category. Upper row: 2L channel, electrons (left) and muons (right); middle row: 1L channel, electron (left) and muon (right); lower row: 0L channel. The plain red histograms represent the signal contribution normalized by the post-fit value of $\mu_{\mathrm{VH}(\mathrm{H} \rightarrow \mathrm{c} \overline{\mathrm{c}})}$, while the red line histograms show the expected signal contribution multiplied by a factor 100 . 


\begin{tabular}{|cccccccccc|}
\hline & \multicolumn{4}{c}{ Resolved-jet (inclusive) } & \multicolumn{4}{c|}{ Merged-jet (inclusive) } \\
& $0 \mathrm{~L}$ & $1 \mathrm{~L}$ & $2 \mathrm{~L}$ & All channels & $0 \mathrm{~L}$ & $1 \mathrm{~L}$ & $2 \mathrm{~L}$ & All channels \\
\hline & & & & & & & & \\
Expected UL & $84_{-24}^{+35}$ & $79_{-23}^{+34}$ & $59_{-17}^{+25}$ & $38_{-11}^{+16}$ & $81_{-24}^{+39}$ & $88_{-27}^{+43}$ & $90_{-29}^{+48}$ & $49_{-15}^{+24}$ \\
Observed UL & 66 & 120 & 116 & 75 & 74 & 120 & 76 & 71 \\
\hline
\end{tabular}

Table 5. Observed and expected UL at 95\% CL on the signal strength $\mu$ for the $\mathrm{VH}(\mathrm{H} \rightarrow \mathrm{c} \overline{\mathrm{c}})$ production for the resolved-jet and merged-jet topology analyses, which have a significant overlap. The results are also shown separately for each analysis channel.

(expected) significance of $0.9(1.3) \sigma$. The results are consistent within uncertainties with the SM expectation.

The best fit value of $\mu$ for $\mathrm{SM} \mathrm{VH}(\mathrm{H} \rightarrow \mathrm{c} \overline{\mathrm{c}})$ production is $\mu_{\mathrm{VH}(\mathrm{H} \rightarrow \mathrm{c} \overline{\mathrm{c}})}=21_{-24}^{+26}$, and the observed (expected) UL on $\mu$ is found to be $71\left(49_{-15}^{+24}\right)$ at $95 \%$ CL. The uncertainties in the expected UL correspond to a variation of $\pm 1 \sigma$ in the expected event yields under the background-only hypothesis. The observed values are in agreement with the SM expectation. The results for each channel and their combination are shown in table 5. All channels yield comparable sensitivity in the merged-jet topology analysis.

\subsection{Combination}

To further improve the sensitivity of the search, a single likelihood analysis has been carried out on the two sets of data selected in the merged- and resolved-jet topology analyses. To this end, the two analyses are categorised based on $p_{\mathrm{T}}(\mathrm{V})$. Events with values smaller than a certain value of $p_{\mathrm{T}}(\mathrm{V})$ are used in the resolved-jet topology analysis, whereas the remaining events are used in the merged-jet topology analysis. The main theoretical and experimental systematic uncertainties are correlated between the two analyses, with the exception of those related to the charm tagger efficiency (merged-jet topology) and reshaping (resolved-jet topology), and those related to the $\mathrm{V}+$ jets PDFs and the renormalisation and factorisation scales because of the different treatment of the $\mathrm{V}+$ jets processes adopted for the two analyses. The two regions demarcated by $p_{\mathrm{T}}(\mathrm{V})=300 \mathrm{GeV}$ provide the best combined sensitivity in terms of expected limits on $\mathrm{VH}(\mathrm{H} \rightarrow \mathrm{c} \overline{\mathrm{c}})$.

The combination is validated by measuring the $\mathrm{VZ}(\mathrm{Z} \rightarrow \mathrm{c} \overline{\mathrm{c}})$ signal strength. The measured value is $\mu_{\mathrm{VZ}(\mathrm{Z} \rightarrow \mathrm{c} \overline{\mathrm{c}})}=0.55_{-0.84}^{+0.86}$ with an observed (expected) significance of 0.7 (1.3) $\sigma$.

The fitted $\mathrm{VH}(\mathrm{H} \rightarrow \mathrm{c} \overline{\mathrm{c}})$ signal strength from the combination of the two analyses after the selection on $p_{\mathrm{T}}(\mathrm{V})$ is shown for all channels combined, per production process and per single analysis channel, in figure 7 . The 95\% CL upper limits on the signal strength $\mu_{\mathrm{VH}(\mathrm{H} \rightarrow \mathrm{c} \overline{\mathrm{c}})}$ of each individual analysis after the selection on $p_{\mathrm{T}}(\mathrm{V})$ and their combination is presented in table 6 and displayed in figure 8. The observed (expected) $\mathrm{UL}$ on $\sigma(\mathrm{VH}) \mathcal{B}(\mathrm{H} \rightarrow \mathrm{c} \overline{\mathrm{c}})$ obtained in the combined analysis is $4.5\left(2.4_{-0.7}^{+1.0}\right) \mathrm{pb}$ at $95 \%$ $\mathrm{CL}$, which is equivalent to an observed (expected) upper limit on $\mu$ of $70\left(37_{-11}^{+16}\right)$ at $95 \%$ confidence level. The uncertainties in the expected UL correspond to a variation of $\pm 1 \sigma$ in the expected event yields under the background-only hypothesis. The measured signal strength is $\mu_{\mathrm{VH}(\mathrm{H} \rightarrow \mathrm{c} \overline{\mathrm{c}})}=37_{-17}^{+17}$ (stat) ${ }_{-9}^{+11}$ (syst). The observed values of the signal strength 


\begin{tabular}{|ccccccc|}
\hline \multicolumn{5}{c|}{$95 \%$ CL exclusion limit on $\mu_{\mathrm{VH}(\mathrm{H} \rightarrow \mathrm{c} \overline{\mathrm{c}})}$} \\
& Resolved-jet & Merged-jet & \multicolumn{4}{c|}{ Combination } \\
\hline & $\left(p_{\mathrm{T}}(\mathrm{V})<300 \mathrm{GeV}\right)$ & $\left(p_{\mathrm{T}}(\mathrm{V}) \geq 300 \mathrm{GeV}\right)$ & $0 \mathrm{~L}$ & $1 \mathrm{~L}$ & $2 \mathrm{~L}$ & All channels \\
Expected & $45_{-13}^{+18}$ & $73_{-22}^{+34}$ & $79_{-22}^{+32}$ & $72_{-21}^{+31}$ & $57_{-17}^{+25}$ & $37_{-11}^{+16}$ \\
Observed & 86 & 75 & 83 & 110 & 93 & 70 \\
\hline
\end{tabular}

Table 6. The $95 \% \mathrm{CL}$ upper limits on the signal strength $\mu_{\mathrm{VH}(\mathrm{H} \rightarrow \mathrm{c} \overline{\mathrm{c}})}$ for the $\mathrm{VH}(\mathrm{H} \rightarrow \mathrm{c} \overline{\mathrm{c}})$ process, for the resolved-jet topology analysis for $p_{\mathrm{T}}(\mathrm{V})<300 \mathrm{GeV}$, the merged-jet topology analysis for $p_{\mathrm{T}}(\mathrm{V}) \geq 300 \mathrm{GeV}$, and their combination.

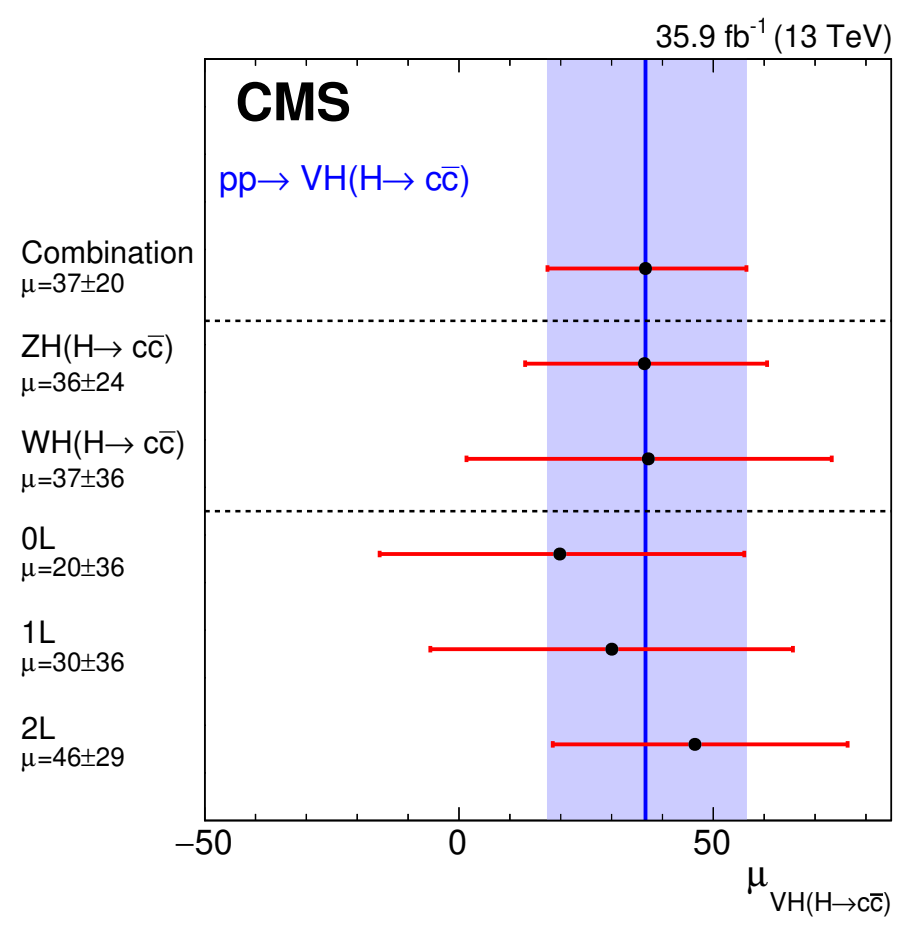

Figure 7. The fitted signal strength $\mu$ for the $\mathrm{ZH}(\mathrm{H} \rightarrow \mathrm{c} \overline{\mathrm{c}})$ and $\mathrm{WH}(\mathrm{H} \rightarrow \mathrm{c} \overline{\mathrm{c}})$ processes, and in each individual channel (0L, 1L, and $2 \mathrm{~L}$ ). The vertical blue line corresponds to the best fit value of $\mu$ for the combination of all channels, while the light-purple band corresponds to the $\pm 1 \sigma$ uncertainty in the measurement.

agrees within $2.1 \sigma$ with the SM expectation. The results in the individual channels also agree with the SM expectation. The modest disagreement between the expected and observed UL's is mainly due to the small excess observed in data in the $\mathrm{Z}(\mathrm{ee})$ high- $p_{\mathrm{T}}(\mathrm{V})$ channel of the resolved-jet topology analysis.

\section{Summary}

In this paper, we present the first search by the CMS Collaboration for the standard model (SM) Higgs boson $\mathrm{H}$ decaying to a pair of charm quarks, produced in association with a vector boson $\mathrm{V}$ ( $\mathrm{W}$ or $\mathrm{Z}$ ). The search uses proton-proton collision data at a centre-of-mass en- 


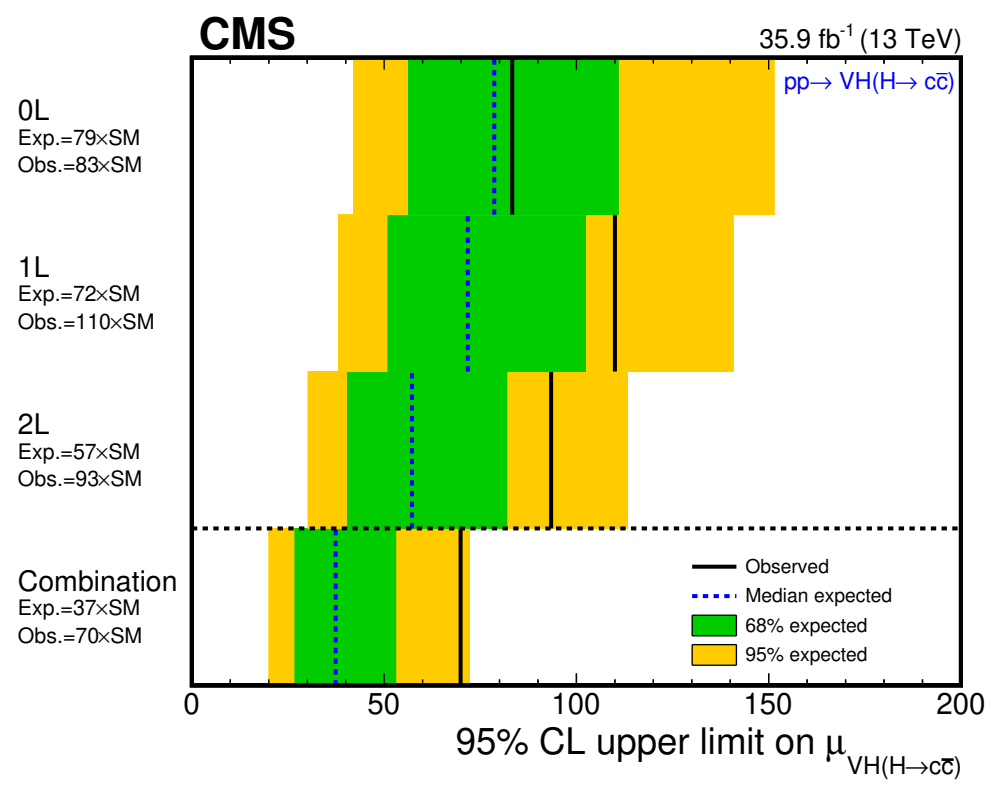

Figure 8. The $95 \%$ CL upper limits on $\mu$ for the $\mathrm{VH}(\mathrm{H} \rightarrow \mathrm{c} \overline{\mathrm{c}})$ process from the combination of the resolved-jet and merged-jet topology analyses in the different channels $(0 \mathrm{~L}, 1 \mathrm{~L}$, and $2 \mathrm{~L})$ and combined. The inner (green) and the outer (yellow) bands indicate the regions containing $68 \%$ and $95 \%$, respectively, of the distribution of limits expected under the background-only hypothesis.

ergy of $13 \mathrm{TeV}$ collected with the CMS detector in 2016 and corresponding to an integrated luminosity of $35.9 \mathrm{fb}^{-1}$. The search is carried out in five modes, $\mathrm{Z}(\mu \mu) \mathrm{H}, \mathrm{Z}(\mathrm{ee}) \mathrm{H}, \mathrm{Z}(v v) \mathrm{H}$, $\mathrm{W}(\mu v) \mathrm{H}$, and $\mathrm{W}(\mathrm{ev}) \mathrm{H}$, with two complementary analyses targeting different regions of phase space. The signal is extracted by statistically combining the results of the two analyses. Each analysis is first validated by carrying out a search for $\mathrm{Z}$ boson decay to a c $\overline{\mathrm{c}}$ pair and comparing the observed signal strength with the SM prediction. The $\mathrm{Z}$ boson signal strength for the combination of the two analyses is measured to be $\mu_{\mathrm{VZ}(\mathrm{Z} \rightarrow \mathrm{c} \overline{\mathrm{c}})}=\sigma / \sigma_{\mathrm{SM}}=$ $0.55_{-0.84}^{+0.86}$, with an observed (expected) significance of 0.7 (1.3) standard deviations.

The measured best fit value of $\sigma(\mathrm{VH}) \mathcal{B}(\mathrm{H} \rightarrow \mathrm{c} \overline{\mathrm{c}})$ for the combination of the two analyses is $2.40_{-1.11}^{+1.12}$ (stat) ${ }_{-0.61}^{+0.65}$ (syst) pb, which corresponds to a best fit value of $\mu$ for $\mathrm{SM} \mathrm{VH}(\mathrm{H} \rightarrow \mathrm{c} \overline{\mathrm{c}})$ production of $\mu_{\mathrm{VH}(\mathrm{H} \rightarrow \mathrm{c} \overline{\mathrm{c}})}=\sigma / \sigma_{\mathrm{SM}}=37_{-17}^{+17}$ (stat) $)_{-9}^{+11}$ (syst), compatible within two standard deviations with the SM prediction. The larger measured $\mu_{\mathrm{VH}(\mathrm{H} \rightarrow \mathrm{c} \overline{\mathrm{c}})}$ value is due to a small excess observed in data in the resolved analysis, with a local significance of 2.1 standard deviations. The observed (expected) 95\% CL upper limit on $\sigma(\mathrm{VH}) \mathcal{B}(\mathrm{H} \rightarrow \mathrm{c} \overline{\mathrm{c}})$ from the combination of the two analyses is $4.5\left(2.4_{-0.7}^{+1.0}\right) \mathrm{pb}$. This limit can be translated into an observed (expected) upper limit on $\mu_{\mathrm{VH}(\mathrm{H} \rightarrow \mathrm{c} \overline{\mathrm{c}})}$ of $70\left(37_{-11}^{+16}\right)$ at $95 \%$ CL by using the theoretical values of the cross section and branching fraction for the SM $\mathrm{H}$ boson with the mass of $125 \mathrm{GeV}$. This result is the most stringent limit on $\sigma(\mathrm{pp} \rightarrow \mathrm{VH}) \mathcal{B}(\mathrm{H} \rightarrow \mathrm{c} \overline{\mathrm{c}})$ to-date. 


\section{Acknowledgments}

We congratulate our colleagues in the CERN accelerator departments for the excellent performance of the LHC and thank the technical and administrative staffs at CERN and at other CMS institutes for their contributions to the success of the CMS effort. In addition, we gratefully acknowledge the computing centres and personnel of the Worldwide LHC Computing Grid for delivering so effectively the computing infrastructure essential to our analyses. Finally, we acknowledge the enduring support for the construction and operation of the LHC and the CMS detector provided by the following funding agencies: BMBWF and FWF (Austria); FNRS and FWO (Belgium); CNPq, CAPES, FAPERJ, FAPERGS, and FAPESP (Brazil); MES (Bulgaria); CERN; CAS, MoST, and NSFC (China); COLCIENCIAS (Colombia); MSES and CSF (Croatia); RPF (Cyprus); SENESCYT (Ecuador); MoER, ERC IUT, PUT and ERDF (Estonia); Academy of Finland, MEC, and HIP (Finland); CEA and CNRS/IN2P3 (France); BMBF, DFG, and HGF (Germany); GSRT (Greece); NKFIA (Hungary); DAE and DST (India); IPM (Iran); SFI (Ireland); INFN (Italy); MSIP and NRF (Republic of Korea); MES (Latvia); LAS (Lithuania); MOE and UM (Malaysia); BUAP, CINVESTAV, CONACYT, LNS, SEP, and UASLP-FAI (Mexico); MOS (Montenegro); MBIE (New Zealand); PAEC (Pakistan); MSHE and NSC (Poland); FCT (Portugal); JINR (Dubna); MON, RosAtom, RAS, RFBR, and NRC KI (Russia); MESTD (Serbia); SEIDI, CPAN, PCTI, and FEDER (Spain); MOSTR (Sri Lanka); Swiss Funding Agencies (Switzerland); MST (Taipei); ThEPCenter, IPST, STAR, and NSTDA (Thailand); TUBITAK and TAEK (Turkey); NASU (Ukraine); STFC (United Kingdom); DOE and NSF (U.S.A.).

Individuals have received support from the Marie-Curie programme and the European Research Council and Horizon 2020 Grant, contract Nos. 675440, 752730, and 765710 (European Union); the Leventis Foundation; the A.P. Sloan Foundation; the Alexander von Humboldt Foundation; the Belgian Federal Science Policy Office; the Fonds pour la Formation à la Recherche dans l'Industrie et dans l'Agriculture (FRIA-Belgium); the Agentschap voor Innovatie door Wetenschap en Technologie (IWT-Belgium); the F.R.S.-FNRS and FWO (Belgium) under the "Excellence of Science - EOS" - be.h project n. 30820817; the Beijing Municipal Science \& Technology Commission, No. Z181100004218003; the Ministry of Education, Youth and Sports (MEYS) of the Czech Republic; the Deutsche Forschungsgemeinschaft (DFG) under Germany's Excellence Strategy — EXC 2121 "Quantum Universe" - 390833306; the Lendület ("Momentum") Programme and the János Bolyai Research Scholarship of the Hungarian Academy of Sciences, the New National Excellence Program ÚNKP, the NKFIA research grants 123842, 123959, 124845, 124850, 125105, 128713, 128786, and 129058 (Hungary); the Council of Science and Industrial Research, India; the HOMING PLUS programme of the Foundation for Polish Science, cofinanced from European Union, Regional Development Fund, the Mobility Plus programme of the Ministry of Science and Higher Education, the National Science Center (Poland), contracts Harmonia 2014/14/M/ST2/00428, Opus 2014/13/B/ST2/02543, 2014/15/B/ST2/03998, and 2015/19/B/ST2/02861, Sonata-bis 2012/07/E/ST2/01406; the National Priorities Research Program by Qatar National Research Fund; the Ministry of Science and Education, 
grant no. 3.2989.2017 (Russia); the Programa Estatal de Fomento de la Investigación Científica y Técnica de Excelencia María de Maeztu, grant MDM-2015-0509 and the Programa Severo Ochoa del Principado de Asturias; the Thalis and Aristeia programmes cofinanced by EU-ESF and the Greek NSRF; the Rachadapisek Sompot Fund for Postdoctoral Fellowship, Chulalongkorn University and the Chulalongkorn Academic into Its 2nd Century Project Advancement Project (Thailand); the Nvidia Corporation; the Welch Foundation, contract C-1845; and the Weston Havens Foundation (U.S.A.).

Open Access. This article is distributed under the terms of the Creative Commons Attribution License (CC-BY 4.0), which permits any use, distribution and reproduction in any medium, provided the original author(s) and source are credited.

\section{References}

[1] ATLAS collaboration, Observation of a new particle in the search for the Standard Model Higgs boson with the ATLAS detector at the LHC, Phys. Lett. B 716 (2012) 1 [arXiv: 1207.7214] [INSPIRE].

[2] CMS collaboration, Observation of a new boson at a mass of $125 \mathrm{GeV}$ with the CMS experiment at the LHC, Phys. Lett. B 716 (2012) 30 [arXiv:1207.7235] [INSPIRE].

[3] CMS collaboration, Observation of a new boson with mass near $125 \mathrm{GeV}$ in pp collisions at $\sqrt{s}=7$ and $8 \mathrm{TeV}$, JHEP 06 (2013) 081 [arXiv:1303.4571] [INSPIRE].

[4] F. Englert and R. Brout, Broken symmetry and the mass of gauge vector mesons, Phys. Rev. Lett. 13 (1964) 321 [INSPIRE].

[5] P.W. Higgs, Broken symmetries and the masses of gauge bosons, Phys. Rev. Lett. 13 (1964) 508 [iNSPIRE].

[6] G.S. Guralnik, C.R. Hagen and T.W.B. Kibble, Global conservation laws and massless particles, Phys. Rev. Lett. 13 (1964) 585 [INSPIRE].

[7] ATLAS and CMS collaborations, Combined measurement of the Higgs boson mass in pp collisions at $\sqrt{s}=7$ and 8 TeV with the ATLAS and CMS experiments, Phys. Rev. Lett. 114 (2015) 191803 [arXiv: 1503.07589] [INSPIRE].

[8] CMS collaboration, Measurements of properties of the Higgs boson decaying into the four-lepton final state in pp collisions at $\sqrt{s}=13$ TeV, JHEP 11 (2017) 047 [arXiv: 1706. 09936] [INSPIRE].

[9] ATLAS collaboration, Measurement of the four-lepton invariant mass spectrum in $13 \mathrm{TeV}$ proton-proton collisions with the ATLAS detector, JHEP 04 (2019) 048 [arXiv: 1902.05892] [INSPIRE].

[10] ATLAS collaboration, Measurement of Higgs boson production in the diphoton decay channel in pp collisions at center-of-mass energies of 7 and $8 \mathrm{TeV}$ with the ATLAS detector, Phys. Rev. D 90 (2014) 112015 [arXiv:1408.7084] [InSPIRE].

[11] CMS collaboration, Observation of the diphoton decay of the Higgs boson and measurement of its properties, Eur. Phys. J. C 74 (2014) 3076 [arXiv: 1407.0558] [INSPIRE].

[12] ATLAS collaboration, Measurements of Higgs boson production and couplings in the four-lepton channel in pp collisions at center-of-mass energies of 7 and $8 \mathrm{TeV}$ with the ATLAS detector, Phys. Rev. D 91 (2015) 012006 [arXiv:1408.5191] [INSPIRE]. 
[13] CMS collaboration, Measurement of the properties of a Higgs boson in the four-lepton final state, Phys. Rev. D 89 (2014) 092007 [arXiv:1312.5353] [InSPIRE].

[14] ATLAS collaboration, Observation and measurement of Higgs boson decays to $W W^{*}$ with the ATLAS detector, Phys. Rev. D 92 (2015) 012006 [arXiv:1412.2641] [INSPIRE].

[15] ATLAS collaboration, Study of $(W / Z) H$ production and Higgs boson couplings using $H \rightarrow W W^{*}$ decays with the ATLAS detector, JHEP 08 (2015) 137 [arXiv:1506.06641] [INSPIRE].

[16] CMS collaboration, Measurement of Higgs boson production and properties in the $W W$ decay channel with leptonic final states, JHEP 01 (2014) 096 [arXiv:1312.1129] [INSPIRE].

[17] ATLAS collaboration, Evidence for the Higgs-boson Yukawa coupling to tau leptons with the ATLAS detector, JHEP 04 (2015) 117 [arXiv: 1501.04943] [INSPIRE].

[18] CMS collaboration, Evidence for the $125 \mathrm{GeV}$ Higgs boson decaying to a pair of $\tau$ leptons, JHEP 05 (2014) 104 [arXiv:1401.5041] [INSPIRE].

[19] CMS collaboration, Observation of the Higgs boson decay to a pair of $\tau$ leptons with the CMS detector, Phys. Lett. B 779 (2018) 283 [arXiv:1708.00373] [InSPIRE].

[20] CMS collaboration, Measurements of properties of the Higgs boson decaying to a $W$ boson pair in pp collisions at $\sqrt{s}=13$ TeV, Phys. Lett. B 791 (2019) 96 [arXiv:1806.05246] [INSPIRE].

[21] CMS collaboration, Measurements of the Higgs boson width and anomalous HVV couplings from on-shell and off-shell production in the four-lepton final state, Phys. Rev. D 99 (2019) 112003 [arXiv: 1901.00174] [INSPIRE].

[22] CMS collaboration, Combined measurements of Higgs boson couplings in proton-proton collisions at $\sqrt{s}=13$ TeV, Eur. Phys. J. C 79 (2019) 421 [arXiv:1809.10733] [INSPIRE].

[23] ATLAS collaboration, Measurements of the Higgs boson production and decay rates and coupling strengths using pp collision data at $\sqrt{s}=7$ and $8 \mathrm{TeV}$ in the ATLAS experiment, Eur. Phys. J. C 76 (2016) 6 [arXiv:1507.04548] [InSPIRE].

[24] CMS collaboration, Precise determination of the mass of the Higgs boson and tests of compatibility of its couplings with the Standard Model predictions using proton collisions at 7 and 8 TeV, Eur. Phys. J. C 75 (2015) 212 [arXiv:1412.8662] [INSPIRE].

[25] CMS collaboration, Study of the mass and spin-parity of the Higgs boson candidate via its decays to $Z$ boson pairs, Phys. Rev. Lett. 110 (2013) 081803 [arXiv:1212.6639] [INSPIRE].

[26] ATLAS collaboration, Evidence for the spin-0 nature of the Higgs boson using ATLAS data, Phys. Lett. B 726 (2013) 120 [arXiv:1307.1432] [INSPIRE].

[27] ATLAS and CMS collaborations, Measurements of the Higgs boson production and decay rates and constraints on its couplings from a combined ATLAS and CMS analysis of the LHC pp collision data at $\sqrt{s}=7$ and $8 \mathrm{TeV}$, JHEP 08 (2016) 045 [arXiv:1606.02266] [INSPIRE].

[28] ATLAS collaboration, Combined measurement of differential and total cross sections in the $H \rightarrow \gamma \gamma$ and the $H \rightarrow Z Z^{*} \rightarrow 4 \ell$ decay channels at $\sqrt{s}=13$ TeV with the ATLAS detector, Phys. Lett. B 786 (2018) 114 [arXiv:1805.10197] [InSPIRE].

[29] ATLAS collaboration, Measurement of the Higgs boson mass in the $H \rightarrow Z Z^{*} \rightarrow 4 \ell$ and $H \rightarrow \gamma \gamma$ channels with $\sqrt{s}=13$ TeV pp collisions using the ATLAS detector, Phys. Lett. B 784 (2018) 345 [arXiv: 1806.00242] [INSPIRE]. 
[30] CMS collaboration, Observation of $t \bar{t} H$ production, Phys. Rev. Lett. 120 (2018) 231801 [arXiv: 1804.02610] [INSPIRE].

[31] ATLAS collaboration, Observation of Higgs boson production in association with a top quark pair at the LHC with the ATLAS detector, Phys. Lett. B 784 (2018) 173 [arXiv: 1806.00425] [INSPIRE].

[32] CMS collaboration, Observation of Higgs boson decay to bottom quarks, Phys. Rev. Lett. 121 (2018) 121801 [arXiv:1808.08242] [INSPIRE].

[33] ATLAS collaboration, Observation of $H \rightarrow b \bar{b}$ decays and $V H$ production with the ATLAS detector, Phys. Lett. B 786 (2018) 59 [arXiv:1808.08238] [INSPIRE].

[34] ATLAS collaboration, A search for the dimuon decay of the Standard Model Higgs boson in pp collisions at $\sqrt{s}=13$ TeV with the ATLAS detector, ATLAS-CONF-2019-028, CERN, Geneva, Switzerland (2019).

[35] CMS collaboration, Search for the Higgs boson decaying to two muons in proton-proton collisions at $\sqrt{s}=13$ TeV, Phys. Rev. Lett. 122 (2019) 021801 [arXiv:1807.06325] [INSPIRE].

[36] C. Delaunay, T. Golling, G. Perez and Y. Soreq, Enhanced Higgs boson coupling to charm pairs, Phys. Rev. D 89 (2014) 033014 [arXiv:1310.7029] [InSPIRE].

[37] D. Ghosh, R.S. Gupta and G. Perez, Is the Higgs mechanism of fermion mass generation a fact? A Yukawa-less first-two-generation model, Phys. Lett. B 755 (2016) 504 [arXiv: 1508.01501] [INSPIRE].

[38] F.J. Botella, G.C. Branco, M.N. Rebelo and J.I. Silva-Marcos, What if the masses of the first two quark families are not generated by the Standard Model Higgs boson?, Phys. Rev. D 94 (2016) 115031 [arXiv:1602.08011] [INSPIRE].

[39] R. Harnik, J. Kopp and J. Zupan, Flavor violating Higgs decays, JHEP 03 (2013) 026 [arXiv: 1209.1397] [INSPIRE].

[40] W. Altmannshofer, J. Eby, S. Gori, M. Lotito, M. Martone and D. Tuckler, Collider signatures of flavorful Higgs bosons, Phys. Rev. D 94 (2016) 115032 [arXiv:1610.02398] [INSPIRE].

[41] G. Perez, Y. Soreq, E. Stamou and K. Tobioka, Constraining the charm Yukawa and Higgs-quark coupling universality, Phys. Rev. D 92 (2015) 033016 [arXiv:1503.00290] [INSPIRE].

[42] LHC Higgs Cross Section Working Group collaboration, Handbook of LHC Higgs cross sections: 4. Deciphering the nature of the Higgs sector, arXiv: 1610.07922 [INSPIRE].

[43] ATLAS collaboration, Search for the decay of the Higgs boson to charm quarks with the ATLAS experiment, Phys. Rev. Lett. 120 (2018) 211802 [arXiv: 1802.04329] [INSPIRE].

[44] CMS collaboration, Identification of heavy-flavour jets with the CMS detector in pp collisions at $13 \mathrm{TeV}, 2018$ JINST 13 P05011 [arXiv: 1712.07158] [INSPIRE].

[45] CMS collaboration, Machine learning-based identification of highly Lorentz-boosted hadronically decaying particles at the CMS experiment, CMS-PAS-JME-18-002, CERN, Geneva, Switzerland (2019).

[46] CMS collaboration, Performance of photon reconstruction and identification with the CMS detector in proton-proton collisions at $\sqrt{s}=8 \mathrm{TeV}, 2015$ JINST $10 \mathrm{P} 08010$ [arXiv: 1502.02702] [INSPIRE]. 
[47] CMS collaboration, Performance of the CMS muon detector and muon reconstruction with proton-proton collisions at $\sqrt{s}=13$ TeV, 2018 JINST 13 P06015 [arXiv:1804.04528] [INSPIRE].

[48] CMS collaboration, The CMS trigger system, 2017 JINST 12 P01020 [arXiv:1609.02366] [INSPIRE].

[49] CMS collaboration, The CMS experiment at the CERN LHC, 2008 JINST 3 S08004 [INSPIRE].

[50] GEANT4 collaboration, GEANT4: a simulation toolkit, Nucl. Instrum. Meth. A 506 (2003) 250 [INSPIRE].

[51] P. Nason, A new method for combining NLO QCD with shower Monte Carlo algorithms, JHEP 11 (2004) 040 [hep-ph/0409146] [INSPIRE].

[52] S. Frixione, P. Nason and C. Oleari, Matching NLO QCD computations with parton shower simulations: the POWHEG method, JHEP 11 (2007) 070 [arXiv:0709.2092] [INSPIRE].

[53] S. Alioli, P. Nason, C. Oleari and E. Re, A general framework for implementing NLO calculations in shower Monte Carlo programs: the POWHEG BOX, JHEP 06 (2010) 043 [arXiv:1002.2581] [INSPIRE].

[54] K. Hamilton, P. Nason and G. Zanderighi, MINLO: Multi-Scale Improved NLO, JHEP 10 (2012) 155 [arXiv:1206.3572] [INSPIRE].

[55] G. Luisoni, P. Nason, C. Oleari and F. Tramontano, $H W^{ \pm} / H Z+0$ and 1 jet at NLO with the POWHEG BOX interfaced to GoSam and their merging within MiNLO, JHEP 10 (2013) 083 [arXiv: 1306.2542] [INSPIRE].

[56] G. Ferrera, M. Grazzini and F. Tramontano, Higher-order QCD effects for associated WH production and decay at the LHC, JHEP 04 (2014) 039 [arXiv: 1312.1669] [INSPIRE].

[57] G. Ferrera, M. Grazzini and F. Tramontano, Associated ZH production at hadron colliders: the fully differential NNLO QCD calculation, Phys. Lett. B 740 (2015) 51 [arXiv: 1407.4747] [INSPIRE].

[58] G. Ferrera, M. Grazzini and F. Tramontano, Associated WH production at hadron colliders: a fully exclusive QCD calculation at NNLO, Phys. Rev. Lett. 107 (2011) 152003 [arXiv:1107.1164] [INSPIRE].

[59] G. Ferrera, G. Somogyi and F. Tramontano, Associated production of a Higgs boson decaying into bottom quarks at the LHC in full NNLO QCD, Phys. Lett. B 780 (2018) 346 [arXiv: 1705.10304] [INSPIRE].

[60] O. Brein, R.V. Harlander and T.J.E. Zirke, vh@nnlo - Higgs strahlung at hadron colliders, Comput. Phys. Commun. 184 (2013) 998 [arXiv:1210.5347] [INSPIRE].

[61] R.V. Harlander, S. Liebler and T. Zirke, Higgs strahlung at the Large Hadron Collider in the 2-Higgs-doublet model, JHEP 02 (2014) 023 [arXiv: 1307.8122] [INSPIRE].

[62] A. Denner, S. Dittmaier, S. Kallweit and A. Mück, HAWK 2.0: a Monte Carlo program for Higgs production in vector-boson fusion and Higgs strahlung at hadron colliders, Comput. Phys. Commun. 195 (2015) 161 [arXiv:1412.5390] [INSPIRE].

[63] J. Alwall et al., The automated computation of tree-level and next-to-leading order differential cross sections and their matching to parton shower simulations, JHEP 07 (2014) 079 [arXiv: 1405.0301] [INSPIRE]. 
[64] Y. Li and F. Petriello, Combining QCD and electroweak corrections to dilepton production in FEWZ, Phys. Rev. D 86 (2012) 094034 [arXiv: 1208.5967] [INSPIRE].

[65] CMS collaboration, Evidence for the Higgs boson decay to a bottom quark-antiquark pair, Phys. Lett. B 780 (2018) 501 [arXiv:1709.07497] [InSPIRE].

[66] S. Kallweit, J.M. Lindert, P. Maierhofer, S. Pozzorini and M. Schönherr, $N L O Q C D+E W$ predictions for $V+$ jets including off-shell vector-boson decays and multijet merging, JHEP 04 (2016) 021 [arXiv: 1511.08692] [INSPIRE].

[67] S. Frixione, P. Nason and G. Ridolfi, A positive-weight next-to-leading-order Monte Carlo for heavy flavour hadroproduction, JHEP 09 (2007) 126 [arXiv:0707.3088] [INSPIRE].

[68] E. Re, Single-top Wt-channel production matched with parton showers using the POWHEG method, Eur. Phys. J. C 71 (2011) 1547 [arXiv: 1009.2450] [INSPIRE].

[69] R. Frederix, E. Re and P. Torrielli, Single-top t-channel hadroproduction in the four-flavour scheme with POWHEG and aMC@NLO, JHEP 09 (2012) 130 [arXiv:1207.5391] [INSPIRE].

[70] S. Alioli, P. Nason, C. Oleari and E. Re, NLO single-top production matched with shower in POWHEG: s- and t-channel contributions, JHEP 09 (2009) 111 [Erratum ibid. 02 (2010) 011] [arXiv: 0907.4076] [INSPIRE].

[71] M. Czakon and A. Mitov, Top++: a program for the calculation of the top-pair cross-section at hadron colliders, Comput. Phys. Commun. 185 (2014) 2930 [arXiv:1112.5675] [INSPIRE].

[72] CMS collaboration, Measurement of differential cross sections for top quark pair production using the lepton+jets final state in proton-proton collisions at $13 \mathrm{TeV}$, Phys. Rev. D 95 (2017) 092001 [arXiv: 1610.04191] [INSPIRE].

[73] NNPDF collaboration, Parton distributions from high-precision collider data, Eur. Phys. J. C 77 (2017) 663 [arXiv:1706.00428] [INSPIRE].

[74] T. Sjöstrand et al., An introduction to PYTHIA 8.2, Comput. Phys. Commun. 191 (2015) 159 [arXiv: 1410.3012] [INSPIRE].

[75] CMS collaboration, Event generator tunes obtained from underlying event and multiparton scattering measurements, Eur. Phys. J. C 76 (2016) 155 [arXiv:1512.00815] [INSPIRE].

[76] R. Frederix and S. Frixione, Merging meets matching in MC@NLO, JHEP 12 (2012) 061 [arXiv: 1209.6215] [INSPIRE].

[77] J. Alwall et al., Comparative study of various algorithms for the merging of parton showers and matrix elements in hadronic collisions, Eur. Phys. J. C 53 (2008) 473 [arXiv:0706.2569] [INSPIRE].

[78] CMS collaboration, Particle-flow reconstruction and global event description with the CMS detector, 2017 JINST 12 P10003 [arXiv:1706. 04965] [INSPIRE].

[79] M. Cacciari, G.P. Salam and G. Soyez, The anti- $k_{t}$ jet clustering algorithm, JHEP 04 (2008) 063 [arXiv: 0802.1189] [INSPIRE].

[80] M. Cacciari, G.P. Salam and G. Soyez, FastJet user manual, Eur. Phys. J. C 72 (2012) 1896 [arXiv: 1111.6097] [INSPIRE].

[81] CMS collaboration, Performance of missing transverse momentum reconstruction in proton-proton collisions at $\sqrt{s}=13$ TeV using the CMS detector, 2019 JINST 14 P07004 [arXiv: 1903.06078] [INSPIRE]. 
[82] CMS collaboration, Performance of electron reconstruction and selection with the CMS detector in proton-proton collisions at $\sqrt{s}=8 \mathrm{TeV}, 2015$ JINST $10 \mathrm{P} 06005$

[arXiv: 1502.02701] [INSPIRE].

[83] CMS collaboration, Pileup mitigation at CMS in 13 TeV data, CMS-PAS-JME-18-001, CERN, Geneva, Switzerland (2019).

[84] CMS collaboration, Jet energy scale and resolution in the CMS experiment in pp collisions at $8 \mathrm{TeV}, 2017$ JINST 12 P02014 [arXiv:1607.03663] [INSPIRE].

[85] CMS collaboration, Jet algorithms performance in 13 TeV data, CMS-PAS-JME-16-003, CERN, Geneva, Switzerland (2017).

[86] CMS collaboration, Pileup removal algorithms, CMS-PAS-JME-14-001, CERN, Geneva, Switzerland (2014).

[87] D. Bertolini, P. Harris, M. Low and N. Tran, Pileup per particle identification, JHEP 10 (2014) 059 [arXiv: 1407.6013] [INSPIRE].

[88] Y.L. Dokshitzer, G.D. Leder, S. Moretti and B.R. Webber, Better jet clustering algorithms, JHEP 08 (1997) 001 [hep-ph/9707323] [INSPIRE].

[89] M. Dasgupta, A. Fregoso, S. Marzani and G.P. Salam, Towards an understanding of jet substructure, JHEP 09 (2013) 029 [arXiv:1307.0007] [INSPIRE].

[90] J.M. Butterworth, A.R. Davison, M. Rubin and G.P. Salam, Jet substructure as a new Higgs search channel at the LHC, Phys. Rev. Lett. 100 (2008) 242001 [arXiv:0802.2470] [INSPIRE].

[91] A.J. Larkoski, S. Marzani, G. Soyez and J. Thaler, Soft drop, JHEP 05 (2014) 146 [arXiv: 1402.2657] [INSPIRE].

[92] A. Hocker et al., TMVA - Toolkit for Multivariate Data Analysis, PoS (ACAT) 040 (2007) [physics/0703039] [INSPIRE].

[93] T. Plehn, G.P. Salam and M. Spannowsky, Fat jets for a light Higgs, Phys. Rev. Lett. 104 (2010) 111801 [arXiv:0910.5472] [INSPIRE].

[94] G. Louppe, M. Kagan and K. Cranmer, Learning to pivot with adversarial networks, in Advances in neural information processing systems 30, I. Guyon et al. eds., Curran Associates Inc., U.S.A. (2017), pg. 981 [arXiv:1611.01046] [INSPIRE].

[95] CMS collaboration, Measurements of inclusive $W$ and $Z$ cross sections in pp collisions at $\sqrt{s}=7 \mathrm{TeV}$, JHEP 01 (2011) 080 [arXiv:1012.2466] [INSPIRE].

[96] A.L. Read, Presentation of search results: the $C L_{s}$ technique, J. Phys. G 28 (2002) 2693 [INSPIRE].

[97] T. Junk, Confidence level computation for combining searches with small statistics, Nucl. Instrum. Meth. A 434 (1999) 435 [hep-ex/9902006] [INSPIRE].

[98] G. Cowan, K. Cranmer, E. Gross and O. Vitells, Asymptotic formulae for likelihood-based tests of new physics, Eur. Phys. J. C 71 (2011) 1554 [Erratum ibid. C 73 (2013) 2501] [arXiv: 1007.1727] [INSPIRE].

[99] ATLAS, CMS collaborations and the LHC Higgs Combination Group, Procedure for the LHC Higgs boson search combination in Summer 2011, CMS-NOTE-2011-005, CERN, Geneva, Switzerland (2011). 


\section{The CMS collaboration}

\section{Yerevan Physics Institute, Yerevan, Armenia}

A.M. Sirunyan ${ }^{\dagger}$, A. Tumasyan

\section{Institut für Hochenergiephysik, Wien, Austria}

W. Adam, F. Ambrogi, T. Bergauer, M. Dragicevic, J. Erö, A. Escalante Del Valle, M. Flechl, R. Frühwirth ${ }^{1}$, M. Jeitler ${ }^{1}$, N. Krammer, I. Krätschmer, D. Liko, T. Madlener, I. Mikulec, N. Rad, J. Schieck ${ }^{1}$, R. Schöfbeck, M. Spanring, D. Spitzbart, W. Waltenberger, C.-E. Wulz ${ }^{1}$, M. Zarucki

Institute for Nuclear Problems, Minsk, Belarus

V. Drugakov, V. Mossolov, J. Suarez Gonzalez

Universiteit Antwerpen, Antwerpen, Belgium

M.R. Darwish, E.A. De Wolf, D. Di Croce, X. Janssen, A. Lelek, M. Pieters, H. Rejeb Sfar, H. Van Haevermaet, P. Van Mechelen, S. Van Putte, N. Van Remortel

\section{Vrije Universiteit Brussel, Brussel, Belgium}

F. Blekman, E.S. Bols, S.S. Chhibra, J. D'Hondt, J. De Clercq, D. Lontkovskyi, S. Lowette, I. Marchesini, S. Moortgat, Q. Python, K. Skovpen, S. Tavernier, W. Van Doninck, P. Van Mulders

\section{Université Libre de Bruxelles, Bruxelles, Belgium}

D. Beghin, B. Bilin, B. Clerbaux, G. De Lentdecker, H. Delannoy, B. Dorney, L. Favart, A. Grebenyuk, A.K. Kalsi, A. Popov, N. Postiau, E. Starling, L. Thomas, C. Vander Velde, P. Vanlaer, D. Vannerom

\section{Ghent University, Ghent, Belgium}

T. Cornelis, D. Dobur, I. Khvastunov², M. Niedziela, C. Roskas, M. Tytgat, W. Verbeke, B. Vermassen, M. Vit

\section{Université Catholique de Louvain, Louvain-la-Neuve, Belgium}

O. Bondu, G. Bruno, C. Caputo, P. David, C. Delaere, M. Delcourt, A. Giammanco, V. Lemaitre, J. Prisciandaro, A. Saggio, M. Vidal Marono, P. Vischia, J. Zobec

\section{Centro Brasileiro de Pesquisas Fisicas, Rio de Janeiro, Brazil}

F.L. Alves, G.A. Alves, G. Correia Silva, C. Hensel, A. Moraes, P. Rebello Teles

Universidade do Estado do Rio de Janeiro, Rio de Janeiro, Brazil

E. Belchior Batista Das Chagas, W. Carvalho, J. Chinellato ${ }^{3}$, E. Coelho, E.M. Da Costa, G.G. Da Silveira ${ }^{4}$, D. De Jesus Damiao, C. De Oliveira Martins, S. Fonseca De Souza, L.M. Huertas Guativa, H. Malbouisson, J. Martins ${ }^{5}$, D. Matos Figueiredo, M. Medina Jaime ${ }^{6}$, M. Melo De Almeida, C. Mora Herrera, L. Mundim, H. Nogima, W.L. Prado Da Silva, L.J. Sanchez Rosas, A. Santoro, A. Sznajder, M. Thiel, E.J. Tonelli Manganote ${ }^{3}$, F. Torres Da Silva De Araujo, A. Vilela Pereira 
Universidade Estadual Paulista ${ }^{a}$, Universidade Federal do $\mathrm{ABC}^{b}$, São Paulo, Brazil

C.A. Bernardes ${ }^{a}$, L. Calligaris ${ }^{a}$, T.R. Fernandez Perez Tomei ${ }^{a}$, E.M. Gregores ${ }^{b}$, D.S. Lemos, P.G. Mercadante ${ }^{b}$, S.F. Novaes ${ }^{a}$, SandraS. Padula ${ }^{a}$

Institute for Nuclear Research and Nuclear Energy, Bulgarian Academy of Sciences, Sofia, Bulgaria

A. Aleksandrov, G. Antchev, R. Hadjiiska, P. Iaydjiev, M. Misheva, M. Rodozov, M. Shopova, G. Sultanov

University of Sofia, Sofia, Bulgaria

M. Bonchev, A. Dimitrov, T. Ivanov, L. Litov, B. Pavlov, P. Petkov

Beihang University, Beijing, China

W. Fang $^{7}$, X. Gao ${ }^{7}$, L. Yuan

Department of Physics, Tsinghua University, Beijing, China

M. Ahmad, Z. Hu, Y. Wang

Institute of High Energy Physics, Beijing, China

G.M. Chen, H.S. Chen, M. Chen, C.H. Jiang, D. Leggat, H. Liao, Z. Liu, A. Spiezia, J. Tao, E. Yazgan, H. Zhang, S. Zhang ${ }^{8}$, J. Zhao

State Key Laboratory of Nuclear Physics and Technology, Peking University, Beijing, China

A. Agapitos, Y. Ban, G. Chen, A. Levin, J. Li, L. Li, Q. Li, Y. Mao, S.J. Qian, D. Wang, Q. Wang

Zhejiang University, Hangzhou, China

M. Xiao

Universidad de Los Andes, Bogota, Colombia

C. Avila, A. Cabrera, C. Florez, C.F. González Hernández, M.A. Segura Delgado

Universidad de Antioquia, Medellin, Colombia

J. Mejia Guisao, J.D. Ruiz Alvarez, C.A. Salazar González, N. Vanegas Arbelaez

University of Split, Faculty of Electrical Engineering, Mechanical Engineering and Naval Architecture, Split, Croatia

D. Giljanović, N. Godinovic, D. Lelas, I. Puljak, T. Sculac

University of Split, Faculty of Science, Split, Croatia

Z. Antunovic, M. Kovac

Institute Rudjer Boskovic, Zagreb, Croatia

V. Brigljevic, D. Ferencek, K. Kadija, B. Mesic, M. Roguljic, A. Starodumov ${ }^{9}$, T. Susa

University of Cyprus, Nicosia, Cyprus

M.W. Ather, A. Attikis, E. Erodotou, A. Ioannou, M. Kolosova, S. Konstantinou,

G. Mavromanolakis, J. Mousa, C. Nicolaou, F. Ptochos, P.A. Razis, H. Rykaczewski,

D. Tsiakkouri 
Charles University, Prague, Czech Republic

M. Finger ${ }^{10}$, M. Finger Jr. ${ }^{10}$, A. Kveton, J. Tomsa

Escuela Politecnica Nacional, Quito, Ecuador

E. Ayala

Universidad San Francisco de Quito, Quito, Ecuador

E. Carrera Jarrin

Academy of Scientific Research and Technology of the Arab Republic of Egypt, Egyptian Network of High Energy Physics, Cairo, Egypt

H. Abdalla ${ }^{11}$, S. Elgammal ${ }^{12}$

National Institute of Chemical Physics and Biophysics, Tallinn, Estonia

S. Bhowmik, A. Carvalho Antunes De Oliveira, R.K. Dewanjee, K. Ehataht, M. Kadastik, M. Raidal, C. Veelken

Department of Physics, University of Helsinki, Helsinki, Finland

P. Eerola, L. Forthomme, H. Kirschenmann, K. Osterberg, M. Voutilainen

Helsinki Institute of Physics, Helsinki, Finland

F. Garcia, J. Havukainen, J.K. Heikkilä, V. Karimäki, M.S. Kim, R. Kinnunen, T. Lampén,

K. Lassila-Perini, S. Laurila, S. Lehti, T. Lindén, P. Luukka, T. Mäenpää, H. Siikonen,

E. Tuominen, J. Tuominiemi

Lappeenranta University of Technology, Lappeenranta, Finland

T. Tuuva

IRFU, CEA, Université Paris-Saclay, Gif-sur-Yvette, France

M. Besancon, F. Couderc, M. Dejardin, D. Denegri, B. Fabbro, J.L. Faure, F. Ferri,

S. Ganjour, A. Givernaud, P. Gras, G. Hamel de Monchenault, P. Jarry, C. Leloup,

B. Lenzi, E. Locci, J. Malcles, J. Rander, A. Rosowsky, M.Ö. Sahin, A. Savoy-Navarro ${ }^{13}$,

M. Titov, G.B. Yu

Laboratoire Leprince-Ringuet, CNRS/IN2P3, Ecole Polytechnique, Institut Polytechnique de Paris

S. Ahuja, C. Amendola, F. Beaudette, P. Busson, C. Charlot, B. Diab, G. Falmagne,

R. Granier de Cassagnac, I. Kucher, A. Lobanov, C. Martin Perez, M. Nguyen, C. Ochando,

P. Paganini, J. Rembser, R. Salerno, J.B. Sauvan, Y. Sirois, A. Zabi, A. Zghiche

Université de Strasbourg, CNRS, IPHC UMR 7178, Strasbourg, France

J.-L. Agram ${ }^{14}$, J. Andrea, D. Bloch, G. Bourgatte, J.-M. Brom, E.C. Chabert, C. Collard,

E. Conte ${ }^{14}$, J.-C. Fontaine ${ }^{14}$, D. Gelé, U. Goerlach, M. Jansová, A.-C. Le Bihan, N. Tonon,

P. Van Hove

Centre de Calcul de l'Institut National de Physique Nucleaire et de Physique des Particules, CNRS/IN2P3, Villeurbanne, France

S. Gadrat 
Université de Lyon, Université Claude Bernard Lyon 1, CNRS-IN2P3, Institut de Physique Nucléaire de Lyon, Villeurbanne, France

S. Beauceron, C. Bernet, G. Boudoul, C. Camen, A. Carle, N. Chanon, R. Chierici, D. Contardo, P. Depasse, H. El Mamouni, J. Fay, S. Gascon, M. Gouzevitch, B. Ille, Sa. Jain, F. Lagarde, I.B. Laktineh, H. Lattaud, A. Lesauvage, M. Lethuillier, L. Mirabito, S. Perries, V. Sordini, L. Torterotot, G. Touquet, M. Vander Donckt, S. Viret

Georgian Technical University, Tbilisi, Georgia

T. Toriashvili ${ }^{15}$

Tbilisi State University, Tbilisi, Georgia

Z. Tsamalaidze ${ }^{10}$

RWTH Aachen University, I. Physikalisches Institut, Aachen, Germany

C. Autermann, L. Feld, K. Klein, M. Lipinski, D. Meuser, A. Pauls, M. Preuten, M.P. Rauch, J. Schulz, M. Teroerde, B. Wittmer

RWTH Aachen University, III. Physikalisches Institut A, Aachen, Germany

M. Erdmann, B. Fischer, S. Ghosh, T. Hebbeker, K. Hoepfner, H. Keller, L. Mastrolorenzo, M. Merschmeyer, A. Meyer, P. Millet, G. Mocellin, S. Mondal, S. Mukherjee, D. Noll, A. Novak, T. Pook, A. Pozdnyakov, T. Quast, M. Radziej, Y. Rath, H. Reithler, J. Roemer, A. Schmidt, S.C. Schuler, A. Sharma, S. Wiedenbeck, S. Zaleski

RWTH Aachen University, III. Physikalisches Institut B, Aachen, Germany G. Flügge, W. Haj Ahmad ${ }^{16}$, O. Hlushchenko, T. Kress, T. Müller, A. Nowack, C. Pistone, O. Pooth, D. Roy, H. Sert, A. Stahl ${ }^{17}$

\section{Deutsches Elektronen-Synchrotron, Hamburg, Germany}

M. Aldaya Martin, P. Asmuss, I. Babounikau, H. Bakhshiansohi, K. Beernaert, O. Behnke, A. Bermúdez Martínez, D. Bertsche, A.A. Bin Anuar, K. Borras ${ }^{18}$, V. Botta, A. Campbell, A. Cardini, P. Connor, S. Consuegra Rodríguez, C. Contreras-Campana, V. Danilov, A. De Wit, M.M. Defranchis, C. Diez Pardos, D. Domínguez Damiani, G. Eckerlin, D. Eckstein, T. Eichhorn, A. Elwood, E. Eren, E. Gallo ${ }^{19}$, A. Geiser, A. Grohsjean, M. Guthoff, M. Haranko, A. Harb, A. Jafari, N.Z. Jomhari, H. Jung, A. Kasem ${ }^{18}$, M. Kasemann, H. Kaveh, J. Keaveney, C. Kleinwort, J. Knolle, D. Krücker, W. Lange, T. Lenz, J. Lidrych, K. Lipka, W. Lohmann ${ }^{20}$, R. Mankel, I.-A. Melzer-Pellmann, A.B. Meyer, M. Meyer, M. Missiroli, J. Mnich, A. Mussgiller, V. Myronenko, D. Pérez Adán, S.K. Pflitsch, D. Pitzl, A. Raspereza, A. Saibel, M. Savitskyi, V. Scheurer, P. Schütze, C. Schwanenberger, R. Shevchenko, A. Singh, H. Tholen, O. Turkot, A. Vagnerini, M. Van De Klundert, R. Walsh, Y. Wen, K. Wichmann, C. Wissing, O. Zenaiev, R. Zlebcik

\section{University of Hamburg, Hamburg, Germany}

R. Aggleton, S. Bein, L. Benato, A. Benecke, V. Blobel, T. Dreyer, A. Ebrahimi, F. Feindt, A. Fröhlich, C. Garbers, E. Garutti, D. Gonzalez, P. Gunnellini, J. Haller, A. Hinzmann, A. Karavdina, G. Kasieczka, R. Klanner, R. Kogler, N. Kovalchuk, S. Kurz, V. Kutzner, J. Lange, T. Lange, A. Malara, J. Multhaup, C.E.N. Niemeyer, A. Perieanu, A. Reimers, 
O. Rieger, C. Scharf, P. Schleper, S. Schumann, J. Schwandt, J. Sonneveld, H. Stadie, G. Steinbrück, F.M. Stober, B. Vormwald, I. Zoi

Karlsruher Institut fuer Technologie, Karlsruhe, Germany

M. Akbiyik, C. Barth, M. Baselga, S. Baur, T. Berger, E. Butz, R. Caspart, T. Chwalek, W. De Boer, A. Dierlamm, K. El Morabit, N. Faltermann, M. Giffels, P. Goldenzweig, A. Gottmann, M.A. Harrendorf, F. Hartmann ${ }^{17}$, U. Husemann, S. Kudella, S. Mitra, M.U. Mozer, D. Müller, Th. Müller, M. Musich, A. Nürnberg, G. Quast, K. Rabbertz, M. Schröder, I. Shvetsov, H.J. Simonis, R. Ulrich, M. Wassmer, M. Weber, C. Wöhrmann, R. Wolf

Institute of Nuclear and Particle Physics (INPP), NCSR Demokritos, Aghia Paraskevi, Greece

G. Anagnostou, P. Asenov, G. Daskalakis, T. Geralis, A. Kyriakis, D. Loukas, G. Paspalaki National and Kapodistrian University of Athens, Athens, Greece

M. Diamantopoulou, G. Karathanasis, P. Kontaxakis, A. Manousakis-katsikakis, A. Panagiotou, I. Papavergou, N. Saoulidou, A. Stakia, K. Theofilatos, K. Vellidis, E. Vourliotis

National Technical University of Athens, Athens, Greece

G. Bakas, K. Kousouris, I. Papakrivopoulos, G. Tsipolitis

University of Ioánnina, Ioánnina, Greece

I. Evangelou, C. Foudas, P. Gianneios, P. Katsoulis, P. Kokkas, S. Mallios, K. Manitara, N. Manthos, I. Papadopoulos, J. Strologas, F.A. Triantis, D. Tsitsonis

MTA-ELTE Lendület CMS Particle and Nuclear Physics Group, Eötvös Loránd University, Budapest, Hungary

M. Bartók ${ }^{21}$, R. Chudasama, M. Csanad, P. Major, K. Mandal, A. Mehta, M.I. Nagy, G. Pasztor, O. Surányi, G.I. Veres

Wigner Research Centre for Physics, Budapest, Hungary

G. Bencze, C. Hajdu, D. Horvath ${ }^{22}$, F. Sikler, T.Á. Vámi, V. Veszpremi, G. Vesztergombi ${ }^{\dagger}$

Institute of Nuclear Research ATOMKI, Debrecen, Hungary

N. Beni, S. Czellar, J. Karancsi ${ }^{21}$, J. Molnar, Z. Szillasi

Institute of Physics, University of Debrecen, Debrecen, Hungary

P. Raics, D. Teyssier, Z.L. Trocsanyi, B. Ujvari

Eszterhazy Karoly University, Karoly Robert Campus, Gyongyos, Hungary

T. Csorgo, W.J. Metzger, F. Nemes, T. Novak

Indian Institute of Science (IISc), Bangalore, India

S. Choudhury, J.R. Komaragiri, P.C. Tiwari

National Institute of Science Education and Research, HBNI, Bhubaneswar, India

S. Bahinipati ${ }^{24}$, C. Kar, G. Kole, P. Mal, V.K. Muraleedharan Nair Bindhu, A. Nayak ${ }^{25}$, D.K. Sahoo ${ }^{24}$, S.K. Swain 
Panjab University, Chandigarh, India

S. Bansal, S.B. Beri, V. Bhatnagar, S. Chauhan, R. Chawla, N. Dhingra, R. Gupta, A. Kaur, M. Kaur, S. Kaur, P. Kumari, M. Lohan, M. Meena, K. Sandeep, S. Sharma, J.B. Singh, A.K. Virdi, G. Walia

University of Delhi, Delhi, India

A. Bhardwaj, B.C. Choudhary, R.B. Garg, M. Gola, S. Keshri, Ashok Kumar, M. Naimuddin, P. Priyanka, K. Ranjan, Aashaq Shah, R. Sharma

Saha Institute of Nuclear Physics, HBNI, Kolkata, India

R. Bhardwaj ${ }^{26}$, M. Bharti ${ }^{26}$, R. Bhattacharya, S. Bhattacharya, U. Bhawandeep ${ }^{26}$,

D. Bhowmik, S. Dutta, S. Ghosh, B. Gomber ${ }^{27}$, M. Maity ${ }^{28}$, K. Mondal, S. Nandan, A. Purohit, P.K. Rout, G. Saha, S. Sarkar, T. Sarkar ${ }^{28}$, M. Sharan, B. Singh ${ }^{26}$, S. Thakur ${ }^{26}$

Indian Institute of Technology Madras, Madras, India

P.K. Behera, P. Kalbhor, A. Muhammad, P.R. Pujahari, A. Sharma, A.K. Sikdar

Bhabha Atomic Research Centre, Mumbai, India

D. Dutta, V. Jha, V. Kumar, D.K. Mishra, P.K. Netrakanti, L.M. Pant, P. Shukla

Tata Institute of Fundamental Research-A, Mumbai, India

T. Aziz, M.A. Bhat, S. Dugad, G.B. Mohanty, N. Sur, RavindraKumar Verma

Tata Institute of Fundamental Research-B, Mumbai, India

S. Banerjee, S. Bhattacharya, S. Chatterjee, P. Das, M. Guchait, S. Karmakar, S. Kumar, G. Majumder, K. Mazumdar, N. Sahoo, S. Sawant

Indian Institute of Science Education and Research (IISER), Pune, India

S. Dube, B. Kansal, A. Kapoor, K. Kothekar, S. Pandey, A. Rane, A. Rastogi, S. Sharma

Institute for Research in Fundamental Sciences (IPM), Tehran, Iran

S. Chenarani ${ }^{29}$, E. Eskandari Tadavani, S.M. Etesami ${ }^{29}$, M. Khakzad, M. Mohammadi Najafabadi, M. Naseri, F. Rezaei Hosseinabadi

University College Dublin, Dublin, Ireland

M. Felcini, M. Grunewald

INFN Sezione di Bari ${ }^{a}$, Università di Bari ${ }^{b}$, Politecnico di Bari ${ }^{c}$, Bari, Italy M. Abbrescia ${ }^{a, b}$, R. Aly ${ }^{a, b, 30}$, C. Calabria ${ }^{a, b}$, A. Colaleo $^{a}$, D. Creanza $^{a, c}$, L. Cristella $^{a, b}$, N. De Filippis ${ }^{a, c}$, M. De Palma ${ }^{a, b}$, A. Di Florio ${ }^{a, b}$, W. Elmetenawee ${ }^{a, b}$, L. Fiore ${ }^{a}$, A. Gelmi ${ }^{a, b}$, G. Iaselli ${ }^{a, c}$, M. Ince ${ }^{a, b}$, S. Lezki ${ }^{a, b}$, G. Maggi ${ }^{a, c}$, M. Maggi ${ }^{a}$, J.A. Merlin, G. Miniello ${ }^{a, b}$, S. My ${ }^{a, b}$, S. Nuzzo ${ }^{a, b}$, A. Pompili ${ }^{a, b}$, G. Pugliese ${ }^{a, c}$, R. Radogna ${ }^{a}$,

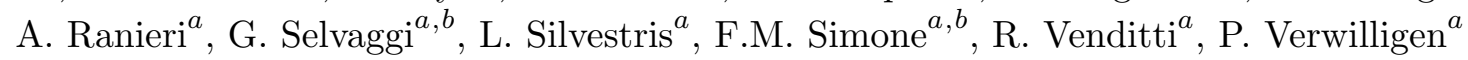

INFN Sezione di Bologna ${ }^{a}$, Università di Bologna ${ }^{b}$, Bologna, Italy

G. Abbiendi ${ }^{a}$, C. Battilana ${ }^{a, b}$, D. Bonacorsi ${ }^{a, b}$, L. Borgonovi $^{a, b}$, S. Braibant-Giacomelli ${ }^{a, b}$,

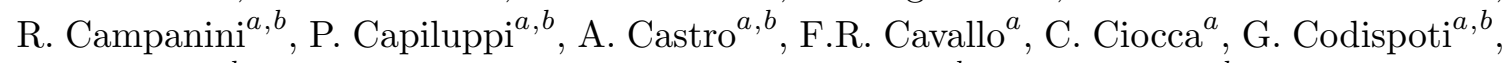

M. Cuffiani ${ }^{a, b}$, G.M. Dallavalle ${ }^{a}$, F. Fabbri ${ }^{a}$, A. Fanfani ${ }^{a, b}$, E. Fontanesi $^{a, b}$, P. Giacomelli $^{a}$,

C. Grandi ${ }^{a}$, L. Guiducci ${ }^{a, b}$, F. Iemmi ${ }^{a, b}$, S. Lo Meo ${ }^{a, 31}$, S. Marcellini ${ }^{a}$, G. Masetti ${ }^{a}$, 


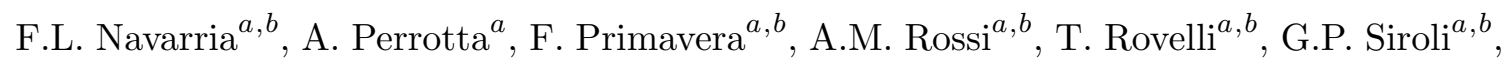
N. $\operatorname{Tosi}^{a}$

INFN Sezione di Catania ${ }^{a}$, Università di Catania ${ }^{b}$, Catania, Italy

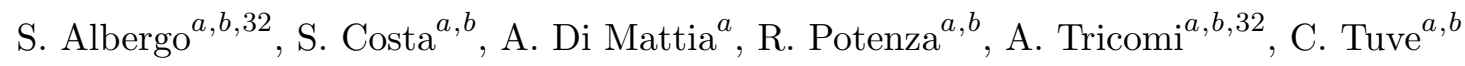

INFN Sezione di Firenze ${ }^{a}$, Università di Firenze ${ }^{b}$, Firenze, Italy

G. Barbagli ${ }^{a}$, A. Cassese, R. Ceccarelli, V. Ciulli ${ }^{a, b}$, C. Civinini ${ }^{a}$, R. D’Alessandro ${ }^{a, b}$,

F. Fiori ${ }^{a, c}$, E. Focardi ${ }^{a, b}$, G. Latino ${ }^{a, b}$, P. Lenzi ${ }^{a, b}$, M. Meschini ${ }^{a}$, S. Paoletti ${ }^{a}$,

G. Sguazzoni ${ }^{a}$, L. Viliani ${ }^{a}$

INFN Laboratori Nazionali di Frascati, Frascati, Italy

L. Benussi, S. Bianco, D. Piccolo

INFN Sezione di Genova ${ }^{a}$, Università di Genova ${ }^{b}$, Genova, Italy

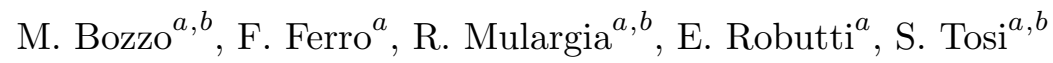

INFN Sezione di Milano-Bicocca ${ }^{a}$, Università di Milano-Bicocca ${ }^{b}$, Milano, Italy

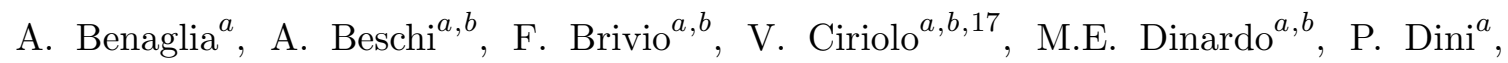

S. Gennai ${ }^{a}$, A. Ghezzi ${ }^{a, b}$, P. Govoni ${ }^{a, b}$, L. Guzzi ${ }^{a, b}$, M. Malberti ${ }^{a}$, S. Malvezzi ${ }^{a}$,

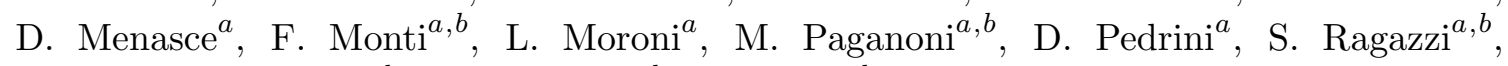

T. Tabarelli de Fatis ${ }^{a, b}$, D. Valsecchi ${ }^{a, b}$, D. Zuolo ${ }^{a, b}$

INFN Sezione di Napoli ${ }^{a}$, Università di Napoli 'Federico II' ${ }^{b}$, Napoli, Italy, Università della Basilicata $^{c}$, Potenza, Italy, Università G. Marconi ${ }^{d}$, Roma, Italy

S. Buontempo ${ }^{a}$, N. Cavallo ${ }^{a, c}$, A. De Iorio ${ }^{a, b}$, A. Di Crescenzo ${ }^{a, b}$, F. Fabozzi $^{a, c}$, F. Fienga $^{a}$,

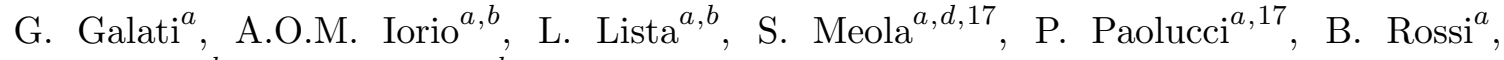
C. Sciacca ${ }^{a, b}$, E. Voevodina ${ }^{a, b}$

INFN Sezione di Padova ${ }^{a}$, Università di Padova ${ }^{b}$, Padova, Italy, Università di Trento ${ }^{c}$, Trento, Italy

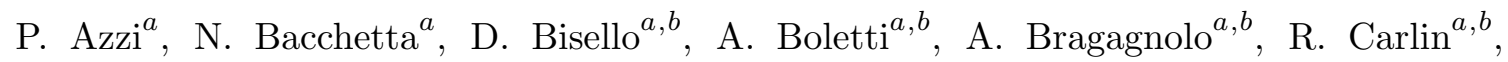

P. Checchia ${ }^{a}$, P. De Castro Manzano ${ }^{a}$, T. Dorigo ${ }^{a}$, U. Dosselli ${ }^{a}$, F. Gasparini ${ }^{a, b}{ }^{\prime}$

U. Gasparini ${ }^{a, b}$, A. Gozzelino ${ }^{a}$, S.Y. Hoh $^{a, b}$, P. Lujan ${ }^{a}$, M. Margoni $^{a, b}$, A.T. Meneguzzo ${ }^{a, b}$,

J. Pazzini ${ }^{a, b}$, M. Presilla ${ }^{b}$, P. Ronchese ${ }^{a, b}$, R. Rossin ${ }^{a, b}$, F. Simonetto ${ }^{a, b}$, A. Tiko ${ }^{a}$, M. Tosi ${ }^{a, b}$, M. Zanetti ${ }^{a, b}$, P. Zotto ${ }^{a, b}$, G. Zumerle ${ }^{a, b}$

INFN Sezione di Pavia ${ }^{a}$, Università di Pavia ${ }^{b}$, Pavia, Italy

A. Braghieri ${ }^{a}$, D. Fiorina ${ }^{a, b}$, P. Montagna ${ }^{a, b}$, S.P. Ratti ${ }^{a, b}$, V. $\operatorname{Re}^{a}$, M. Ressegotti ${ }^{a, b}$, C. Riccardi ${ }^{a, b}$, P. Salvini ${ }^{a}$, I. Vai $^{a}$, P. Vitulo ${ }^{a, b}$

INFN Sezione di Perugia ${ }^{a}$, Università di Perugia ${ }^{b}$, Perugia, Italy

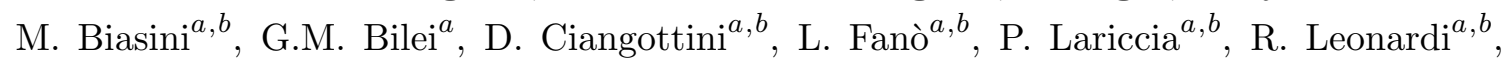

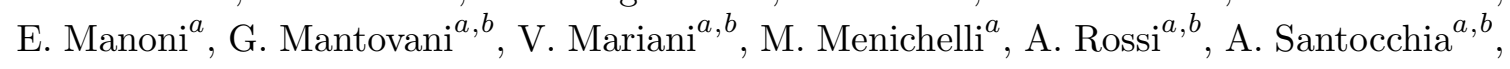

D. Spiga ${ }^{a}$ 
INFN Sezione di Pisa ${ }^{a}$, Università di Pisa ${ }^{b}$, Scuola Normale Superiore di Pisa c, Pisa, Italy

K. Androsov $^{a}$, P. Azzurri ${ }^{a}$, G. Bagliesi ${ }^{a}$, V. Bertacchi ${ }^{a, c}$, L. Bianchini $^{a}$, T. Boccali ${ }^{a}$,

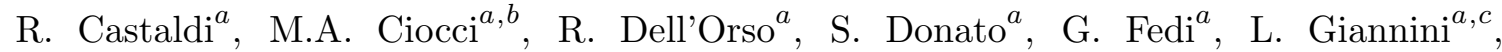

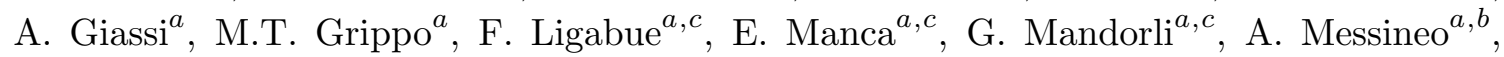
F. Palla ${ }^{a}$, A. Rizzi ${ }^{a}, b$, G. Rolandi ${ }^{33}$, S. Roy Chowdhury, A. Scribano ${ }^{a}$, P. Spagnolo ${ }^{a}$,

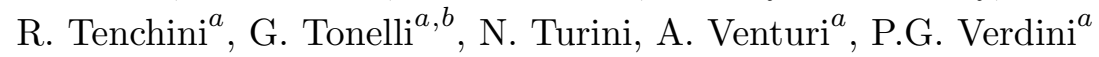

INFN Sezione di Roma ${ }^{a}$, Sapienza Università di Roma ${ }^{b}$, Rome, Italy

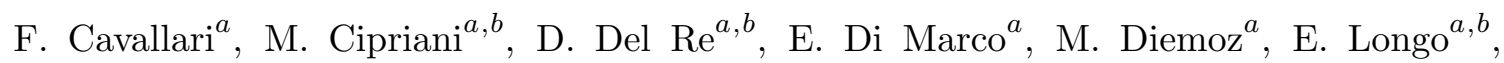
P. Meridiani ${ }^{a}$, G. Organtini ${ }^{a, b}$, F. Pandolfi ${ }^{a}$, R. Paramatti ${ }^{a, b}$, C. Quaranta ${ }^{a, b}$, S. Rahatlou ${ }^{a, b}$, C. Rovelli ${ }^{a}$, F. Santanastasio ${ }^{a, b}$, L. Soffi $^{a, b}$

INFN Sezione di Torino ${ }^{a}$, Università di Torino ${ }^{b}$, Torino, Italy, Università del Piemonte Orientale ${ }^{c}$, Novara, Italy

N. Amapane ${ }^{a, b}$, R. Arcidiacono ${ }^{a, c}, \mathrm{~S}$. Argiro ${ }^{a, b}$, M. Arneodo $^{a, c}$, N. Bartosik ${ }^{a}$, R. Bellan $^{a, b}$, A. Bellora, C. Biino ${ }^{a}$, A. Cappati ${ }^{a, b}$, N. Cartiglia ${ }^{a}$, S. Cometti ${ }^{a}$, M. Costa ${ }^{a, b}$, R. Covarelli ${ }^{a, b}$, N. Demaria ${ }^{a}$, B. Kiani ${ }^{a, b}$, F. Legger, C. Mariotti ${ }^{a}$, S. Maselli ${ }^{a}$,

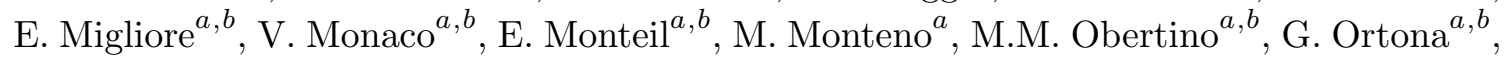
L. Pacher ${ }^{a, b}$, N. Pastrone ${ }^{a}$, M. Pelliccioni ${ }^{a}$, G.L. Pinna Angioni ${ }^{a, b}$, A. Romero ${ }^{a, b}$,

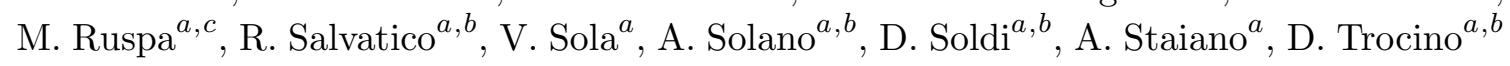
INFN Sezione di Trieste ${ }^{a}$, Università di Trieste ${ }^{b}$, Trieste, Italy

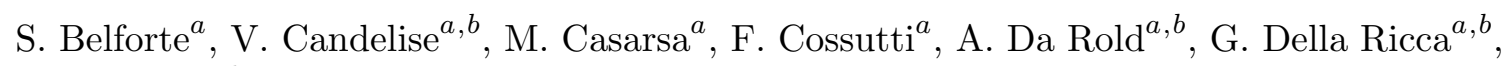
F. Vazzoler ${ }^{a, b}$, A. Zanetti ${ }^{a}$

Kyungpook National University, Daegu, Korea

B. Kim, D.H. Kim, G.N. Kim, J. Lee, S.W. Lee, C.S. Moon, Y.D. Oh, S.I. Pak, S. Sekmen, D.C. Son, Y.C. Yang

Chonnam National University, Institute for Universe and Elementary Particles, Kwangju, Korea

H. Kim, D.H. Moon, G. Oh

Hanyang University, Seoul, Korea

B. Francois, T.J. Kim, J. Park

Korea University, Seoul, Korea

S. Cho, S. Choi, Y. Go, S. Ha, B. Hong, K. Lee, K.S. Lee, J. Lim, J. Park, S.K. Park, Y. Roh, J. Yoo

Kyung Hee University, Department of Physics

J. Goh

Sejong University, Seoul, Korea

H.S. Kim 
Seoul National University, Seoul, Korea

J. Almond, J.H. Bhyun, J. Choi, S. Jeon, J. Kim, J.S. Kim, H. Lee, K. Lee, S. Lee, K. Nam, M. Oh, S.B. Oh, B.C. Radburn-Smith, U.K. Yang, H.D. Yoo, I. Yoon

University of Seoul, Seoul, Korea

D. Jeon, J.H. Kim, J.S.H. Lee, I.C. Park, I.J Watson

Sungkyunkwan University, Suwon, Korea

Y. Choi, C. Hwang, Y. Jeong, J. Lee, Y. Lee, I. Yu

Riga Technical University, Riga, Latvia

V. Veckalns ${ }^{34}$

Vilnius University, Vilnius, Lithuania

V. Dudenas, A. Juodagalvis, A. Rinkevicius, G. Tamulaitis, J. Vaitkus

National Centre for Particle Physics, Universiti Malaya, Kuala Lumpur, Malaysia

Z.A. Ibrahim, F. Mohamad Idris ${ }^{35}$, W.A.T. Wan Abdullah, M.N. Yusli, Z. Zolkapli

Universidad de Sonora (UNISON), Hermosillo, Mexico

J.F. Benitez, A. Castaneda Hernandez, J.A. Murillo Quijada, L. Valencia Palomo

Centro de Investigacion y de Estudios Avanzados del IPN, Mexico City, Mexico

H. Castilla-Valdez, E. De La Cruz-Burelo, I. Heredia-De La Cruz ${ }^{36}$, R. Lopez-Fernandez,

A. Sanchez-Hernandez

Universidad Iberoamericana, Mexico City, Mexico

S. Carrillo Moreno, C. Oropeza Barrera, M. Ramirez-Garcia, F. Vazquez Valencia

Benemerita Universidad Autonoma de Puebla, Puebla, Mexico

J. Eysermans, I. Pedraza, H.A. Salazar Ibarguen, C. Uribe Estrada

Universidad Autónoma de San Luis Potosí, San Luis Potosí, Mexico

A. Morelos Pineda

University of Montenegro, Podgorica, Montenegro

J. Mijuskovic ${ }^{2}$, N. Raicevic

University of Auckland, Auckland, New Zealand

D. Krofcheck

University of Canterbury, Christchurch, New Zealand

S. Bheesette, P.H. Butler

National Centre for Physics, Quaid-I-Azam University, Islamabad, Pakistan

A. Ahmad, M. Ahmad, Q. Hassan, H.R. Hoorani, W.A. Khan, M.A. Shah, M. Shoaib, M. Waqas

AGH University of Science and Technology Faculty of Computer Science, Electronics and Telecommunications, Krakow, Poland

V. Avati, L. Grzanka, M. Malawski 
National Centre for Nuclear Research, Swierk, Poland

H. Bialkowska, M. Bluj, B. Boimska, M. Górski, M. Kazana, M. Szleper, P. Zalewski

Institute of Experimental Physics, Faculty of Physics, University of Warsaw, Warsaw, Poland

K. Bunkowski, A. Byszuk ${ }^{37}$, K. Doroba, A. Kalinowski, M. Konecki, J. Krolikowski, M. Olszewski, M. Walczak

Laboratório de Instrumentação e Física Experimental de Partículas, Lisboa, Portugal

M. Araujo, P. Bargassa, D. Bastos, A. Di Francesco, P. Faccioli, B. Galinhas, M. Gallinaro,

J. Hollar, N. Leonardo, T. Niknejad, J. Seixas, K. Shchelina, G. Strong, O. Toldaiev,

J. Varela

Joint Institute for Nuclear Research, Dubna, Russia

S. Afanasiev, P. Bunin, M. Gavrilenko, I. Golutvin, I. Gorbunov, A. Kamenev, V. Karjavine, A. Lanev, A. Malakhov, V. Matveev ${ }^{38,39}$, P. Moisenz, V. Palichik, V. Perelygin, M. Savina, S. Shmatov, S. Shulha, N. Skatchkov, V. Smirnov, N. Voytishin, A. Zarubin

Petersburg Nuclear Physics Institute, Gatchina (St. Petersburg), Russia

L. Chtchipounov, V. Golovtcov, Y. Ivanov, V. Kim ${ }^{40}$, E. Kuznetsova ${ }^{41}$, P. Levchenko, V. Murzin, V. Oreshkin, I. Smirnov, D. Sosnov, V. Sulimov, L. Uvarov, A. Vorobyev

Institute for Nuclear Research, Moscow, Russia

Yu. Andreev, A. Dermenev, S. Gninenko, N. Golubev, A. Karneyeu, M. Kirsanov, N. Krasnikov, A. Pashenkov, D. Tlisov, A. Toropin

Institute for Theoretical and Experimental Physics named by A.I. Alikhanov of NRC 'Kurchatov Institute', Moscow, Russia

V. Epshteyn, V. Gavrilov, N. Lychkovskaya, A. Nikitenko ${ }^{42}$, V. Popov, I. Pozdnyakov, G. Safronov, A. Spiridonov, A. Stepennov, M. Toms, E. Vlasov, A. Zhokin

Moscow Institute of Physics and Technology, Moscow, Russia

T. Aushev

National Research Nuclear University 'Moscow Engineering Physics Institute' (MEPhI), Moscow, Russia

O. Bychkova, R. Chistov ${ }^{43}$, M. Danilov ${ }^{43}$, S. Polikarpov ${ }^{43}$, E. Tarkovskii

P.N. Lebedev Physical Institute, Moscow, Russia

V. Andreev, M. Azarkin, I. Dremin, M. Kirakosyan, A. Terkulov

Skobeltsyn Institute of Nuclear Physics, Lomonosov Moscow State University, Moscow, Russia

A. Baskakov, A. Belyaev, E. Boos, V. Bunichev, M. Dubinin ${ }^{44}$, L. Dudko, V. Klyukhin,

O. Kodolova, I. Lokhtin, S. Obraztsov, M. Perfilov, S. Petrushanko, V. Savrin

Novosibirsk State University (NSU), Novosibirsk, Russia

A. Barnyakov ${ }^{45}$, V. Blinov ${ }^{45}$, T. Dimova ${ }^{45}$, L. Kardapoltsev ${ }^{45}$, Y. Skovpen ${ }^{45}$ 
Institute for High Energy Physics of National Research Centre 'Kurchatov Institute', Protvino, Russia

I. Azhgirey, I. Bayshev, S. Bitioukov, V. Kachanov, D. Konstantinov, P. Mandrik, V. Petrov, R. Ryutin, S. Slabospitskii, A. Sobol, S. Troshin, N. Tyurin, A. Uzunian, A. Volkov

National Research Tomsk Polytechnic University, Tomsk, Russia

A. Babaev, A. Iuzhakov, V. Okhotnikov

Tomsk State University, Tomsk, Russia

V. Borchsh, V. Ivanchenko, E. Tcherniaev

University of Belgrade: Faculty of Physics and VINCA Institute of Nuclear Sciences

P. Adzic ${ }^{46}$, P. Cirkovic, M. Dordevic, P. Milenovic, J. Milosevic, M. Stojanovic

Centro de Investigaciones Energéticas Medioambientales y Tecnológicas (CIEMAT), Madrid, Spain

M. Aguilar-Benitez, J. Alcaraz Maestre, A. Álvarez Fernández, I. Bachiller, M. Barrio Luna, CristinaF. Bedoya, J.A. Brochero Cifuentes, C.A. Carrillo Montoya, M. Cepeda, M. Cerrada, N. Colino, B. De La Cruz, A. Delgado Peris, J.P. Fernández Ramos, J. Flix, M.C. Fouz, O. Gonzalez Lopez, S. Goy Lopez, J.M. Hernandez, M.I. Josa, D. Moran, Á. Navarro Tobar, A. Pérez-Calero Yzquierdo, J. Puerta Pelayo, I. Redondo, L. Romero, S. Sánchez Navas, M.S. Soares, A. Triossi, C. Willmott

Universidad Autónoma de Madrid, Madrid, Spain

C. Albajar, J.F. de Trocóniz, R. Reyes-Almanza

Universidad de Oviedo, Instituto Universitario de Ciencias y Tecnologías Espaciales de Asturias (ICTEA), Oviedo, Spain

B. Alvarez Gonzalez, J. Cuevas, C. Erice, J. Fernandez Menendez, S. Folgueras, I. Gonzalez Caballero, J.R. González Fernández, E. Palencia Cortezon, V. Rodríguez Bouza, S. Sanchez Cruz

Instituto de Física de Cantabria (IFCA), CSIC-Universidad de Cantabria, Santander, Spain

I.J. Cabrillo, A. Calderon, B. Chazin Quero, J. Duarte Campderros, M. Fernandez, P.J. Fernández Manteca, A. García Alonso, G. Gomez, C. Martinez Rivero, P. Martinez Ruiz del Arbol, F. Matorras, J. Piedra Gomez, C. Prieels, T. Rodrigo, A. Ruiz-Jimeno, L. Russo $^{47}$, L. Scodellaro, I. Vila, J.M. Vizan Garcia

University of Colombo, Colombo, Sri Lanka

K. Malagalage

University of Ruhuna, Department of Physics, Matara, Sri Lanka

W.G.D. Dharmaratna, N. Wickramage 


\section{CERN, European Organization for Nuclear Research, Geneva, Switzerland}

D. Abbaneo, B. Akgun, E. Auffray, G. Auzinger, J. Baechler, P. Baillon, A.H. Ball,

D. Barney, J. Bendavid, M. Bianco, A. Bocci, P. Bortignon, E. Bossini, E. Brondolin,

T. Camporesi, A. Caratelli, G. Cerminara, E. Chapon, G. Cucciati, D. d'Enterria,

A. Dabrowski, N. Daci, V. Daponte, A. David, O. Davignon, A. De Roeck, M. Deile,

M. Dobson, M. Dünser, N. Dupont, A. Elliott-Peisert, N. Emriskova, F. Fallavollita ${ }^{48}$,

D. Fasanella, S. Fiorendi, G. Franzoni, J. Fulcher, W. Funk, S. Giani, D. Gigi, K. Gill,

F. Glege, L. Gouskos, M. Gruchala, M. Guilbaud, D. Gulhan, J. Hegeman, C. Heidegger,

Y. Iiyama, V. Innocente, T. James, P. Janot, O. Karacheban ${ }^{20}$, J. Kaspar, J. Kieseler,

M. Krammer ${ }^{1}$, N. Kratochwil, C. Lange, P. Lecoq, C. Lourenço, L. Malgeri, M. Mannelli,

A. Massironi, F. Meijers, S. Mersi, E. Meschi, F. Moortgat, M. Mulders, J. Ngadiuba,

J. Niedziela, S. Nourbakhsh, S. Orfanelli, L. Orsini, F. Pantaleo ${ }^{17}$, L. Pape, E. Perez,

M. Peruzzi, A. Petrilli, G. Petrucciani, A. Pfeiffer, M. Pierini, F.M. Pitters, D. Rabady,

A. Racz, M. Rieger, M. Rovere, H. Sakulin, J. Salfeld-Nebgen, C. Schäfer, C. Schwick, M. Selvaggi, A. Sharma, P. Silva, W. Snoeys, P. Sphicas ${ }^{49}$, J. Steggemann, S. Summers, V.R. Tavolaro, D. Treille, A. Tsirou, G.P. Van Onsem, A. Vartak, M. Verzetti, W.D. Zeuner

\section{Paul Scherrer Institut, Villigen, Switzerland}

L. Caminada ${ }^{50}$, K. Deiters, W. Erdmann, R. Horisberger, Q. Ingram, H.C. Kaestli, D. Kotlinski, U. Langenegger, T. Rohe, S.A. Wiederkehr

ETH Zurich - Institute for Particle Physics and Astrophysics (IPA), Zurich, Switzerland

M. Backhaus, P. Berger, N. Chernyavskaya, G. Dissertori, M. Dittmar, M. Donegà, C. Dorfer, T.A. Gómez Espinosa, C. Grab, D. Hits, W. Lustermann, R.A. Manzoni, M.T. Meinhard, F. Micheli, P. Musella, F. Nessi-Tedaldi, F. Pauss, G. Perrin, L. Perrozzi, S. Pigazzini, M.G. Ratti, M. Reichmann, C. Reissel, T. Reitenspiess, B. Ristic, D. Ruini, D.A. Sanz Becerra, M. Schönenberger, L. Shchutska, M.L. Vesterbacka Olsson, R. Wallny, D.H. Zhu

\section{Universität Zürich, Zurich, Switzerland}

T.K. Aarrestad, C. Amsler ${ }^{51}$, C. Botta, D. Brzhechko, M.F. Canelli, A. De Cosa, R. Del Burgo, B. Kilminster, S. Leontsinis, V.M. Mikuni, I. Neutelings, G. Rauco, P. Robmann, K. Schweiger, C. Seitz, Y. Takahashi, S. Wertz, A. Zucchetta

\section{National Central University, Chung-Li, Taiwan}

T.H. Doan, C.M. Kuo, W. Lin, A. Roy, S.S. Yu

\section{National Taiwan University (NTU), Taipei, Taiwan}

P. Chang, Y. Chao, K.F. Chen, P.H. Chen, W.-S. Hou, Y.y. Li, R.-S. Lu, E. Paganis, A. Psallidas, A. Steen

Chulalongkorn University, Faculty of Science, Department of Physics, Bangkok, Thailand

B. Asavapibhop, C. Asawatangtrakuldee, N. Srimanobhas, N. Suwonjandee 
Çukurova University, Physics Department, Science and Art Faculty, Adana, Turkey

A. Bat, F. Boran, A. Celik ${ }^{52}$, S. Damarseckin ${ }^{53}$, Z.S. Demiroglu, F. Dolek, C. Dozen ${ }^{54}$, I. Dumanoglu, G. Gokbulut, EmineGurpinar Guler ${ }^{55}$, Y. Guler, I. Hos ${ }^{56}$, C. Isik, E.E. Kangal ${ }^{57}$, O. Kara, A. Kayis Topaksu, U. Kiminsu, G. Onengut, K. Ozdemir ${ }^{58}$, S. Ozturk ${ }^{59}$, A.E. Simsek, U.G. Tok, S. Turkcapar, I.S. Zorbakir, C. Zorbilmez

Middle East Technical University, Physics Department, Ankara, Turkey B. Isildak ${ }^{60}$, G. Karapinar ${ }^{61}$, M. Yalvac

Bogazici University, Istanbul, Turkey

I.O. Atakisi, E. Gülmez, M. Kaya ${ }^{62}$, O. Kaya ${ }^{63}$, Ö. Özçelik, S. Tekten, E.A. Yetkin ${ }^{64}$

Istanbul Technical University, Istanbul, Turkey

A. Cakir, K. Cankocak, Y. Komurcu, S. Sen ${ }^{65}$

Istanbul University, Istanbul, Turkey

S. Cerci ${ }^{66}$, B. Kaynak, S. Ozkorucuklu, D. Sunar Cerci ${ }^{66}$

Institute for Scintillation Materials of National Academy of Science of Ukraine, Kharkov, Ukraine

B. Grynyov

National Scientific Center, Kharkov Institute of Physics and Technology, Kharkov, Ukraine

L. Levchuk

University of Bristol, Bristol, United Kingdom

E. Bhal, S. Bologna, J.J. Brooke, D. Burns ${ }^{67}$, E. Clement, D. Cussans, H. Flacher, J. Goldstein, G.P. Heath, H.F. Heath, L. Kreczko, B. Krikler, S. Paramesvaran, B. Penning, T. Sakuma, S. Seif El Nasr-Storey, V.J. Smith, J. Taylor, A. Titterton

Rutherford Appleton Laboratory, Didcot, United Kingdom

K.W. Bell, A. Belyaev ${ }^{68}$, C. Brew, R.M. Brown, D.J.A. Cockerill, J.A. Coughlan, K. Harder, S. Harper, J. Linacre, K. Manolopoulos, D.M. Newbold, E. Olaiya, D. Petyt, T. Reis, T. Schuh, C.H. Shepherd-Themistocleous, A. Thea, I.R. Tomalin, T. Williams

Imperial College, London, United Kingdom

R. Bainbridge, P. Bloch, J. Borg, S. Breeze, O. Buchmuller, A. Bundock, GurpreetSingh CHAHAL ${ }^{69}$, D. Colling, P. Dauncey, G. Davies, M. Della Negra, R. Di Maria, P. Everaerts, G. Hall, G. Iles, M. Komm, L. Lyons, A.-M. Magnan, S. Malik, A. Martelli, V. Milosevic, A. Morton, J. Nash ${ }^{70}$, V. Palladino, M. Pesaresi, D.M. Raymond, A. Richards, A. Rose, E. Scott, C. Seez, A. Shtipliyski, M. Stoye, T. Strebler, A. Tapper, K. Uchida, T. Virdee ${ }^{17}$, N. Wardle, D. Winterbottom, A.G. Zecchinelli, S.C. Zenz

Brunel University, Uxbridge, United Kingdom

J.E. Cole, P.R. Hobson, A. Khan, P. Kyberd, C.K. Mackay, I.D. Reid, L. Teodorescu, S. Zahid 
Baylor University, Waco, U.S.A.

K. Call, B. Caraway, J. Dittmann, K. Hatakeyama, C. Madrid, B. McMaster, N. Pastika, C. Smith

Catholic University of America, Washington, DC, U.S.A.

R. Bartek, A. Dominguez, R. Uniyal, A.M. Vargas Hernandez

The University of Alabama, Tuscaloosa, U.S.A.

A. Buccilli, S.I. Cooper, C. Henderson, P. Rumerio, C. West

Boston University, Boston, U.S.A.

A. Albert, D. Arcaro, Z. Demiragli, D. Gastler, C. Richardson, J. Rohlf, D. Sperka, I. Suarez, L. Sulak, D. Zou

Brown University, Providence, U.S.A.

G. Benelli, B. Burkle, X. Coubez ${ }^{18}$, D. Cutts, Y.t. Duh, M. Hadley, U. Heintz, J.M. Hogan ${ }^{71}$, K.H.M. Kwok, E. Laird, G. Landsberg, K.T. Lau, J. Lee, M. Narain, S. Sagir ${ }^{72}$, R. Syarif, E. Usai, W.Y. Wong, D. Yu, W. Zhang

University of California, Davis, Davis, U.S.A.

R. Band, C. Brainerd, R. Breedon, M. Calderon De La Barca Sanchez, M. Chertok, J. Conway, R. Conway, P.T. Cox, R. Erbacher, C. Flores, G. Funk, F. Jensen, W. Ko ${ }^{\dagger}$, O. Kukral, R. Lander, M. Mulhearn, D. Pellett, J. Pilot, M. Shi, D. Taylor, K. Tos, M. Tripathi, Z. Wang, F. Zhang

University of California, Los Angeles, U.S.A.

M. Bachtis, C. Bravo, R. Cousins, A. Dasgupta, A. Florent, J. Hauser, M. Ignatenko, N. Mccoll, W.A. Nash, S. Regnard, D. Saltzberg, C. Schnaible, B. Stone, V. Valuev

University of California, Riverside, Riverside, U.S.A.

K. Burt, Y. Chen, R. Clare, J.W. Gary, S.M.A. Ghiasi Shirazi, G. Hanson, G. Karapostoli, O.R. Long, M. Olmedo Negrete, M.I. Paneva, W. Si, L. Wang, S. Wimpenny, B.R. Yates, Y. Zhang

University of California, San Diego, La Jolla, U.S.A.

J.G. Branson, P. Chang, S. Cittolin, S. Cooperstein, N. Deelen, M. Derdzinski, R. Gerosa, D. Gilbert, B. Hashemi, D. Klein, V. Krutelyov, J. Letts, M. Masciovecchio, S. May, S. Padhi, M. Pieri, V. Sharma, M. Tadel, F. Würthwein, A. Yagil, G. Zevi Della Porta

University of California, Santa Barbara - Department of Physics, Santa Barbara, U.S.A.

N. Amin, R. Bhandari, C. Campagnari, M. Citron, V. Dutta, M. Franco Sevilla, J. Incandela, B. Marsh, H. Mei, A. Ovcharova, H. Qu, J. Richman, U. Sarica, D. Stuart, S. Wang

California Institute of Technology, Pasadena, U.S.A.

D. Anderson, A. Bornheim, O. Cerri, I. Dutta, J.M. Lawhorn, N. Lu, J. Mao, H.B. Newman, T.Q. Nguyen, J. Pata, M. Spiropulu, J.R. Vlimant, S. Xie, Z. Zhang, R.Y. Zhu 
Carnegie Mellon University, Pittsburgh, U.S.A.

M.B. Andrews, T. Ferguson, T. Mudholkar, M. Paulini, M. Sun, I. Vorobiev, M. Weinberg University of Colorado Boulder, Boulder, U.S.A.

J.P. Cumalat, W.T. Ford, E. MacDonald, T. Mulholland, R. Patel, A. Perloff, K. Stenson, K.A. Ulmer, S.R. Wagner

Cornell University, Ithaca, U.S.A.

J. Alexander, Y. Cheng, J. Chu, A. Datta, A. Frankenthal, K. Mcdermott, J.R. Patterson, D. Quach, A. Ryd, S.M. Tan, Z. Tao, J. Thom, P. Wittich, M. Zientek

Fermi National Accelerator Laboratory, Batavia, U.S.A.

S. Abdullin, M. Albrow, M. Alyari, G. Apollinari, A. Apresyan, A. Apyan, S. Banerjee, L.A.T. Bauerdick, A. Beretvas, D. Berry, J. Berryhill, P.C. Bhat, K. Burkett, J.N. Butler, A. Canepa, G.B. Cerati, H.W.K. Cheung, F. Chlebana, M. Cremonesi, J. Duarte, V.D. Elvira, J. Freeman, Z. Gecse, E. Gottschalk, L. Gray, D. Green, S. Grünendahl, O. Gutsche, J. Hanlon, R.M. Harris, S. Hasegawa, R. Heller, J. Hirschauer, B. Jayatilaka, S. Jindariani, M. Johnson, U. Joshi, T. Klijnsma, B. Klima, M.J. Kortelainen, B. Kreis, S. Lammel, J. Lewis, D. Lincoln, R. Lipton, M. Liu, T. Liu, J. Lykken, K. Maeshima, J.M. Marraffino, D. Mason, P. McBride, P. Merkel, S. Mrenna, S. Nahn, V. O’Dell, V. Papadimitriou, K. Pedro, C. Pena, G. Rakness, F. Ravera, A. Reinsvold Hall, L. Ristori, B. Schneider, E. Sexton-Kennedy, N. Smith, A. Soha, W.J. Spalding, L. Spiegel, S. Stoynev, J. Strait, N. Strobbe, L. Taylor, S. Tkaczyk, N.V. Tran, L. Uplegger, E.W. Vaandering, C. Vernieri, R. Vidal, M. Wang, H.A. Weber

University of Florida, Gainesville, U.S.A.

D. Acosta, P. Avery, D. Bourilkov, A. Brinkerhoff, L. Cadamuro, V. Cherepanov, F. Errico, R.D. Field, S.V. Gleyzer, D. Guerrero, B.M. Joshi, M. Kim, J. Konigsberg, A. Korytov, K.H. Lo, K. Matchev, N. Menendez, G. Mitselmakher, D. Rosenzweig, K. Shi, J. Wang, S. Wang, X. Zuo

Florida International University, Miami, U.S.A.

Y.R. Joshi

Florida State University, Tallahassee, U.S.A.

T. Adams, A. Askew, S. Hagopian, V. Hagopian, K.F. Johnson, R. Khurana, T. Kolberg, G. Martinez, T. Perry, H. Prosper, C. Schiber, R. Yohay, J. Zhang

Florida Institute of Technology, Melbourne, U.S.A.

M.M. Baarmand, M. Hohlmann, D. Noonan, M. Rahmani, M. Saunders, F. Yumiceva

University of Illinois at Chicago (UIC), Chicago, U.S.A.

M.R. Adams, L. Apanasevich, R.R. Betts, R. Cavanaugh, X. Chen, S. Dittmer, O. Evdokimov, C.E. Gerber, D.A. Hangal, D.J. Hofman, C. Mills, T. Roy, M.B. Tonjes, N. Varelas, J. Viinikainen, H. Wang, X. Wang, Z. Wu 
The University of Iowa, Iowa City, U.S.A.

M. Alhusseini, B. Bilki ${ }^{55}$, K. Dilsiz ${ }^{73}$, S. Durgut, R.P. Gandrajula, M. Haytmyradov, V. Khristenko, O.K. Köseyan, J.-P. Merlo, A. Mestvirishvilii ${ }^{74}$, A. Moeller, J. Nachtman, H. Ogul ${ }^{75}$, Y. Onel, F. Ozok ${ }^{76}$, A. Penzo, C. Snyder, E. Tiras, J. Wetzel

Johns Hopkins University, Baltimore, U.S.A.

B. Blumenfeld, A. Cocoros, N. Eminizer, A.V. Gritsan, W.T. Hung, S. Kyriacou, P. Maksimovic, J. Roskes, M. Swartz

The University of Kansas, Lawrence, U.S.A.

C. Baldenegro Barrera, P. Baringer, A. Bean, S. Boren, J. Bowen, A. Bylinkin, T. Isidori, S. Khalil, J. King, G. Krintiras, A. Kropivnitskaya, C. Lindsey, D. Majumder, W. Mcbrayer, N. Minafra, M. Murray, C. Rogan, C. Royon, S. Sanders, E. Schmitz, J.D. Tapia Takaki, Q. Wang, J. Williams, G. Wilson

Kansas State University, Manhattan, U.S.A.

S. Duric, A. Ivanov, K. Kaadze, D. Kim, Y. Maravin, D.R. Mendis, T. Mitchell, A. Modak, A. Mohammadi

Lawrence Livermore National Laboratory, Livermore, U.S.A.

F. Rebassoo, D. Wright

University of Maryland, College Park, U.S.A.

A. Baden, O. Baron, A. Belloni, S.C. Eno, Y. Feng, N.J. Hadley, S. Jabeen, G.Y. Jeng, R.G. Kellogg, A.C. Mignerey, S. Nabili, F. Ricci-Tam, M. Seidel, Y.H. Shin, A. Skuja, S.C. Tonwar, K. Wong

Massachusetts Institute of Technology, Cambridge, U.S.A.

D. Abercrombie, B. Allen, A. Baty, R. Bi, S. Brandt, W. Busza, I.A. Cali, M. D'Alfonso, G. Gomez Ceballos, M. Goncharov, P. Harris, D. Hsu, M. Hu, M. Klute, D. Kovalskyi, Y.-J. Lee, P.D. Luckey, B. Maier, A.C. Marini, C. Mcginn, C. Mironov, S. Narayanan, X. Niu, C. Paus, D. Rankin, C. Roland, G. Roland, Z. Shi, G.S.F. Stephans, K. Sumorok, K. Tatar, D. Velicanu, J. Wang, T.W. Wang, B. Wyslouch

University of Minnesota, Minneapolis, U.S.A.

R.M. Chatterjee, A. Evans, S. Guts ${ }^{\dagger}$, P. Hansen, J. Hiltbrand, Sh. Jain, Y. Kubota, Z. Lesko, J. Mans, M. Revering, R. Rusack, R. Saradhy, N. Schroeder, M.A. Wadud

University of Mississippi, Oxford, U.S.A.

J.G. Acosta, S. Oliveros

University of Nebraska-Lincoln, Lincoln, U.S.A.

K. Bloom, S. Chauhan, D.R. Claes, C. Fangmeier, L. Finco, F. Golf, R. Kamalieddin, I. Kravchenko, J.E. Siado, G.R. Snow ${ }^{\dagger}$, B. Stieger, W. Tabb

State University of New York at Buffalo, Buffalo, U.S.A.

G. Agarwal, C. Harrington, I. Iashvili, A. Kharchilava, C. McLean, D. Nguyen, A. Parker, J. Pekkanen, S. Rappoccio, B. Roozbahani 
Northeastern University, Boston, U.S.A.

G. Alverson, E. Barberis, C. Freer, Y. Haddad, A. Hortiangtham, G. Madigan, B. Marzocchi, D.M. Morse, T. Orimoto, L. Skinnari, A. Tishelman-Charny, T. Wamorkar, B. Wang, A. Wisecarver, D. Wood

Northwestern University, Evanston, U.S.A.

S. Bhattacharya, J. Bueghly, A. Gilbert, T. Gunter, K.A. Hahn, N. Odell, M.H. Schmitt, K. Sung, M. Trovato, M. Velasco

University of Notre Dame, Notre Dame, U.S.A.

R. Bucci, N. Dev, R. Goldouzian, M. Hildreth, K. Hurtado Anampa, C. Jessop, D.J. Karmgard, K. Lannon, W. Li, N. Loukas, N. Marinelli, I. Mcalister, F. Meng, Y. Musienko ${ }^{38}$, R. Ruchti, P. Siddireddy, G. Smith, S. Taroni, M. Wayne, A. Wightman, M. Wolf, A. Woodard

\section{The Ohio State University, Columbus, U.S.A.}

J. Alimena, B. Bylsma, L.S. Durkin, B. Francis, C. Hill, W. Ji, A. Lefeld, T.Y. Ling, B.L. Winer

Princeton University, Princeton, U.S.A.

G. Dezoort, P. Elmer, J. Hardenbrook, N. Haubrich, S. Higginbotham, A. Kalogeropoulos, S. Kwan, D. Lange, M.T. Lucchini, J. Luo, D. Marlow, K. Mei, I. Ojalvo, J. Olsen, C. Palmer, P. Piroué, D. Stickland, C. Tully

University of Puerto Rico, Mayaguez, U.S.A.

S. Malik, S. Norberg

Purdue University, West Lafayette, U.S.A.

A. Barker, V.E. Barnes, S. Das, L. Gutay, M. Jones, A.W. Jung, A. Khatiwada, B. Mahakud, D.H. Miller, G. Negro, N. Neumeister, C.C. Peng, S. Piperov, H. Qiu, J.F. Schulte, N. Trevisani, F. Wang, R. Xiao, W. Xie

Purdue University Northwest, Hammond, U.S.A.

T. Cheng, J. Dolen, N. Parashar

Rice University, Houston, U.S.A.

U. Behrens, S. Dildick, K.M. Ecklund, S. Freed, F.J.M. Geurts, M. Kilpatrick, Arun Kumar, W. Li, B.P. Padley, R. Redjimi, J. Roberts, J. Rorie, W. Shi, A.G. Stahl Leiton, Z. Tu, A. Zhang

University of Rochester, Rochester, U.S.A.

A. Bodek, P. de Barbaro, R. Demina, J.L. Dulemba, C. Fallon, T. Ferbel, M. Galanti, A. Garcia-Bellido, O. Hindrichs, A. Khukhunaishvili, E. Ranken, R. Taus

Rutgers, The State University of New Jersey, Piscataway, U.S.A.

B. Chiarito, J.P. Chou, A. Gandrakota, Y. Gershtein, E. Halkiadakis, A. Hart, M. Heindl, E. Hughes, S. Kaplan, I. Laflotte, A. Lath, R. Montalvo, K. Nash, M. Osherson, H. Saka, S. Salur, S. Schnetzer, S. Somalwar, R. Stone, S. Thomas 
University of Tennessee, Knoxville, U.S.A.

H. Acharya, A.G. Delannoy, S. Spanier

Texas A\&M University, College Station, U.S.A.

O. Bouhali ${ }^{77}$, M. Dalchenko, M. De Mattia, A. Delgado, R. Eusebi, J. Gilmore, T. Huang, T. Kamon ${ }^{78}$, H. Kim, S. Luo, S. Malhotra, D. Marley, R. Mueller, D. Overton, L. Perniè,

D. Rathjens, A. Safonov

Texas Tech University, Lubbock, U.S.A.

N. Akchurin, J. Damgov, F. De Guio, V. Hegde, S. Kunori, K. Lamichhane, S.W. Lee, T. Mengke, S. Muthumuni, T. Peltola, S. Undleeb, I. Volobouev, Z. Wang, A. Whitbeck

Vanderbilt University, Nashville, U.S.A.

S. Greene, A. Gurrola, R. Janjam, W. Johns, C. Maguire, A. Melo, H. Ni, K. Padeken, F. Romeo, P. Sheldon, S. Tuo, J. Velkovska, M. Verweij

University of Virginia, Charlottesville, U.S.A.

M.W. Arenton, P. Barria, B. Cox, G. Cummings, J. Hakala, R. Hirosky, M. Joyce, A. Ledovskoy, C. Neu, B. Tannenwald, Y. Wang, E. Wolfe, F. Xia

Wayne State University, Detroit, U.S.A.

R. Harr, P.E. Karchin, N. Poudyal, J. Sturdy, P. Thapa

University of Wisconsin - Madison, Madison, WI, U.S.A.

T. Bose, J. Buchanan, C. Caillol, D. Carlsmith, S. Dasu, I. De Bruyn, L. Dodd, C. Galloni, H. He, M. Herndon, A. Hervé, U. Hussain, A. Lanaro, A. Loeliger, K. Long, R. Loveless, J. Madhusudanan Sreekala, D. Pinna, T. Ruggles, A. Savin, V. Sharma, W.H. Smith, D. Teague, S. Trembath-reichert

$\dagger$ : Deceased

1: Also at Vienna University of Technology, Vienna, Austria

2: Also at IRFU, CEA, Université Paris-Saclay, Gif-sur-Yvette, France

3: Also at Universidade Estadual de Campinas, Campinas, Brazil

4: Also at Federal University of Rio Grande do Sul, Porto Alegre, Brazil

5: Also at UFMS, Nova Andradina, Brazil

6: Also at Universidade Federal de Pelotas, Pelotas, Brazil

7: Also at Université Libre de Bruxelles, Bruxelles, Belgium

8: Also at University of Chinese Academy of Sciences, Beijing, China

9: Also at Institute for Theoretical and Experimental Physics named by A.I. Alikhanov of NRC 'Kurchatov Institute', Moscow, Russia

10: Also at Joint Institute for Nuclear Research, Dubna, Russia

11: Also at Cairo University, Cairo, Egypt

12: Now at British University in Egypt, Cairo, Egypt

13: Also at Purdue University, West Lafayette, U.S.A.

14: Also at Université de Haute Alsace, Mulhouse, France

15: Also at Tbilisi State University, Tbilisi, Georgia

16: Also at Erzincan Binali Yildirim University, Erzincan, Turkey

17: Also at CERN, European Organization for Nuclear Research, Geneva, Switzerland 
18: Also at RWTH Aachen University, III. Physikalisches Institut A, Aachen, Germany

19: Also at University of Hamburg, Hamburg, Germany

20: Also at Brandenburg University of Technology, Cottbus, Germany

21: Also at Institute of Physics, University of Debrecen, Debrecen, Hungary, Debrecen, Hungary

22: Also at Institute of Nuclear Research ATOMKI, Debrecen, Hungary

23: Also at MTA-ELTE Lendület CMS Particle and Nuclear Physics Group, Eötvös Loránd University, Budapest, Hungary, Budapest, Hungary

24: Also at IIT Bhubaneswar, Bhubaneswar, India, Bhubaneswar, India

25: Also at Institute of Physics, Bhubaneswar, India

26: Also at Shoolini University, Solan, India

27: Also at University of Hyderabad, Hyderabad, India

28: Also at University of Visva-Bharati, Santiniketan, India

29: Also at Isfahan University of Technology, Isfahan, Iran

30: Now at INFN Sezione di Bari ${ }^{a}$, Università di Bari ${ }^{b}$, Politecnico di Bari ${ }^{c}$, Bari, Italy

31: Also at Italian National Agency for New Technologies, Energy and Sustainable Economic Development, Bologna, Italy

32: Also at Centro Siciliano di Fisica Nucleare e di Struttura Della Materia, Catania, Italy

33: Also at Scuola Normale e Sezione dell'INFN, Pisa, Italy

34: Also at Riga Technical University, Riga, Latvia, Riga, Latvia

35: Also at Malaysian Nuclear Agency, MOSTI, Kajang, Malaysia

36: Also at Consejo Nacional de Ciencia y Tecnología, Mexico City, Mexico

37: Also at Warsaw University of Technology, Institute of Electronic Systems, Warsaw, Poland

38: Also at Institute for Nuclear Research, Moscow, Russia

39: Now at National Research Nuclear University 'Moscow Engineering Physics Institute' (MEPhI), Moscow, Russia

40: Also at St. Petersburg State Polytechnical University, St. Petersburg, Russia

41: Also at University of Florida, Gainesville, U.S.A.

42: Also at Imperial College, London, United Kingdom

43: Also at P.N. Lebedev Physical Institute, Moscow, Russia

44: Also at California Institute of Technology, Pasadena, U.S.A.

45: Also at Budker Institute of Nuclear Physics, Novosibirsk, Russia

46: Also at Faculty of Physics, University of Belgrade, Belgrade, Serbia

47: Also at Università degli Studi di Siena, Siena, Italy

48: Also at INFN Sezione di Pavia ${ }^{a}$, Università di Pavia ${ }^{b}$, Pavia, Italy, Pavia, Italy

49: Also at National and Kapodistrian University of Athens, Athens, Greece

50: Also at Universität Zürich, Zurich, Switzerland

51: Also at Stefan Meyer Institute for Subatomic Physics, Vienna, Austria, Vienna, Austria

52: Also at Burdur Mehmet Akif Ersoy University, BURDUR, Turkey

53: Also at Şırnak University, Sirnak, Turkey

54: Also at Department of Physics, Tsinghua University, Beijing, China, Beijing, China

55: Also at Beykent University, Istanbul, Turkey, Istanbul, Turkey

56: Also at Istanbul Aydin University, Application and Research Center for Advanced Studies (App. \& Res. Cent. for Advanced Studies), Istanbul, Turkey

57: Also at Mersin University, Mersin, Turkey

58: Also at Piri Reis University, Istanbul, Turkey

59: Also at Gaziosmanpasa University, Tokat, Turkey

60: Also at Ozyegin University, Istanbul, Turkey

61: Also at Izmir Institute of Technology, Izmir, Turkey 
62: Also at Marmara University, Istanbul, Turkey

63: Also at Kafkas University, Kars, Turkey

64: Also at Istanbul Bilgi University, Istanbul, Turkey

65: Also at Hacettepe University, Ankara, Turkey

66: Also at Adiyaman University, Adiyaman, Turkey

67: Also at Vrije Universiteit Brussel, Brussel, Belgium

68: Also at School of Physics and Astronomy, University of Southampton, Southampton, United Kingdom

69: Also at IPPP Durham University, Durham, United Kingdom

70: Also at Monash University, Faculty of Science, Clayton, Australia

71: Also at Bethel University, St. Paul, Minneapolis, U.S.A., St. Paul, U.S.A.

72: Also at Karamanoğlu Mehmetbey University, Karaman, Turkey

73: Also at Bingol University, Bingol, Turkey

74: Also at Georgian Technical University, Tbilisi, Georgia

75: Also at Sinop University, Sinop, Turkey

76: Also at Mimar Sinan University, Istanbul, Istanbul, Turkey

77: Also at Texas A\&M University at Qatar, Doha, Qatar

78: Also at Kyungpook National University, Daegu, Korea, Daegu, Korea 